\title{
Off-shell top quarks with one jet at the LHC: a comprehensive analysis at NLO QCD
}

\author{
G. Bevilacqua, ${ }^{a}$ H.B. Hartanto, ${ }^{b}$ M. Kraus ${ }^{b}$ and M. Worek ${ }^{b}$ \\ ${ }^{a}$ MTA-DE Particle Physics Research Group, University of Debrecen, \\ H-4010 Debrecen, Hungary \\ ${ }^{b}$ Institute for Theoretical Particle Physics and Cosmology, RWTH Aachen University, \\ D-52056 Aachen, Germany \\ E-mail: giuseppe.bevilacqua@science.unideb.hu, \\ hartanto@physik.rwth-aachen.de, kraus@physik.rwth-aachen.de, \\ worek@physik.rwth-aachen.de
}

ABSTRACT: We present a comprehensive study of the production of top quark pairs in association with one hard jet in the di-lepton decay channel at the LHC. Our predictions, accurate at NLO in QCD, focus on the LHC Run II with a center-of-mass energy of $13 \mathrm{TeV}$. All resonant and non-resonant contributions at the perturbative order $\mathcal{O}\left(\alpha_{s}^{4} \alpha^{4}\right)$ are taken into account, including irreducible backgrounds to $t \bar{t} j$ production, interferences and off-shell effects of the top quark and the $W$ gauge boson. We extensively investigate the dependence of our results upon variation of renormalisation and factorisation scales and parton distribution functions in the quest for an accurate estimate of the theoretical uncertainties. Additionally, we explore a few possibilities for a dynamical scale choice with the goal of stabilizing the perturbative convergence of the differential cross sections far away from the $t \bar{t}$ threshold. Results presented here are particularly relevant for searches of new physics as well as for precise measurements of the top-quark fiducial cross sections and top-quark properties at the LHC.

KEYwORDS: NLO Computations, QCD Phenomenology

ARXiv EPrint: 1609.01659 


\section{Contents}

1 Introduction 1

2 Details of the calculations 4

3 Results for the LHC Run II energy of $13 \mathrm{TeV} \quad 10$

$\begin{array}{ll}3.1 \text { Numerical setup } & 10\end{array}$

$\begin{array}{lll}3.2 & \text { Integrated cross sections with theoretical uncertainties } & 13\end{array}$

$\begin{array}{lll}3.3 & \text { Differential distributions } & 19\end{array}$

3.4 Theoretical uncertainties for differential cross sections 34

4 Conclusions $\quad 40$

\section{Introduction}

The large top-quark pair production cross section at the LHC, a $p p$ collider, makes it a unique laboratory for studying the behaviour of QCD at the highest accessible energy to date. Besides the determination of the top-quark mass $\left(m_{t}\right)$ and the strong coupling constant $\left(\alpha_{s}\right)$ key measurements include fiducial cross sections, various infra-red safe differential distributions, spin correlations, inclusive top-quark charge asymmetry as well as leptonic charge asymmetry together with top-quark couplings to gauge bosons and the Standard Model (SM) Higgs boson. In addition, work on constraining parton distribution functions mainly for the dominant gluon-gluon production channel with the help of the total cross section and various differential distributions is ongoing. The decays of top quarks to charged leptons, neutrinos and b-quarks make this process a primary source of background in many searches for new physics. Therefore, an accurate modelling of topquark events forms an important part of the LHC physics programme. At LHC energies, however, a large fraction of top-quark pairs is accompanied by additional hard jets. To estimate the size of the $t \bar{t} j$ contribution in the inclusive $t \bar{t}$ sample we show in table 1 the cross section for the on-shell $p p \rightarrow t \bar{t} j$ production at NLO in QCD with various $p_{T, j}$ cuts on the hard jet. Also shown is its ratio to the inclusive $p p \rightarrow t \bar{t}$ production at the same level of accuracy. Results are given for the LHC Run II energy of $13 \mathrm{TeV}$, the top-quark mass of $m_{t}=173.2 \mathrm{GeV}$ and for $\mu=\mu_{R}=\mu_{F}=m_{t}$. For parton distribution functions (PDFs), the CT14nlo set has been employed. We can observe that, for example, by requiring a minimal transverse momentum of $40 \mathrm{GeV}$ for the additional jets will result in a contribution from $t \bar{t} j$ events of more than $40 \%$. From an experimental point of view, jets not originating from the decay of the top quark and top antiquark, but arising from quark and gluon radiation produced in association with the $t \bar{t}$ system need to be understood very precisely since their appearance affects the reconstruction of the $t \bar{t}$ event. The additional 


\begin{tabular}{|c|c|c|}
\hline$p_{T, j} \quad[\mathrm{GeV}]$ & $\sigma_{t \bar{t} j}^{\mathrm{NLO}} \quad[\mathrm{pb}]$ & RATIO [\%] \\
\hline 40 & $296.97 \pm 0.29$ & 41 \\
\hline 60 & $207.88 \pm 0.19$ & 29 \\
\hline 80 & $152.89 \pm 0.13$ & 21 \\
\hline 100 & $115.60 \pm 0.14$ & 16 \\
\hline 120 & $89.05 \pm 0.10$ & 12 \\
\hline
\end{tabular}

Table 1. The NLO cross section for the on-shell $p p \rightarrow t \bar{t} j+X$ production with various values for $p_{T, j}$ cut on the hardest jet. Also shown is its ratios to the NLO cross section for the on-shell $p p \rightarrow t \bar{t}+X$ production. Results are obtained for the LHC Run II energy of $13 \mathrm{TeV}$, the top-quark mass of $m_{t}=173.2 \mathrm{GeV}, \mu_{R}=\mu_{F}=m_{t}$ and for the CT14nlo PDF set.

jet activity can be used to examine the underlying production and decay mechanisms even further and to design new methods for a sizeable reduction of QCD backgrounds [1-3]. Because of its large production rate, the $p p \rightarrow t \bar{t} j$ process is a sizeable background process for SM studies or searches for new physics that involve a production of $W^{+} W^{-}$gauge boson pairs in association with jets [4-7]. The most prominent example is SM Higgs boson production in vector boson fusion, where $t \bar{t} j$ production is the dominant background process $[8,9]$. Another example is the production of top-quark flavour violating resonances that can be singly produced in association with the top quark at the LHC [10]. Searches for new heavy resonances, a color singlet $W^{\prime}$ or a colour triplet $\phi^{a}$, produced in association with the top quark have been performed by both ATLAS and CMS collaborations. Limits on the mass and the coupling of $W^{\prime}$ and $\phi^{a}$ have been set by analyzing the $t+q$ and the $\bar{t}+q$ invariant mass spectrum in $t \bar{t} j$ candidate events [11, 12]. A search for new physics can be also performed by looking for effects on the top-quark dipole moments, i.e. chromo-electric dipole and chromo-magnetic dipole moments, which can be parametrised by adding an effective term to the top-quark-gluon gauge coupling [13-15]. Anomalous $t \bar{t} g$ couplings would lead to a significant modification of the $t \bar{t}$ spin correlation in $t \bar{t}$ and $t \bar{t} j$ systems that might be visualised in the normalised cross sections as a function of the difference in azimuthal angle between the two charged leptons, $|\Delta \phi(\ell \ell)|$, in the dilepton decay mode $[16,17]$. Additionally, $t \bar{t} j$ production can be employed in the top-quark mass extraction by studying normalised differential cross sections as a function of the inverse invariant mass of the $t \bar{t} j$ system [18]. The method has already been successfully used by experimental groups at the LHC $[19,20]$. Both the ATLAS and CMS collaborations are carefully examining $p p \rightarrow t \bar{t}+$ jets production. The studies performed at the LHC include measurements of jet activity in top-quark events, measurements of $t \bar{t}$ production with a veto on additional central jet activity and measurements of heavy flavor composition of $t \bar{t}$ events [21-27]. For example, the ATLAS experiment has measured using $4.6 \mathrm{fb}^{-1}$ of data at $\sqrt{s}=7 \mathrm{TeV}$ the fiducial $t \bar{t}$ cross section as a function of the light jet multiplicity for up to eight jets with jet $p_{T}$ thresholds of $25,40,60$, and $80 \mathrm{GeV}$. A precision of the order of $10 \%$ has been obtained for the $\sigma_{t \bar{t} j}$ contribution, while for the differential cross section as a function of transverse momentum of the hardest light jet, a precision between 
$10 \%$ and $16 \%$ has been reached. Similar studies have been repeated at 8 and $13 \mathrm{TeV}$ with $20.3 \mathrm{fb}^{-1}$ and $3.2 \mathrm{fb}^{-1}$ of $p p$ collision data respectively. In the former case experimental uncertainties remain the same, whereas in the latter they are quite large, of the order of $25 \%-40 \%$, due to low statistics. However, the situation will improve very soon once more data is analysed. On the other hand, a very recent CMS study [28] at $\sqrt{s}=8 \mathrm{TeV}$ with an integrated luminosity of $19.7 \mathrm{fb}^{-1}$ has shown that the total cross section for $t \bar{t}+\geq 1$ jet production can be measured with the total experimental uncertainty of the order of $7 \%$. Additional jet activity in $t \bar{t}$ events has also been investigated by analysing the so-called gap-fraction distributions. The ATLAS and CMS collaborations have vetoed events that contain an additional jet with transverse momentum above a given threshold in a central rapidity interval. The fraction of events surviving the jet veto, i.e. the gap fraction, has been presented in these studies as a function of the threshold. Owing to the rich top-quark physics program at the LHC and to the precision, which has already been achieved, it is of great importance to reduce uncertainties for the $t \bar{t} j$ process also on the theory side. In this respect, the need of precise theoretical predictions for various physical observables in the $p p \rightarrow t \bar{t} j$ production process is indisputable.

The NLO corrections to $p p \rightarrow t \bar{t} j+X$ production have first been calculated in $[29,30]$ for stable top quarks. Afterwards, LO top-quark decays in the narrow width approximation (NWA) have been included [31]. Subsequently, NLO top-quark decays in the NWA, including $t \rightarrow W b j$, have been added consistently [32]. A different approach to this process is described in [33-35], where on-shell $t \bar{t} j+X$ production at NLO QCD is matched to parton shower programs following either the POWHEG procedure [36-38] or the MC@NLO one [39]. Finally, very recently, a complete description of top-quark pair production in association with a jet in the dilepton channel has been provided at NLO in QCD [40]. In this calculation all non-resonant diagrams, interferences and off-shell effects of the top quark have been consistently taken into account together with non-resonant and off-shell effects due to the finite $W$ gauge boson width. The integrated cross section together with the scale dependence of the total cross section and a few differential cross sections for the LHC Run I centre-of-mass energy of $\sqrt{s}=8 \mathrm{TeV}$ have been studied there. In this paper we extend our previous study on the NLO QCD corrections to $p p \rightarrow e^{+} \nu_{e} \mu^{-} \bar{\nu}_{\mu} b \bar{b} j+X$ production at the LHC. We shall supplement the previous discussion with more results for the current LHC centre-of-mass energy of $\sqrt{s}=13 \mathrm{TeV}$. To be more precise, we shall present integrated and differential cross sections and estimate theoretical uncertainties as provided by the scale variation. Furthermore, we shall include dynamical scales in our study. Moreover various PDF parameterisations will be studied and in each case internal PDF uncertainties will be evaluated.

Let us note at this point, that full off-shell top-quark effects at NLO have already been considered in the literature for simpler processes, i.e. top-quark pair production and top-quark pair production in association with the SM Higgs boson [41-48].

The article is organised as follows. In the next section we describe the details of our calculation. All ingredients, methods and Monte Carlo programs, that are needed for our NLO QCD calculations, are listed and described briefly. In section 2 we additionally list all checks that have been performed to ensure the correctness of our results. Numerical 


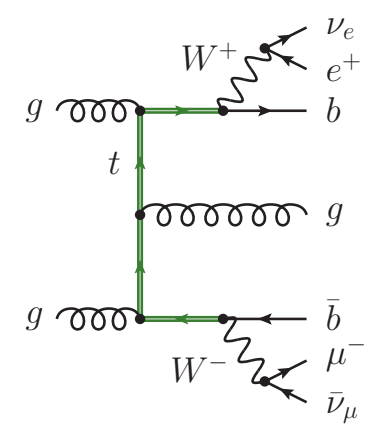

(a)

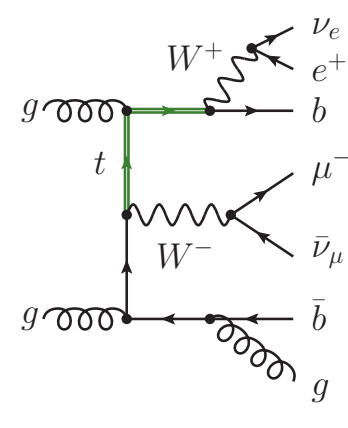

(b)

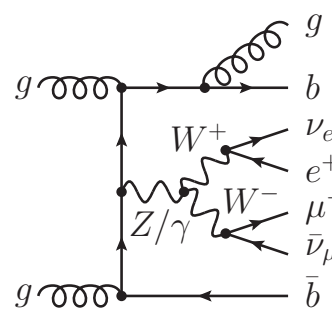

(c)

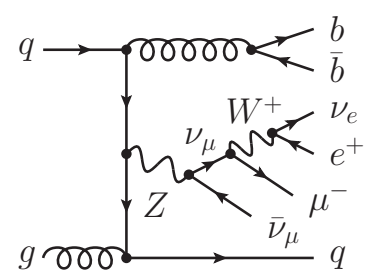

$(d)$

Figure 1. A representative set of Feynman diagrams, involving two (a), one (b) and no top-quark resonances (c), contributing to the leading order $p p \rightarrow e^{+} \nu_{e} \mu^{-} \bar{\nu}_{\mu} b \bar{b} j+X$ process at $\mathcal{O}\left(\alpha_{s}^{3} \alpha^{4}\right)$. The last diagram (d) with a single $W$ boson resonance contributes to the off-shell effects of the $W$ gauge boson.

results for the integrated and differential cross sections for various renormalisation, $\mu_{R}$, and factorisation, $\mu_{F}$, scale choices are presented in section 3. The theoretical uncertainty of the total cross section, associated with neglected higher order terms in the perturbative expansion, which are estimated by varying the renormalisation and factorisation scales independently by a factor 2, are also given there. Additionally, the theoretical uncertainty stemming from various parameterisations of PDFs are investigated in section 3 together with their internal PDFs errors. Finally, in section 4 we give our conclusions.

\section{Details of the calculations}

For the $p p \rightarrow e^{+} \nu_{e} \mu^{-} \bar{\nu}_{\mu} b \bar{b} j+X$ production process at the leading order (LO) in perturbative expansion and at $\mathcal{O}\left(\alpha_{s}^{3} \alpha^{4}\right)$, the contribution from the following partonic subprocesses need to be taken into account:

$$
\begin{aligned}
& g g \rightarrow e^{+} \nu_{e} \mu^{-} \bar{\nu}_{\mu} b \bar{b} g, \\
& g q \rightarrow e^{+} \nu_{e} \mu^{-} \bar{\nu}_{\mu} b \bar{b} q, \\
& g \bar{q} \rightarrow e^{+} \nu_{e} \mu^{-} \bar{\nu}_{\mu} b \bar{b} \bar{q}, \\
& q \bar{q} \rightarrow e^{+} \nu_{e} \mu^{-} \bar{\nu}_{\mu} b \bar{b} g,
\end{aligned}
$$

where $q=u, d, c, s$. A representative set of Feynman diagrams contributing to the process under consideration is depicted in figure 1. In total, the $g g \rightarrow e^{+} \nu_{e} \mu^{-} \bar{\nu}_{\mu} b \bar{b} g$ subprocess involves 508 tree diagrams, on the other hand the $q \bar{q} \rightarrow e^{+} \nu_{e} \mu^{-} \bar{\nu}_{\mu} b \bar{b} g$ subprocess as well as $g q \rightarrow e^{+} \nu_{e} \mu^{-} \bar{\nu}_{\mu} b \bar{b} q$ and $g \bar{q} \rightarrow e^{+} \nu_{e} \mu^{-} \bar{\nu}_{\mu} b \bar{b} \bar{q}$ subprocesses, that are all related by crossing symmetry, comprise 234 tree diagrams each. Even though we do not actually employ Feynman diagrams in our calculations, we present them as a measure of the complexity. The calculation of scattering amplitudes is performed by means of an automatic off-shell iterative algorithm [49-51], which is implemented within the HELAC-DiPOLES package [52] and the Helac-Phegas Monte Carlo program [53, 54]. The latter framework has been used 


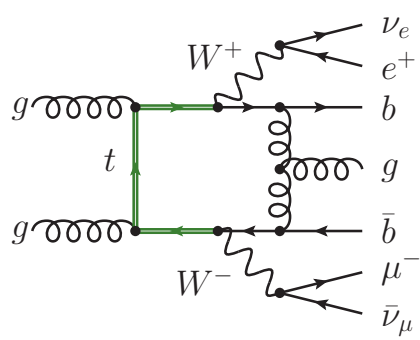

(a)

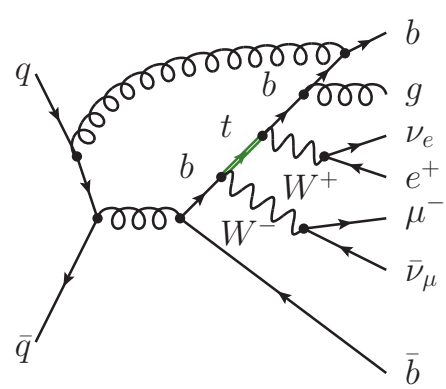

(d)

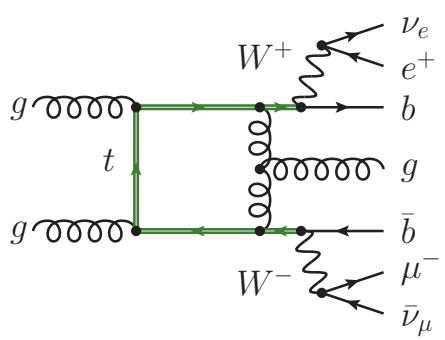

(b)

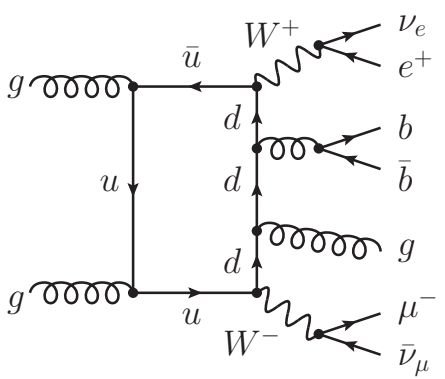

(e)

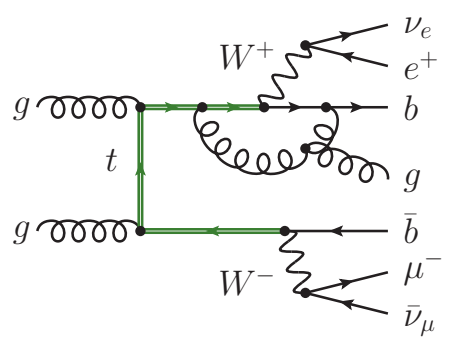

$(c)$

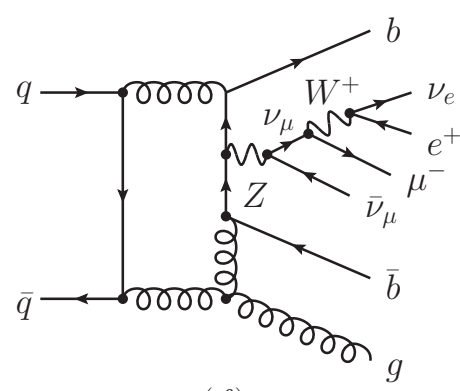

$(f)$

Figure 2. A representative set of Feynman diagrams, involving heptagons (a and d), hexagons (e and $\mathrm{f}$ ), the pentagon diagram (b) and the box diagram (c) contributing to virtual corrections to the $p p \rightarrow e^{+} \nu_{e} \mu^{-} \bar{\nu}_{\mu} b \bar{b} j+X$ process at $\mathcal{O}\left(\alpha_{s}^{4} \alpha^{4}\right)$.

to cross check our LO results. For the phase-space integration PARNI [55] and KALEU [56] have been employed.

At NLO, virtual corrections are obtained from the interference of the one-loop diagrams with the tree level amplitude. They can be classified into self-energy, vertex-, box-, pentagon-, hexagon- and heptagon-type corrections. A representative set of one-loop diagrams contributing to the process is shown in figure 2. To give an estimate of the complexity of the calculations we present the number of one-loop Feynman diagrams as obtained with QGRAF [57]. For the dominant gluon-gluon production channel 39180 one-loop diagrams have been counted. In more details, the most complex contributions comprise 120 heptagons and 1155 hexagons with tensor integrals up to rank six. Virtual corrections are evaluated in $d=4-2 \epsilon$ dimensions in the 't Hooft-Veltman version of the dimensional regularisation [58] within the Feynman gauge for gauge bosons. The singularities coming from infrared divergent pieces are canceled by the corresponding ones arising from the counterterms of the adopted subtraction scheme integrated over the phase space of the unresolved parton. The finite contributions of the loop diagrams are evaluated numerically in $d=4$ dimensions. To ensure numerical stability of our calculations we perform a few tests. Since every partonic subprocess at $\mathcal{O}\left(\alpha_{s}^{4} \alpha^{4}\right)$ has at least one gluon as an external particle, we have used the Ward identity test for every phase space point. Higher precision has been used to recompute events which fail the gauge-invariance check. As a second test we have verified cancelation of infrared poles. We have also cross-checked our results with the publicly available MADGRAPH5_AMC@NLO code [59]. More specifically we have compared 


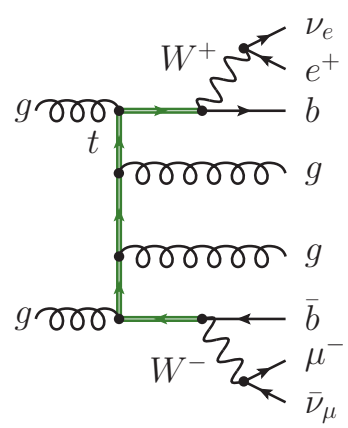

(a)

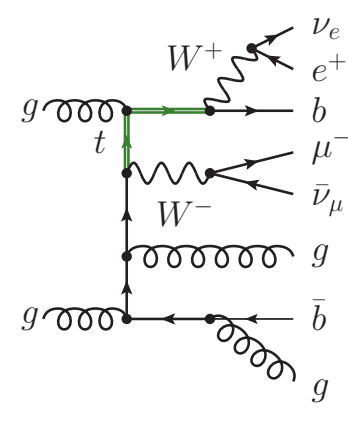

(b)

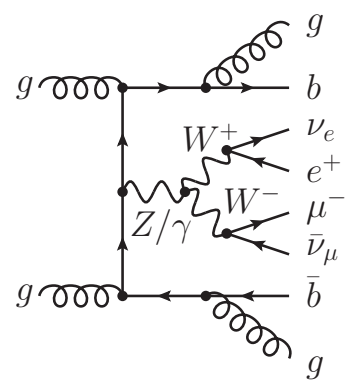

(c)

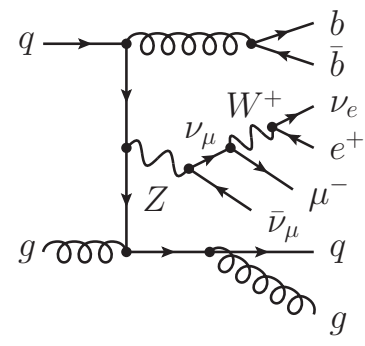

$(d)$

Figure 3. A representative set of Feynman diagrams involving two (a), one (b) and no top-quark resonances (c and d) contributing to the real emission corrections to the $p p \rightarrow e^{+} \nu_{e} \mu^{-} \bar{\nu}_{\mu} b \bar{b} j+X$ process at $\mathcal{O}\left(\alpha_{s}^{4} \alpha^{4}\right)$.

results for the virtual NLO contribution to the squared amplitude, $2 \Re\left(\mathcal{M}_{\text {tree }}^{*} \mathcal{M}_{\text {one-loop }}\right)$, for a few phase-space points. The calculation of the virtual corrections has been achieved with the help of the package Helac-1Loop [60] which incorporates CuTTools [61] and OneLOop [62] as its cornerstones. The first code contains an implementation of the OPP method for the reduction of one-loop amplitudes at the integrand level [63-66], while the second one is dedicated to the evaluation of the one-loop scalar functions. Renormalisation is done, as usual, by evaluating tree-level diagrams with counterterms. For our process, we chose to renormalise the coupling in the $\overline{\mathrm{MS}}$ scheme with five active flavours and the top quark decoupled. The mass renormalisation is performed in the on-shell scheme.

The real emission corrections to the LO process arise from tree-level amplitudes with one additional parton, i.e. an additional gluon, or a quark anti-quark pair replacing a gluon. For the calculation of the real emission contributions, the package HeLAC-DiPOLEs has been employed. It implements the massless dipole formalism of Catani and Seymour [67], as well as its massive version as developed by Catani, Dittmaier, Seymour and Trocsanyi [68], for arbitrary helicity eigenstates and colour configurations of the external partons [52]. Moreover, a new subtraction formalism, first introduced by Nagy and Soper in the formulation of an improved parton shower [69, 70], is also included in the framework. The Nagy-Soper subtraction scheme [71] makes use of random polarisation and colour sampling of the external partons. A phase space restriction on the contribution of the subtraction terms is included for both subtraction cases. Also for the real corrections, we adopt the KALEU phase-space generator that is equipped with additional, special channels that proved to be important for phase-space optimisation. All possible subprocesses contributing to the real emission part can be classified into various categories presented in table 2, together with the number of Feynman diagrams, the Catani-Seymour dipoles and the Nagy-Soper subtraction terms corresponding to each subprocess. Typical examples of the real emission graphs are displayed in figure 3. Having two independent subtraction schemes available, we were able to cross check the correctness of the real corrections by comparison between the two results. 


\begin{tabular}{|c|c|c|c|}
\hline $\begin{array}{l}\text { PARTONIC } \\
\text { SubProcess }\end{array}$ & $\begin{array}{c}\text { Number Of } \\
\text { FEYNMAN Diagrams }\end{array}$ & $\begin{array}{l}\text { Number Of } \\
\text { CS Dipoles }\end{array}$ & $\begin{array}{c}\text { Number Of } \\
\text { NS Subtractions }\end{array}$ \\
\hline$g g \rightarrow e^{+} \nu_{e} \mu^{-} \bar{\nu}_{\mu} b \bar{b} g g$ & 4447 & 56 & 14 \\
\hline$g g \rightarrow e^{+} \nu_{e} \mu^{-} \bar{\nu}_{\mu} b \bar{b} q \bar{q}$ & 1952 & 40 & 10 \\
\hline$g q \rightarrow e^{+} \nu_{e} \mu^{-} \bar{\nu}_{\mu} b \bar{b} g q$ & 1952 & 40 & 10 \\
\hline$g \bar{q} \rightarrow e^{+} \nu_{e} \mu^{-} \bar{\nu}_{\mu} b \bar{b} g \bar{q}$ & 1952 & 40 & 10 \\
\hline$q \bar{q} \rightarrow e^{+} \nu_{e} \mu^{-} \bar{\nu}_{\mu} b \bar{b} g g$ & 1952 & 40 & 10 \\
\hline$q q \rightarrow e^{+} \nu_{e} \mu^{-} \bar{\nu}_{\mu} b \bar{b} q q$ & 930 & 20 & 5 \\
\hline$q \bar{q} \rightarrow e^{+} \nu_{e} \mu^{-} \bar{\nu}_{\mu} b \bar{b} q \bar{q}$ & 930 & 16 & 4 \\
\hline $\bar{q} \bar{q} \rightarrow e^{+} \nu_{e} \mu^{-} \bar{\nu}_{\mu} b \bar{b} \bar{q} \bar{q}$ & 930 & 20 & 5 \\
\hline$q q^{\prime} \rightarrow e^{+} \nu_{e} \mu^{-} \bar{\nu}_{\mu} b \bar{b} q q^{\prime}$ & 501 & 12 & 3 \\
\hline$q \bar{q} \rightarrow e^{+} \nu_{e} \mu^{-} \bar{\nu}_{\mu} b \bar{b} q^{\prime} \bar{q}^{\prime}$ & 501 & 8 & 2 \\
\hline$q \bar{q}^{\prime} \rightarrow e^{+} \nu_{e} \mu^{-} \bar{\nu}_{\mu} b \bar{b} q \bar{q}^{\prime}$ & 501 & 12 & 3 \\
\hline $\bar{q} \bar{q}^{\prime} \rightarrow e^{+} \nu_{e} \mu^{-} \bar{\nu}_{\mu} b \bar{b} \bar{q} \bar{q}^{\prime}$ & 501 & 12 & 3 \\
\hline$q Q \rightarrow e^{+} \nu_{e} \mu^{-} \bar{\nu}_{\mu} b \bar{b} q Q$ & 465 & 12 & 3 \\
\hline$q \bar{q} \rightarrow e^{+} \nu_{e} \mu^{-} \bar{\nu}_{\mu} b \bar{b} Q \bar{Q}$ & 465 & 8 & 2 \\
\hline$q \bar{Q} \rightarrow e^{+} \nu_{e} \mu^{-} \bar{\nu}_{\mu} b \bar{b} q \bar{Q}$ & 465 & 12 & 3 \\
\hline $\bar{q} \bar{Q} \rightarrow e^{+} \nu_{e} \mu^{-} \bar{\nu}_{\mu} b \bar{b} \bar{q} \bar{Q}$ & 465 & 12 & 3 \\
\hline$q Q \rightarrow e^{+} \nu_{e} \mu^{-} \bar{\nu}_{\mu} b \bar{b} q^{\prime} Q^{\prime}$ & 36 & 4 & 1 \\
\hline$q \bar{Q} \rightarrow e^{+} \nu_{e} \mu^{-} \bar{\nu}_{\mu} b \bar{b} q^{\prime} \bar{Q}^{\prime}$ & 36 & 4 & 1 \\
\hline$q \bar{q}^{\prime} \rightarrow e^{+} \nu_{e} \mu^{-} \bar{\nu}_{\mu} b \bar{b} Q \bar{Q}^{\prime}$ & 36 & 4 & 1 \\
\hline $\bar{q} \bar{Q} \rightarrow e^{+} \nu_{e} \mu^{-} \bar{\nu}_{\mu} b \bar{b} \bar{q}^{\prime} \bar{Q}^{\prime}$ & 36 & 4 & 1 \\
\hline$g g \rightarrow e^{+} \nu_{e} \mu^{-} \bar{\nu}_{\mu} b \bar{b} b \bar{b}$ & 3904 & 48 & 12 \\
\hline$q \bar{q} \rightarrow e^{+} \nu_{e} \mu^{-} \bar{\nu}_{\mu} b \bar{b} b \bar{b}$ & 930 & 16 & 4 \\
\hline
\end{tabular}

Table 2. The list of partonic subprocesses contributing to the subtracted real emissions for the $p p \rightarrow e^{+} \nu_{e} \mu^{-} \bar{\nu}_{\mu} b \bar{b} j+X$ process. Also shown are the number of Feynman diagrams, as well as the number of Catani-Seymour and Nagy-Soper subtraction terms. Notation: $q$ and $\bar{q}$ stands for $u p-$ or down-type quark, $Q$ and $\bar{Q}$ denotes charm or strange quark.

Finally, since the produced top quarks are unstable particles, the inclusion of the decays is performed in the complex mass scheme $[43,72,73]$, which respects gauge invariance. At the amplitude level (at LO and NLO) we simply incorporate $\Gamma_{t}$ into the definition of the squared top-quark mass as follows

$$
\mu_{t}^{2}=m_{t}^{2}-i m_{t} \Gamma_{t},
$$

where $\mu_{t}^{2}$ is identified with the position of the pole of the top-quark propagator. All matrix elements are, thus, evaluated using complex masses and the top-quark mass counter-term 
$\delta \mu_{t}$ is related to the top-quark self-energy at $p_{t}^{2}=\mu_{t}^{2}$. Another non trivial aspect of this substitution consists of the evaluation of one-loop scalar integrals in the presence of complex masses. In our case this part is done by ONELOOP, which supports complex masses.

To summarise, our computational system is based on Helac-1Loop and HelaCDipoles, which are both parts of the HELAC-NLO MC program [74]. The framework relies upon a number of optimizations designed to speed-up the evaluation of the virtual and real corrections while keeping an acceptable numerical precision. Prominent examples are the use of Monte Carlo sampling over colour configurations and polarisations/helicities and reweighting techniques for the calculation of the virtual part, or the adoption of a phase space restriction for the calculation of the real-emission part. All these techniques have been extensively used and proved their efficiency in our previous calculations [42, 75-80]. At the same time, given the complexity of the current project, it has been necessary to extend our computational framework with new functionalities and improvements which proved essential for the feasibility of the calculation. Without putting too much weight on technical details, one relevant improvement concerns the optimisation of the algorithms for the generation of the skeleton files, which store all the necessary information for the calculation of the amplitudes in the HeLAC-NLO system. To be more precise, skeletons contain the full set of instructions for the recursive evaluation of amplitudes according to the Dyson-Schwinger algorithm, together with relevant accessory information such as the number of external particles, flavour assignments and colour-connection configurations. This information is evaluated in the form of integers and stored once for all in skeleton files during the so-called initialisation phase. In the subsequent phase, skeletons are read to provide the instructions to return the actual value of the amplitude. For more details we refer the interested reader to our previous publications [54, 74]. It should be clear that achieving an efficient generation of skeletons is the fundamental prerequisite for the whole calculation. Typically, the combinatorics of diagram topologies become quickly very complex when the number of external particles increases. In the HELAC-NLO software, a top-down approach is used to obtain all currents needed in the Dyson-Schwinger recursive representation of the amplitude. All possible vertices are first scanned in order to select all non-zero sub-amplitudes. Afterwards the program checks whether the selected subamplitudes are indeed contributing to the final amplitude under consideration. The number of loop topologies rapidly increases with the number of external particles and puts serious challenges starting from $2 \rightarrow 5$ processes, where the efficient selection and bookkeeping of topologies becomes a critical issue for the feasibility of the calculation. To this end a few optimizations have been introduced for the generation of the skeleton files in the HeLAC1LOOP program. The most relevant comprises an introduction of the additional filter which performs a pre-selection of topologies based on the information of particles that are attached to the loop. In this way, assuming a specific model, which is the Standard Model in our case, a large fraction of configurations allowed by combinatorics can be just discarded a priori without need to go through subsequent and more time-consuming steps that scan individual vertices. A similar approach has also been applied to the treatment of tree-level processes by looking at the external particle content in each Dyson-Schwinger current. This increases dramatically the efficiency of the generation of skeleton files also in HeLAC- 


\begin{tabular}{|lcccc|}
\hline Contribution & Nr. of Events & NR. OF FILES & (AVG) EVEnts/File & SizE \\
\hline Born & $21 \times 10^{6}$ & 60 & $350 \times 10^{3}$ & $38 \mathrm{~GB}$ \\
Born + Virtual & $33 \times 10^{6}$ & 380 & $87 \times 10^{3}$ & $72 \mathrm{~GB}$ \\
Integrated dipoles & $80 \times 10^{6}$ & 450 & $178 \times 10^{3}$ & $160 \mathrm{~GB}$ \\
Real + Sub. Real & $626 \times 10^{6}$ & 18000 & $35 \times 10^{3}$ & $1250 \mathrm{~GB}$ \\
\hline Total: & $760 \times 10^{6}$ & 18890 & $40 \times 10^{3}$ & $1520 \mathrm{~GB}$ \\
\hline
\end{tabular}

Table 3. Number of events, number of files and the averaged number of events per file as well as the total size per contribution for the different Ntuple samples for the $p p \rightarrow e^{+} \nu_{e} \mu^{-} \bar{\nu}_{\mu} b \bar{b} j+X$ process.

Dipoles. Finally, we have exploited the fact that the procedure of computing skeleton files consists of several independent modules for each loop topology (i.e. heptagons, hexagons etc.) and colour-connection configuration. Thus, it is possible to perform parallel runs that are dedicated to the individual pieces of the skeleton file. All parts are put together in the end, which reduces considerably the computing time. Using these optimisations we have achieved a reduction of one order of magnitude in the generation of skeleton files for the process under consideration. Another improvement in HELAC-NLO is the implementation of a new option for selecting automatically the desired perturbative order in $\alpha$ and $\alpha_{s}$, preserving at the same time the structure and the advantages of the DysonSchwinger recursion algorithm for the construction of the amplitudes. This modification is particularly useful for our project given that we are interested in mixed contributions, i.e. $\mathcal{O}\left(\alpha_{s}^{3} \alpha^{4}\right)$ at LO and $\mathcal{O}\left(\alpha_{s}^{4} \alpha^{4}\right)$ at NLO. The modifications summarised above make the calculation feasible. Due to high demands in terms of CPU time it is, however, very expensive to repeat the calculation for different choices of scales and PDFs, as is required for a proper assessment of the theoretical uncertainties. To be able to study scale and PDF uncertainties in a timely manner, we have made use of unweighting techniques to produce event samples for the central scale and PDF set, which are then reweighted to get results for different sets of scales and PDFs. To be more precise, building on [81] we have implemented in Helac-NLO the apparatus for the generation of Ntuples of events. The Ntuple format shows a clear advantage for changing kinematical cuts or observables, which can be obtained without need of any additional rerunning of the code. Furthermore, any change in scales or PDFs can be accommodated by simple reweighting, provided that the necessary matrixelement information is stored in the Ntuples. Table 3 summarises the total number of Ntuple files and their sizes, which have been generated for the present analysis. Except for the virtual part, which is obtained by reweighting of the (unweighted) Born events, the Ntuples contain unweighted events. In this way we have minimised the number of events stored in these files. With the goal of optimising the performance of the unweighting, we have implemented the so-called partial unweighting in HELAC-NLO. Instead of looking for the maximal weight to perform the unweighting according to its value we have decided to choose some approximate $w_{\max }$. All events with a weight $w$ lower than a given threshold $w_{\max }$ have been unweighted up to this threshold, while for events with $w>w_{\max }$ the event 
weights, i.e. $w / w_{\max }$, have been kept. In the end both types of events, i.e. events with $w=1$ and $w \neq 1$, have to be evaluated together to give a final cross section and its error. This procedure has proved particularly helpful for the process at hand, where when using the standard unweighting procedure some shortcomings are encountered, as elaborated in the following. Before the unweighting procedure is performed, a preunweighting phase is done to find the maximum weight. In order to find the correct maximal weight a huge number of events need to be evaluated, which for such complicated final state is time consuming. If the correct maximum weight is found, which typically is a very large number comparing to the average weight, the unweighting procedure becomes extremely inefficient. Moreover, if during the preunweighting phase the maximum weight found is not the correct one, the unweighting procedure is spoilt. As a consequence various differential distributions close to their peaks are not properly described. The partial unweighting helps to bypass these problems while giving the exact answer at the same time. In practice, to find the approximate maximal weight we use 200000 accepted events in the preunweighting phase. This typically results in about $1 \%-10 \%$ of the total number of events carrying a non-unit weight.

\section{Results for the LHC Run II energy of $13 \mathrm{TeV}$}

\subsection{Numerical setup}

We consider the process $p p \rightarrow e^{+} \nu_{e} \mu^{-} \bar{\nu}_{\mu} b \bar{b} j+X$ for the LHC Run II energy of $\sqrt{s}=13 \mathrm{TeV}$. We only simulate decays of the weak bosons to different lepton generations to avoid virtual photon singularities stemming from quasi-collinear $\gamma^{*} \rightarrow \ell^{ \pm} \ell^{\mp}$ decays. These interference effects are at the per-mille level for inclusive cuts, as checked by an explicit leading order calculation. The complete cross section with $\ell=e, \mu$ can be obtained by multiplying the result with a lepton-flavor factor of 4 . The Cabibbo-Kobayashi-Maskawa (CKM) mixing of the quark generations is neglected, i.e. the CKM matrix has a diagonal form. The SM parameters are given in the $G_{\mu}$ scheme

$$
\begin{aligned}
G_{\mu} & =1.16637 \cdot 10^{-5} \mathrm{GeV}^{-2}, & m_{t} & =173.2 \mathrm{GeV}[82], \\
m_{W} & =80.399 \mathrm{GeV}, & \Gamma_{W} & =2.09875 \mathrm{GeV}, \\
m_{Z} & =91.1876 \mathrm{GeV}, & \Gamma_{Z} & =2.50848 \mathrm{GeV} .
\end{aligned}
$$

The electromagnetic coupling is derived from the Fermi constant $G_{\mu}$ according to

$$
\alpha=\frac{\sqrt{2}}{\pi} G_{\mu} m_{W}^{2} \sin ^{2} \theta_{W},
$$

where $\sin ^{2} \theta_{W}$ is the weak mixing angle defined as

$$
\sin ^{2} \theta_{W}=1-\frac{m_{W}^{2}}{m_{Z}^{2}} .
$$

Since we are interested in NLO QCD corrections, electroweak gauge bosons are treated within the fixed width scheme, thus, we use the real $W$ and $Z$ boson masses and also 
$\sin ^{2} \theta_{W}$ is kept real. Masses of all other particles (leptons and quarks), including the bottom quark, are set to zero. We have checked using the integrated cross section at LO that finite bottom-quark mass effects lead to a reduction of the cross section by less than $1 \%$. The width of the top quark for an unstable $W$ boson and the massless bottom quark according to [83] is given by

$$
\Gamma_{t}^{\mathrm{LO}}=1.47834 \mathrm{GeV}, \quad \Gamma_{t}^{\mathrm{NLO}}=1.35146 \mathrm{GeV} .
$$

Since we treat bottom quarks as massless partons there are no diagrams with Higgs boson exchange at tree level. We also neglect closed fermion loops involving top quarks coupled to the Higgs boson. Following recommendations of PDF4LHC for the usage of parton distribution functions (PDFs) suitable for applications at the LHC Run II [84] we employ CT14 [85], MMHT14 [86] and NNPDF3.0 [87] sets. In particular, we take CT14nlo, NNPDF3.0-nlo-as-0118 and MMHT14nlo68clas118 at NLO as well as CT14llo, NNPDF3.0lo-as-0130 and MMHT14lo68cl at LO. The running of the strong coupling constant $\alpha_{s}$ with two-loop (one-loop) accuracy at NLO (LO) is provided by the LHAPDF interface [88]. The number of active flavours is $N_{F}=5$. Contributions induced by the bottom-quark parton density are neglected. We have determined that for the integrated LO cross section neglecting the bottom-quark contribution to PDFs amounts to less than $0.1 \%$. We use the corresponding prescription from each group to provide the $68 \%$ confidence level (C.L.) PDF uncertainties. Both CT14 PDFs and MMHT14 PDFs include a central set and error sets in the Hessian representation. In that case we use the asymmetric expression for PDF uncertainties [88]. For an observable $\mathcal{O}$, given a central PDF member $S_{0}$ and $2 N$ eigenvector PDF members $S_{i}^{ \pm}(i=1, \ldots, N)$, uncertainties are given by

$$
\begin{aligned}
\delta \mathcal{O}_{\mathrm{PDF}+} & =\sqrt{\sum_{i=1}^{N}\left[\max \left(\mathcal{O}\left(S_{i}^{+}\right)-\mathcal{O}\left(S_{0}\right), \mathcal{O}\left(S_{i}^{-}\right)-\mathcal{O}\left(S_{0}\right), 0\right)\right]^{2}}, \\
\delta \mathcal{O}_{\mathrm{PDF}-} & =\sqrt{\sum_{i=1}^{N}\left[\max \left(\mathcal{O}\left(S_{0}\right)-\mathcal{O}\left(S_{i}^{+}\right), \mathcal{O}\left(S_{0}\right)-\mathcal{O}\left(S_{i}^{-}\right), 0\right)\right]^{2}} .
\end{aligned}
$$

Let us note that for CT14 and MMHT14 we have $2 N=56$ and $2 N=50$ respectively. Additionally, the CT14 errors are rescaled by a factor $1 / 1.645$ since they are provided at 90\% C.L. On the other hand NNPDF3.0 PDFs uses the Monte Carlo sampling method in conjunction with neural networks. In that case PDF uncertainties are obtained using the replicas method defined by

$$
\delta \mathcal{O}_{\mathrm{PDF}+}=\delta \mathcal{O}_{\mathrm{PDF}-}=\delta \mathcal{O}_{\mathrm{PDF}}=\sqrt{\frac{1}{N-1} \sum_{i=1}^{N}\left[\mathcal{O}\left(S_{i}\right)-\mathcal{O}\left(S_{0}\right)\right]^{2}},
$$

where a set of $N=100$ Monte Carlo PDF members $S_{i}(i=1, \ldots, N)$ has been used. We also have

$$
\mathcal{O}\left(S_{0}\right)=\langle\mathcal{O}\rangle=\frac{1}{N} \sum_{i=1}^{N} \mathcal{O}\left(S_{i}\right)
$$


such that $\delta \mathcal{O}_{\mathrm{PDF}}$ can be rewritten as

$$
\delta \mathcal{O}_{\mathrm{PDF}}=\sqrt{\frac{N}{N-1}\left[\left\langle\mathcal{O}^{2}\right\rangle-\langle\mathcal{O}\rangle^{2}\right]} .
$$

Our calculation, like any fixed-order one, contains a residual dependence on the renormalisation scale, $\mu_{R}$, and the factorisation scale, $\mu_{F}$, arising from the truncation of the perturbative expansion. As a consequence, observables depend on the values of $\mu_{R}$ and $\mu_{F}$ that are provided as input parameters. We assume that the default scale $\mu_{R}=\mu_{F}=\mu_{0}$ is the same for both the renormalisation and factorisation scales. The scale systematics, however, is evaluated by varying $\mu_{R}$ and $\mu_{F}$ independently in the range

$$
\frac{1}{2} \mu_{0} \leq \mu_{R}, \mu_{F} \leq 2 \mu_{0}, \quad \frac{1}{2} \leq \frac{\mu_{R}}{\mu_{F}} \leq 2,
$$

which in practise amounts to consider the following pairs

$$
\left(\frac{\mu_{R}}{\mu_{0}}, \frac{\mu_{F}}{\mu_{0}}\right)=\{(2,1),(0.5,1),(1,2),(1,1),(1,0.5),(2,2),(0.5,0.5)\} .
$$

We search for the minimum and maximum of the resulting cross section. Let us mention here that while calculating the scale dependence for the NLO cross section we keep $\Gamma_{t}^{\mathrm{NLO}}$ fixed independently of the scale choice. The error introduced by this treatment is however of higher orders. We have checked that for the $p p \rightarrow e^{+} \nu_{e} \mu^{-} \bar{\nu}_{\mu} b \bar{b}+X$ production process, which is a simpler case, and for two scales $\mu=0.5 \mu_{0}$ and $\mu=2 \mu_{0}$ with $\mu_{0}=m_{t}$ it amounts to $\pm 1.5 \%$ deviation, respectively [42]. As a natural scale for the process we choose the mass of the heaviest particle appearing in the process, that is the top-quark mass and set $\mu_{0}=m_{t}$. Total cross sections are mostly influenced by final-state production relatively close to the threshold as defined by particle masses, which justifies our choice. However, differential cross sections extend up to energy scales that are much larger than the threshold, and show larger shape distortions in such high-energy regions [40]. Therefore, we examine two additional choices, namely $\mu_{0}=E_{T} / 2$ and $\mu_{0}=H_{T} / 2$, where $E_{T}$ and $H_{T}$ are defined as

$$
\begin{aligned}
& E_{T}=m_{T, t}+m_{T, \bar{t}}=\sqrt{m_{t}^{2}+p_{T, t}^{2}}+\sqrt{m_{t}^{2}+p_{T, \bar{t}}^{2}} \\
& H_{T}=p_{T, e^{+}}+p_{T, \mu^{-}}+p_{T, j_{b_{1}}}+p_{T, j_{b_{2}}}+\not p_{T}+p_{T, j_{1}} .
\end{aligned}
$$

Here $t$ and $\bar{t}$ are reconstructed from their decay products, albeit we use bottom-jets denoted as $j_{b_{1}}$ and $j_{b_{2}}$ not bottom quarks in the reconstruction. Additionally, $j_{1}$ is the first hardest light-jet (jets are ordered in $p_{T}$ ) and $\not p_{T}=\left|\mathbf{p}_{T, \nu_{e}}+\mathbf{p}_{T, \bar{\nu}_{\mu}}\right|$ is the total missing transverse momentum from escaping neutrinos. Let us note here, that for small values of $p_{T, t}$ and $p_{T, \bar{t}}$, i.e. close to the $t \bar{t}$ threshold, $E_{T} / 2 \approx m_{t}$. All final state partons with pseudorapidity $|\eta|<5$, where $\eta=-\ln (\tan \theta / 2)$, are recombined into jets via the IR-safe anti- $k_{T}$ jet algorithm [89] with the separation parameter in the rapidity-azimuthal-angle plane set to $R=0.5$. We require exactly two bottom-jets, at least one light-jet, two charged leptons and non-zero missing transverse momentum $\not p_{T}$. These final states have to fullfil the following 
criteria, which we consider to be very inclusive selection cuts

$$
\begin{aligned}
p_{T, \ell} & >30 \mathrm{GeV}, & p_{T, j} & >40 \mathrm{GeV}, \\
p_{T} & >40 \mathrm{GeV}, & \Delta R_{j j} & >0.5, \\
\Delta R_{\ell \ell} & >0.4, & \Delta R_{\ell j} & >0.4, \\
\left|y_{\ell}\right| & <2.5, & \left|y_{j}\right| & <2.5,
\end{aligned}
$$

where $\ell$ stands for $\mu^{-}, e^{+}$and $j$ corresponds to light- and bottom-jets. Additionally, the transverse momentum, $p_{T, i}$, rapidity, $y_{i}$, as well as the separation in the rapidity-azimuthalangle-plane, $\Delta R_{i k}$, where $i, k=\ell, j$ are defined as

$$
\begin{aligned}
p_{T, i} & =\sqrt{p_{x, i}^{2}+p_{y, i}^{2}}, \\
y_{i} & =\frac{1}{2} \ln \left(\frac{E_{i}+p_{z, i}}{E_{i}-p_{z, i}}\right), \\
\Delta R_{i k} & =\sqrt{\Delta \phi_{i k}^{2}+\Delta y_{i k}^{2}} .
\end{aligned}
$$

\subsection{Integrated cross sections with theoretical uncertainties}

We begin the presentation of our results with a discussion of the integrated cross section using the scale choice $\mu_{F}=\mu_{R}=\mu_{0}=m_{t}$. We define the upper and the lower limit of the scale variation according to eq. (3.10) and the PDF uncertainties are considered to be at the $\pm 1 \sigma$ level ( $68 \%$ C.L.). Our results for the integrated cross section with the CT14 PDF sets and $\mu_{0}=m_{t}$ are as follows

$$
\begin{aligned}
& \sigma_{e^{+} \nu_{e} \mu^{-} \bar{\nu}_{\mu} b \bar{b} j}^{\mathrm{LO}}\left(\mathrm{CT} 14, \mu_{0}=m_{t}\right)=608.09_{-188.85}^{+303.52(-31 \%)}(+50 \%)[\text { scales }] \mathrm{fb}, \\
& \sigma_{e^{+} \nu_{e} \mu^{-} \bar{\nu}_{\mu} b \bar{b} j}^{\mathrm{NLO}}\left(\mathrm{CT} 14, \mu_{0}=m_{t}\right)=537.24_{-190.35(-35 \%)}^{+10.12(+2 \%)}[\mathrm{scales}]_{-18.34(-3 \%)}^{+17.32(+3 \%)}[\mathrm{PDF}] \mathrm{fb} \text {. }
\end{aligned}
$$

For the MMHT14 PDF sets we have obtained instead

$$
\begin{aligned}
& \sigma_{e^{+} \nu_{e} \mu^{-} \bar{\nu}_{\mu} b \bar{b} j}^{\mathrm{LO}}\left(\mathrm{MMHT} 14, \mu_{0}=m_{t}\right)=665.58_{-216.08(-32 \%)}^{+357.64(+54 \%)}[\text { scales }] \mathrm{fb} \\
& \sigma_{e^{+} \nu_{e} \mu^{-} \bar{\nu}_{\mu} b \bar{b} j}^{\mathrm{NLM}}\left(\mathrm{MMT} 14, \mu_{0}=m_{t}\right)=542.56_{-106.46(-20 \%)}^{+10.02(+2 \%)}[\text { scales }]_{-11.33(-2 \%)}^{+12.31(+2 \%)}[\mathrm{PDF}] \mathrm{fb} .
\end{aligned}
$$

And finally, with the NNPDF3.0 PDF sets our results read

$$
\begin{aligned}
& \sigma_{e^{+} \nu_{e} \mu^{-} \bar{\nu}_{\mu} b \bar{b} j}^{\mathrm{LO}}\left(\mathrm{NNPDF} 3.0, \mu_{0}=m_{t}\right)=582.29_{-184.75(-32 \%)}^{+302.06(+52 \%)}[\mathrm{scales}] \mathrm{fb} \\
& \sigma_{e^{+} \nu_{e} \mu^{-} \bar{\nu}_{\mu} b \bar{b} j}^{\mathrm{NLN}}\left(\mathrm{NNDF} 3.0, \mu_{0}=m_{t}\right)=559.66_{-111.05(-20 \%)}^{+10.64(+2 \%)}[\mathrm{scales}]_{-8.42(-2 \%)}^{+8.42(+2 \%)}[\mathrm{PDF}] \mathrm{fb} .
\end{aligned}
$$

A few comments are in order. To start, at the central value of the fixed scale, i.e. for $\mu_{0}=m_{t}$, we obtain negative and moderate NLO corrections, which are of the order of $12 \%$ for the CT14 PDF set, $18 \%$ for MMHT14 and 4\% for NNPDF3.0. Defining scale uncertainties in a very conservative way, using the lower and upper bounds of our results, gives us an estimate of $50 \%$ for the LO prediction, independent of the PDF set. After inclusion 
of the NLO QCD corrections, they are reduced down to about $20 \%$ for MMHT14 PDF and NNPDF3.0. In case of CT14 PDF the reduction is smaller and the final theoretical uncertainties are at the $35 \%$ level. However in the case of truly asymmetric uncertainties it is always more appropriate to symmetrise the errors. After symmetrisation the scale uncertainty at LO is assessed to be instead of the order of $40 \%$. After inclusion of the NLO QCD corrections, the scale uncertainty is reduced down to $11 \%$ for NNPDF3.0 and MMHT14 and $18 \%$ for CT14. Another source of uncertainties comes from the PDF parametrisation. We calculate these uncertainties as explained in the previous section according to eq. (3.5) and eq. (3.6). They amount to $\pm 3 \%$ for CT14 and $\pm 2 \%$ for MMHT14 and NNPDF3.0. These numbers refer to the uncertainties at the $68 \%$ C.L. for the individual PDF sets, but do not take into account additional systematics coming from the underlying assumptions that enter the parametrisation of different PDF sets, which cannot be quantified within a given scheme. We see that CT14, MMHT14 and NNPDF3.0 NLO results differ by $1 \%-4 \%$, which is comparable to the individual estimates of PDF systematics. Overall, the PDF uncertainties for the process under scrutiny are well below the theoretical uncertainties due to the scale dependence, which remain the dominant source of the theoretical systematics. In table 4 we report the total cross section at LO and NLO for different cuts on the transverse momentum of the hardest light-jet, $p_{T, j_{1}}$. Theoretical uncertainties coming from scale variation, denoted as $\delta_{\text {scale }}$, and from PDFs, denoted as $\delta_{\mathrm{PDF}}$ together with a $\mathcal{K}$-factor defined as $\sigma^{\mathrm{NLO}} / \sigma^{\mathrm{LO}}$ are additionally presented in the table 4 . Within each PDF set we observe a very stable behaviour of systematics when varying the $p_{T, j_{1}}$ cut within the $40-120 \mathrm{GeV}$ range. NLO corrections are also quite stable, changing the $\mathcal{K}$-factor by less than 7\%,5\% and 4\% for CT14, MMHT14 and NNPDF3.0 respectively.

In the following we examine two choices for a dynamical factorisation and renormalisation scale. As a first scale we adopt $\mu_{R}=\mu_{F}=\mu_{0}=E_{T} / 2$, where $E_{T}$ is defined in eq. (3.11). Our second choice is $\mu_{R}=\mu_{F}=\mu_{0}=H_{T} / 2$, where $H_{T}$ is the sum of the transverse momenta of all final state objects (bottom and light jets, missing transverse momentum and charged leptons) according to eq. (3.11). We repeat the same analysis performed in the previous case, where we considered the fixed scale $\mu_{0}=m_{t}$. We start with results for $\mu_{R}=\mu_{F}=\mu_{0}=E_{T} / 2$ and the CT14 PDF set, which are as follows

$$
\begin{aligned}
& \sigma_{e^{+} \nu_{e} \mu^{-} \bar{\nu}_{\mu} b \bar{b} j}^{\mathrm{LO}}\left(\mathrm{CT} 14, \mu_{0}=E_{T} / 2\right)=493.54_{-147.02(+30 \%)}^{+230.40(+47 \%)}[\text { scales }] \mathrm{fb} \\
& \sigma_{e^{+} \nu_{e} \mu^{-} \bar{\nu}_{\mu} b \bar{b} j}^{\mathrm{NLO}}\left(\mathrm{CT} 14, \mu_{0}=E_{T} / 2\right)=544.64_{-117.47(+22 \%)}^{+2.95}\left({ }_{(-2 \%)}^{+1 \%}\right)[\mathrm{scales}]_{-18.92(+3 \%)}^{+18.10(+3 \%)}[\mathrm{PDF}] \mathrm{fb} .
\end{aligned}
$$

For the MMHT14 PDF set we have the following integrated cross sections

$$
\begin{aligned}
& \sigma_{e^{+} \nu_{e} \mu^{-} \bar{\nu}_{\mu} b \bar{b} j}^{\mathrm{LO}}\left(\mathrm{MMHT} 14, \mu_{0}=E_{T} / 2\right)=536.43_{-166.94(-31 \%)}^{+268.93(+50 \%)}[\text { scales }] \mathrm{fb} \\
& \sigma_{e^{+} \nu_{e} \mu^{-} \bar{\nu}_{\mu} b \bar{b} j}^{\mathrm{NL} j}\left(\mathrm{MMHT} 14, \mu_{0}=E_{T} / 2\right)=549.58_{-49.90}^{+3.11(+1 \%)}[\text { scales }]_{-11.61(-2 \%)}^{+12.74(+2 \%)}[\mathrm{PDF}] \mathrm{fb},
\end{aligned}
$$




\begin{tabular}{|c|c|c|c|c|c|c|c|}
\hline PDF & $p_{T, j_{1}}$ & $\sigma^{\mathrm{LO}}[\mathrm{fb}]$ & $\delta_{\text {scale }}$ & $\sigma^{\mathrm{NLO}}[\mathrm{fb}]$ & $\delta_{\text {scale }}$ & $\delta_{\mathrm{PDF}}$ & $\mathcal{K}$ \\
\hline \multirow[t]{6}{*}{$\mathrm{CT}$} & 40 & 608.09 & \multirow{6}{*}{$\begin{array}{l}+303.52(+50 \%) \\
-188.85(-31 \%) \\
+220.20(+51 \%) \\
-136.12(-31 \%) \\
+170.40(+52 \%) \\
-104.76(-33 \%) \\
+136.64(+52 \%) \\
-83.60(-32 \%) \\
+112.14(+53 \%) \\
-68.31(-32 \%)\end{array}$} & 537.24 & \multirow{6}{*}{$\begin{array}{l}+10.12(+2 \%) \\
-190.35(-35 \%) \\
+6.35(+2 \%) \\
-127.14(-33 \%) \\
+4.78(+2 \%) \\
-93.77(-32 \%) \\
+4.01(+2 \%) \\
-73.36(-33 \%) \\
+3.57(+2 \%) \\
-59.58(-34 \%)\end{array}$} & \multirow{6}{*}{$\begin{array}{l}+17.32(+3 \%) \\
-18.34(-3 \%) \\
+13.20(+3 \%) \\
-13.54(-4 \%) \\
+10.44(+4 \%) \\
-10.41(-4 \%) \\
+8.41(+4 \%) \\
-8.18(-4 \%) \\
+6.88(+4 \%) \\
-6.53(-4 \%)\end{array}$} & 0.88 \\
\hline & 60 & 433.47 & & 384.35 & & & 0.89 \\
\hline & & & & 等 & & & \\
\hline & 80 & 330.55 & & 289.15 & & & 0.87 \\
\hline & 100 & 261.65 & & 223.70 & & & 0.85 \\
\hline & 120 & 212.23 & & 176.05 & & & 0.83 \\
\hline \multirow[t]{6}{*}{ MMHT } & 40 & 665.58 & \multirow{6}{*}{$\begin{array}{l}+357.64(+54 \%) \\
-216.08(-32 \%) \\
+257.33(+55 \%) \\
-154.52(-33 \%) \\
+197.80(+55 \%) \\
-118.17(-33 \%) \\
+157.69(+56 \%) \\
-93.78(-33 \%) \\
+128.76(+57 \%) \\
-76.26(-34 \%)\end{array}$} & 542.56 & \multirow{6}{*}{$\begin{array}{l}+10.02(+2 \%) \\
-106.46(-20 \%) \\
+6.25(+2 \%) \\
-73.95(-19 \%) \\
+4.71(+2 \%) \\
-58.23(-20 \%) \\
+3.95(+2 \%) \\
-49.17(-22 \%) \\
+3.54(+2 \%) \\
-43.14(-24 \%)\end{array}$} & \multirow{6}{*}{$\begin{array}{l}+12.31(+2 \%) \\
-11.33(-2 \%) \\
+8.97(+2 \%) \\
-8.15(-2 \%) \\
+6.83(+2 \%) \\
-6.18(-2 \%) \\
+5.34(+2 \%) \\
-4.82(-2 \%) \\
+4.25(+2 \%) \\
-3.84(-2 \%)\end{array}$} & 0.82 \\
\hline & 60 & 471.36 & & 387.34 & & & 082 \\
\hline & & & & & & & \\
\hline & 80 & 357.55 & & 290.91 & & & 0.81 \\
\hline & 100 & 281.75 & & 224.75 & & & 0.80 \\
\hline & 120 & 227.63 & & 176.59 & & & 0.78 \\
\hline \multirow[t]{6}{*}{ NNPDF } & 40 & 582.29 & \multirow{6}{*}{$\begin{array}{l}+302.06(+52 \%) \\
-184.75(-32 \%) \\
+216.23(+53 \%) \\
-131.50(-32 \%) \\
+165.46(+53 \%) \\
-100.15(-32 \%) \\
+131.35(+54 \%) \\
-79.19(-32 \%) \\
+106.82(+54 \%) \\
-64.16(-33 \%)\end{array}$} & 559.66 & \multirow{6}{*}{$\begin{array}{l}+10.64(+2 \%) \\
-111.05(-20 \%) \\
+6.64(+2 \%) \\
-77.17(-19 \%) \\
+4.99(+2 \%) \\
-60.79(-20 \%) \\
+4.19(+2 \%) \\
-51.35(-22 \%) \\
+3.74(+2 \%) \\
-45.06(-25 \%)\end{array}$} & \multirow{6}{*}{$\begin{array}{l}+8.42(+2 \%) \\
-8.42(-2 \%) \\
+6.06(+2 \%) \\
-6.06(-2 \%) \\
+4.64(+2 \%) \\
-4.64(-2 \%) \\
+3.67(+2 \%) \\
-3.67(-2 \%) \\
+2.97(+2 \%) \\
-2.97(-2 \%)\end{array}$} & 0.96 \\
\hline & 60 & 410.73 & & 399.81 & & & 0.97 \\
\hline & 80 & 310.50 & & 300.39 & & & 0.97 \\
\hline & & & & & & & \\
\hline & & & & & & & \\
\hline & 120 & 196.46 & & 182.46 & & & 0.93 \\
\hline
\end{tabular}

Table 4. Integrated cross section for the $p p \rightarrow e^{+} \nu_{e} \mu^{-} \bar{\nu}_{\mu} b \bar{b} j+X$ production process at the LHC with $\sqrt{s}=13 \mathrm{TeV}$. Results are evaluated using $\mu_{R}=\mu_{F}=\mu_{0}=m_{t}$ for three different PDF sets and five different $p_{T, j_{1}}$ cuts for the hardest light-jet. Also given are theoretical uncertainties coming from scale variation, $\delta_{\text {scale }}$, and from PDFs, $\delta_{\mathrm{PDF}}$. In the last column a $\mathcal{K}$-factor is shown.

while for the NNPDF3.0 set we have

$$
\begin{aligned}
& \sigma_{e^{+} \nu_{e} \mu^{-} \bar{\nu}_{\mu} b \bar{b} j}^{\mathrm{LO}}\left(\mathrm{NNPDF} 3.0, \mu_{0}=E_{T} / 2\right)=473.88_{-144.34(-30 \%)}^{+223.00(+47 \%)}[\text { scales] fb } \\
& \sigma_{e^{+} \nu_{e} \mu^{-} \bar{\nu}_{\mu} b \bar{b} j}^{\mathrm{NLN}}\left(\mathrm{NNDF} 3.0, \mu_{0}=E_{T} / 2\right)=567.13_{-51.53(+9 \%)}^{+3.15(+1 \%)}\left[\text { scales] }{ }_{-8.63(-2 \%)}^{+8.63(+2 \%)}[\mathrm{PDF}] \mathrm{fb} .\right.
\end{aligned}
$$

The use of the dynamical instead of the fixed scale hardly affects the NLO integrated cross section. For each PDF set, a difference of only $1.5 \%$ is observed. On the other hand the LO cross sections are lowered by more than $20 \%$, which results in positive NLO corrections. The size of the latter, however, remains the same, i.e. it varies between $2 \%-20 \%$ depending on the PDF set. Additionally, PDF uncertainties are of the same size. The integrated NLO cross sections are shifted by maximally $4 \%$ when different PDF sets are used, which again remains within the uncertainties of the individual set. Theoretical uncertainties at LO taken conservatively (after symmetrisation) have been estimated to be around 50\% (40\%) and at NLO they are reduced down to $22 \%$ (11\%) for CT14 and to 10\% (5\%) for MMHT14 and NNPDF3.0 sets. These conclusions are not affected by the variation of the $p_{T, j_{1}}$ cut, that we move within the $40-120 \mathrm{GeV}$ range as can been seen from table 5 . Lastly, for our 


\begin{tabular}{|c|c|c|c|c|c|c|c|}
\hline PDF & $p_{T, j_{1}}$ & $\sigma^{\mathrm{LO}}[\mathrm{fb}]$ & $\delta_{\text {scale }}$ & $\sigma^{\mathrm{NLO}}[\mathrm{fb}]$ & $\delta_{\text {scale }}$ & $\delta_{\mathrm{PDF}}$ & $\mathcal{K}$ \\
\hline \multirow[t]{6}{*}{$\mathrm{CT}$} & 40 & 493.54 & \multirow{6}{*}{$\begin{array}{l}+230.40(+47 \%) \\
-147.02(-30 \%) \\
+164.28(+47 \%) \\
-104.31(-30 \%) \\
+125.07(+48 \%) \\
-79.10(-30 \%) \\
+98.69(+48 \%) \\
-62.20(-30 \%) \\
+79.69(+49 \%) \\
-50.08(-31 \%)\end{array}$} & 544.64 & \multirow{6}{*}{$\begin{array}{l}+2.95(+1 \%) \\
-117.47(-22 \%) \\
+3.23(+1 \%) \\
-75.76(-20 \%) \\
+2.80(+1 \%) \\
-54.31(-19 \%) \\
+2.27(+1 \%) \\
-41.32(-18 \%) \\
+1.76(+1 \%) \\
-32.72(-18 \%)\end{array}$} & \multirow{6}{*}{$\begin{array}{l}+18.10(+3 \%) \\
-18.92(-3 \%) \\
+13.67(+4 \%) \\
-13.87(-4 \%) \\
+10.79(+4 \%) \\
-10.63(-4 \%) \\
+8.73(+4 \%) \\
-8.39(-4 \%) \\
+7.17(+4 \%) \\
-6.73(-4 \%)\end{array}$} & 1.10 \\
\hline & 60 & 347.04 & & 387.25 & & & 1.12 \\
\hline & 80 & 26126 & & 20083 & & & 111 \\
\hline & 80 & 261.26 & & 290.83 & & & 1.11 \\
\hline & 100 & 204.16 & & 225.43 & & & 1.10 \\
\hline & 120 & 163.48 & & 178.04 & & & 1.09 \\
\hline \multirow{7}{*}{ MMHT } & & & \multirow{7}{*}{$\begin{array}{l}+268.93(+50 \%) \\
-166.94(-31 \%) \\
+190.06(+51 \%) \\
-117.46(-31 \%) \\
+143.64(+51 \%) \\
-88.46(-32 \%) \\
+112.61(+52 \%) \\
-69.13(-32 \%) \\
+90.41(+52 \%) \\
-55.36(-32 \%)\end{array}$} & & \multirow{7}{*}{$\begin{array}{l}+3.11(+1 \%) \\
-49.90(-9 \%) \\
+5.04(+1 \%) \\
-37.67(-10 \%) \\
+4.13(+1 \%) \\
-28.79(-10 \%) \\
+2.74(+1 \%) \\
-22.26(-10 \%) \\
+1.79(+1 \%) \\
-17.26(-10 \%)\end{array}$} & \multirow{7}{*}{$\begin{array}{l}+12.74(+2 \%) \\
-11.61(-2 \%) \\
+9.20(+2 \%) \\
-8.33(-2 \%) \\
+7.01(+2 \%) \\
-6.32(-2 \%) \\
+5.51(+2 \%) \\
-4.95(-2 \%) \\
+4.41(+2 \%) \\
-3.97(-2 \%)\end{array}$} & \\
\hline & 40 & 536.43 & & 549.58 & & & 1.02 \\
\hline & 60 & 374.58 & & 389.97 & & & 1.04 \\
\hline & 80 & 280.38 & & 292.39 & & & 1.04 \\
\hline & 100 & 21801 & & 2063 & & & 104 \\
\hline & 100 & 218.01 & & 220.33 & & & 1.04 \\
\hline & 120 & 173.79 & & 178.48 & & & 1.03 \\
\hline \multirow[t]{6}{*}{ NNPDF } & 40 & 473.88 & \multirow{6}{*}{$\begin{array}{l}+223.00(+47 \%) \\
-144.34(-30 \%) \\
+161.85(+49 \%) \\
-101.15(-31 \%) \\
+121.86(+50 \%) \\
-75.91(-31 \%) \\
+95.18(+50 \%) \\
-59.14(-31 \%) \\
+76.15(+50 \%) \\
-47.21(-31 \%)\end{array}$} & 567.13 & \multirow{6}{*}{$\begin{array}{l}+3.15(+1 \%) \\
-51.53(-9 \%) \\
+5.20(+1 \%) \\
-38.96(-10 \%) \\
+4.26(+1 \%) \\
-29.80(-10 \%) \\
+2.82(+1 \%) \\
-23.05(-10 \%) \\
+1.83(+1 \%) \\
-17.88(-10 \%)\end{array}$} & \multirow{6}{*}{$\begin{array}{l}+8.63(+2 \%) \\
-8.63(-2 \%) \\
+6.21(+2 \%) \\
-6.21(-2 \%) \\
+4.76(+2 \%) \\
-4.76(-2 \%) \\
+3.79(+2 \%) \\
-3.79(-2 \%) \\
+3.09(+2 \%) \\
-3.09(-2 \%)\end{array}$} & 1.20 \\
\hline & & & & & & & \\
\hline & 60 & & & & & & 1.22 \\
\hline & 80 & 246.17 & & 302.03 & & & 1.23 \\
\hline & 100 & 190.91 & & 233.86 & & & 1.22 \\
\hline & 120 & 151.82 & & 184.48 & & & 1.22 \\
\hline
\end{tabular}

Table 5. Integrated cross section for the $p p \rightarrow e^{+} \nu_{e} \mu^{-} \bar{\nu}_{\mu} b \bar{b} j+X$ production process at the LHC with $\sqrt{s}=13 \mathrm{TeV}$. Results are evaluated using $\mu_{R}=\mu_{F}=\mu_{0}=E_{T} / 2$ for three different PDF sets and five different $p_{T, j_{1}}$ cuts for the hardest light-jet. Also given are theoretical uncertainties coming from scale variation, $\delta_{\text {scale }}$, and from PDFs, $\delta_{\mathrm{PDF}}$. In the last column a $\mathcal{K}$-factor is shown.

third choice of scale, $\mu_{R}=\mu_{F}=\mu_{0}=H_{T} / 2$ and for the CT14 PDF set we can write

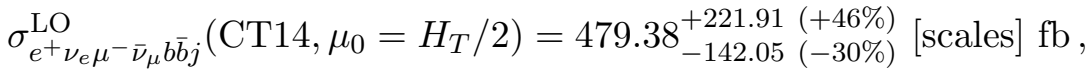

$$
\begin{aligned}
& \sigma_{e^{+} \nu_{e} \mu^{-} \bar{\nu}_{\mu} b \bar{b} j}^{\mathrm{NLO}}\left(\mathrm{CT} 14, \mu_{0}=H_{T} / 2\right)=549.65_{-53.42(-10 \%)}^{+10.25(+2 \%)}\left[\text { scales }{ }_{-19.15(-3 \%)}^{+18.00(+3 \%)}[\mathrm{PDF}] \mathrm{fb}\right. \text {. }
\end{aligned}
$$

For the MMHT14 PDF set we obtain

$$
\begin{aligned}
& \sigma_{e^{+} \nu_{e} \mu^{-} \bar{\nu}_{\mu} b \bar{b} j}^{\mathrm{LO}}\left(\mathrm{MMHT} 14, \mu_{0}=H_{T} / 2\right)=521.08_{-161.36(-31 \%)}^{+259.12(+50 \%)}[\text { scales }] \mathrm{fb}, \\
& \sigma_{e^{+} \nu_{e} \mu^{-} \bar{\nu}_{\mu} b \bar{b} j}^{\mathrm{NLO}}\left(\mathrm{MMHT} 14, \mu_{0}=H_{T} / 2\right)=554.61_{-54.51(-10 \%)}^{+10.85\left({ }_{(-2 \%)}\right.}[\mathrm{scales}]_{-12.22(+2 \%)}^{+12.06(+2 \%)}[\mathrm{PDF}] \mathrm{fb} \text {, }
\end{aligned}
$$

and for the NNPDF3.0 PDF set our integrated cross section are as follows

$$
\begin{aligned}
& \sigma_{e^{+} \nu_{e} \mu^{-} \bar{\nu}_{\mu} b \bar{b} j}^{\mathrm{LO}}\left(\mathrm{NNPDF} 3.0, \mu_{0}=H_{T} / 2\right)=460.80_{-139.68(-30 \%)}^{+221.93(+48 \%)}[\text { scales }] \mathrm{fb} \\
& \sigma_{e^{+} \nu_{e} \mu^{-} \bar{\nu}_{\mu} b \bar{b} j}^{\mathrm{NLO}}\left(\mathrm{NNPDF} 3.0, \mu_{0}=H_{T} / 2\right)=572.18_{-56.23(-10 \%)}^{+11.14\left({ }_{(-2 \%)}\right.}[\text { scales }]_{-11.31(-2 \%)}^{+11.31(+2 \%)}[\mathrm{PDF}] \mathrm{fb} .
\end{aligned}
$$




\begin{tabular}{|c|c|c|c|c|c|c|c|}
\hline PDF & $p_{T, j_{1}}$ & $\sigma^{\mathrm{LO}}[\mathrm{fb}]$ & $\delta_{\text {scale }}$ & $\sigma^{\mathrm{NLO}}[\mathrm{fb}]$ & $\delta_{\text {scale }}$ & $\delta_{\mathrm{PDF}}$ & $\mathcal{K}$ \\
\hline \multirow[t]{5}{*}{$\mathrm{CT}$} & 40 & 479.38 & \multirow{5}{*}{$\begin{array}{l}+221.91(+46 \%) \\
-142.05(-30 \%) \\
+153.04(+47 \%) \\
-97.75(-30 \%) \\
+113.00(+47 \%) \\
-72.05(-30 \%) \\
+86.79(+47 \%) \\
-55.26(-30 \%) \\
+68.43(+47 \%) \\
-43.52(-30 \%)\end{array}$} & 549.65 & \multirow{5}{*}{$\begin{array}{l}+10.25(+2 \%) \\
-53.42(-10 \%) \\
+11.93(+3 \%) \\
-40.33(-10 \%) \\
+11.23(+4 \%) \\
-31.57(-11 \%) \\
+9.61(+4 \%) \\
-24.96(-11 \%) \\
+7.90(+5 \%) \\
-19.90(-11 \%)\end{array}$} & \multirow{5}{*}{$\begin{array}{l}+18.00(+3 \%) \\
-19.15(-3 \%) \\
+13.43(+3 \%) \\
-13.91(-4 \%) \\
+10.50(+4 \%) \\
-10.66(-4 \%) \\
+8.43(+4 \%) \\
-8.37(-4 \%) \\
+6.88(+4 \%) \\
-6.71(-4 \%)\end{array}$} & 1.15 \\
\hline & 60 & 328.60 & & 384.37 & & & 1.17 \\
\hline & 80 & 241.43 & & 286.68 & & & 1.19 \\
\hline & 100 & 184.69 & & 221.01 & & & 1.20 \\
\hline & 120 & 145.11 & & 173.90 & & & 1.20 \\
\hline \multirow[t]{6}{*}{ MMHT } & 40 & 521.08 & \multirow{6}{*}{$\begin{array}{l}+259.12(+50 \%) \\
-161.36(-31 \%) \\
+176.68(+50 \%) \\
-109.89(-31 \%) \\
+129.23(+50 \%) \\
-80.30(-31 \%) \\
+98.44(+50 \%) \\
-61.13(-31 \%) \\
+77.05(+50 \%) \\
-47.83(-31 \%)\end{array}$} & 554.61 & \multirow{6}{*}{$\begin{array}{l}+10.85(+2 \%) \\
-54.51(-10 \%) \\
+12.30(+3 \%) \\
-40.98(-11 \%) \\
+11.50(+4 \%) \\
-31.99(-11 \%) \\
+9.77(+4 \%) \\
-25.24(-11 \%) \\
+8.01(+5 \%) \\
-20.08(-12 \%)\end{array}$} & \multirow{6}{*}{$\begin{array}{l}+12.06(+2 \%) \\
-12.22(-2 \%) \\
+8.58(+2 \%) \\
-8.84(-2 \%) \\
+6.43(+2 \%) \\
-6.81(-2 \%) \\
+4.98(+2 \%) \\
-5.42(-2 \%) \\
+3.94(+2 \%) \\
-4.40(-2 \%)\end{array}$} & 1.06 \\
\hline & 60 & 354.08 & & 386.98 & & & 1.09 \\
\hline & 80 & 258.31 & & 288.13 & & & 1.12 \\
\hline & & & & 22180 & & & 1.13 \\
\hline & 100 & 196.39 & & & & & 1.15 \\
\hline & 120 & 153.47 & & 174.28 & & & 1.14 \\
\hline \multirow[t]{6}{*}{ NNPDF } & 40 & 460.80 & \multirow{6}{*}{$\begin{array}{l}+221.93(+48 \%) \\
-139.68(-30 \%) \\
+150.81(+48 \%) \\
-94.83(-30 \%) \\
+109.97(+48 \%) \\
-69.10(-30 \%) \\
+83.53(+48 \%) \\
-52.47(-30 \%) \\
+65.20(+48 \%) \\
-40.95(-30 \%)\end{array}$} & 572.18 & \multirow{6}{*}{$\begin{array}{l}+11.14(+2 \%) \\
-56.23(-10 \%) \\
+12.74(+3 \%) \\
-42.42(-11 \%) \\
+11.92(+4 \%) \\
-33.13(-11 \%) \\
+10.13(+4 \%) \\
-26.15(-11 \%) \\
+8.31(+5 \%) \\
-20.82(-12 \%)\end{array}$} & \multirow{6}{*}{$\begin{array}{l}+11.31(+2 \%) \\
-11.31(-2 \%) \\
+9.15(+2 \%) \\
-9.15(-2 \%) \\
+7.40(+2 \%) \\
-7.40(-2 \%) \\
+6.01(+3 \%) \\
-6.01(-3 \%) \\
+5.06(+3 \%) \\
-5.06(-3 \%)\end{array}$} & 1.24 \\
\hline & 60 & 312.34 & & 399.61 & & & 1.28 \\
\hline & & & & & & & \\
\hline & 80 & 227.37 & & 297.04 & & & 1.31 \\
\hline & 100 & 172.51 & & 229.19 & & & 1.33 \\
\hline & 120 & 134.57 & & 180.15 & & & 1.34 \\
\hline
\end{tabular}

Table 6. Integrated cross section for the $p p \rightarrow e^{+} \nu_{e} \mu^{-} \bar{\nu}_{\mu} b \bar{b} j+X$ production process at the LHC with $\sqrt{s}=13 \mathrm{TeV}$. Results are evaluated using $\mu_{R}=\mu_{F}=\mu_{0}=H_{T} / 2$ for three different PDF sets and five different $p_{T, j_{1}}$ cuts for the hardest light-jet. Also given are theoretical uncertainties coming from scale variation, $\delta_{\text {scale }}$, and from PDFs, $\delta_{\mathrm{PDF}}$. In the last column a $\mathcal{K}$-factor is shown.

The behaviour of the integrated cross section with the $\mu_{0}=H_{T} / 2$ scale choice is similar to $\mu_{0}=E_{T} / 2$. At the central value of the scale positive and moderate NLO QCD corrections have been obtained. Specifically, we observe 15\% corrections for the CT14 PDF set, $6 \%$ for MMHT and $24 \%$ for the NNPDF set. Moreover PDF uncertainties are of the same size, $2 \%-3 \%$ only. The only visible difference is the magnitude of theoretical uncertainties due to the scale variation. For the last choice, i.e. $H_{T} / 2$, we have not only obtained the smallest theoretical error, but this error remains the same independently of the PDF set used. Namely, LO uncertainties that are of the order of $50 \%$ (40\%) are cut down to $10 \%(6 \%)$ at NLO, independently of the PDF set, where for values in the brackets the symmetrisation of errors is performed. Results are also quite stable when shifting the $p_{T, j_{1}}$ cut from $40 \mathrm{GeV}$ up to $120 \mathrm{GeV}$ as presented in table 6 . For $120 \mathrm{GeV} p_{T, j_{1}}$-cut, the NLO scale dependence increases by $2 \%$ only up to $12 \%$ (8\%) respectively. Even if the scale choices $E_{T} / 2$ and $H_{T} / 2$ have similar features, the latter leads to the smallest theoretical errors and is therefore best suited for the calculation of cross sections within the scope of our analysis.

To illustrate why the two dynamical scale choices give similar results we plot in figure 4 differential cross section distributions as a function of $E_{T}$ and $H_{T}$. The left panel displays 

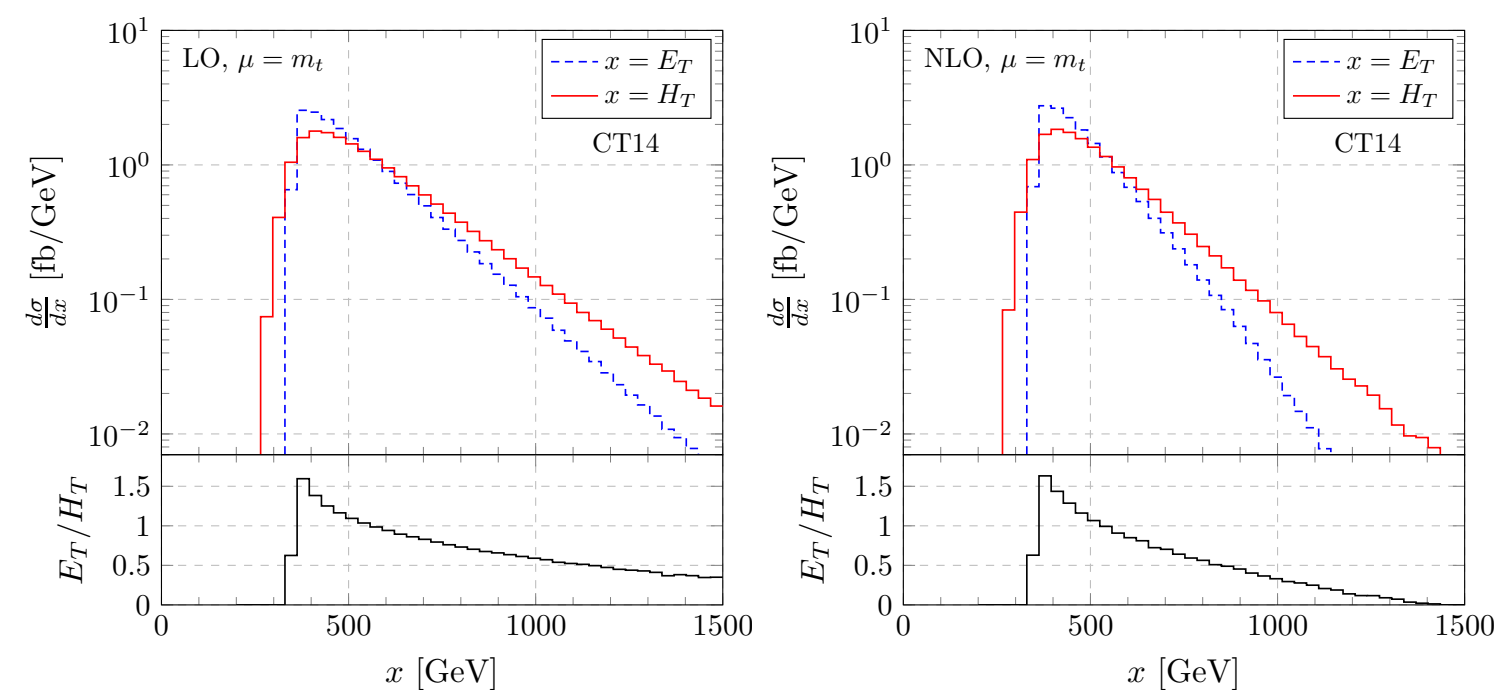

Figure 4. Differential cross section distribution as a function of $E_{T}$ and $H_{T}$ at LO (left panel) and at NLO (right panel) for the $p p \rightarrow e^{+} \nu_{e} \mu^{-} \bar{\nu}_{\mu} b \bar{b} j+X$ process at the LHC run II with $\sqrt{s}=13 \mathrm{TeV}$. Renormalisation and factorisation scales are set to the common value $\mu_{R}=\mu_{F}=\mu_{0}=m_{t}$. The LO and the NLO CT14 PDF sets are employed. Also shown is the $E_{T} / H_{T}$ ratio.

LO results whereas the right panel NLO ones. Renormalisation and factorisation scales are set to the common (fixed) value $\mu_{R}=\mu_{F}=\mu_{0}=m_{t}$ and the CT14 LO and NLO PDF sets have been employed. The upper panels present observables while the lower panels display the $E_{T} / H_{T}$ ratio. With our selection cuts the $H_{T}$ distribution has its maximum around $2 m_{t}$. Moreover, both observables are quite similar in the region close to the $t \bar{t}$ threshold and up to about $750 \mathrm{GeV}$, which influences the total integrated cross section. Above $750 \mathrm{GeV}$ the $H_{T}$ spectrum is much harder than the corresponding $E_{T}$ spectrum, which should be reflected in the high $p_{T}$ tails of various differential cross sections that we are going to examine in the next section.

It is also instructive to present the scale dependence of our results in a more graphical fashion. To this end, we show in figure 5 the total cross sections at LO and NLO, based on the CT14 PDF set. The scales $\mu_{R}$ and $\mu_{F}$ are varied simultaneously according to the prescription $\mu_{R}=\mu_{F}=\mu_{0}=\xi m_{t}$ with $\xi \in(0.125, \ldots, 8)$. The dependence is large, illustrating the well known fact that the LO prediction can only provide a rough estimate. At the LHC with $\sqrt{s}=13 \mathrm{TeV}$ for $\xi=1$ and for our selection cut the $g g$ channel (blue dashed curve) dominates the total $p p$ cross section by about $72 \%$, followed by the $g q$ channel (black dashed curve) with about 18\%. The remaining 10\% comes from two additional channels, $g \bar{q}$ (green dashed curve) and $q \bar{q}$ (pink dashed curve) that contributes at the $6 \%$ and $4 \%$ level respectively. In the right panel of figure 5 the scale dependence of the NLO cross section (red curves) is shown together with the LO one (blue curves). This time for three different scale choices, namely $\mu_{0}=m_{t}, \mu_{0}=E_{T} / 2$ and $\mu_{0}=H_{T} / 2$. As already discussed, we observe a reduction of the scale uncertainty while going from LO to NLO. Additionally, we confirm that both $\mu_{0}=E_{T} / 2$ and $\mu_{0}=H_{T} / 2$ give similar results within the whole plotted range. In figure 6 we display again the dependence of the integrated LO 

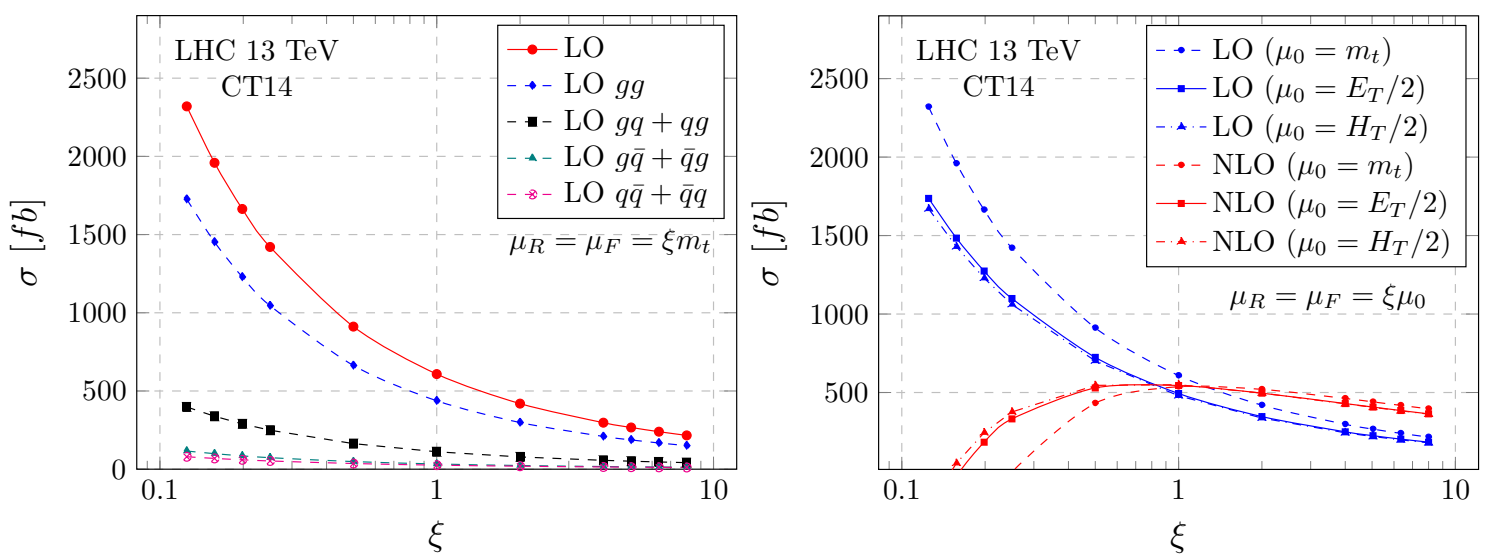

Figure 5. Scale dependence of the LO cross section with the individual contributions of the partonic channels (left panel) and scale dependence of the LO and NLO cross sections (right panel) for the $p p \rightarrow e^{+} \nu_{e} \mu^{-} \bar{\nu}_{\mu} b \bar{b} j+X$ process at the LHC run II with $\sqrt{s}=13 \mathrm{TeV}$. Renormalisation and factorisation scales are set to the common value $\mu_{R}=\mu_{F}=\mu_{0}$ with $\mu_{0}=m_{t}, \mu_{0}=H_{T} / 2$ and $\mu_{0}=E_{T} / 2$. The LO and the NLO CT14 PDF sets are employed.

(black dashed curve) and NLO (red solid curve) cross sections on the variation of the fixed and dynamical scales for the CT14 PDF set. Here, however, we show additionally results with individual variation of $\mu_{R}$ and $\mu_{F}$. Each time we plot two additional curves, the first one (green solid curve) corresponds to the case where $\mu_{R}$ is kept fix at the central value, while $\mu_{F}$ is varied and the second one (blue dashed curve) describes the opposite situation. We can observe that, independently of the scale choice, either it is $\mu_{0}=m_{t}, \mu_{0}=E_{T} / 2$ or $\mu_{0}=H_{T} / 2$, the scale variation is driven by the changes in $\mu_{R}$.

To summarise this part, for the total cross section, where effects of the phase space regions close to the threshold for the $t \bar{t}$ production dominate, all three scales, $\mu_{0}=m_{t}$, $\mu_{0}=E_{T} / 2$ and $\mu_{0}=H_{T} / 2$ describe the process under scrutiny very well. They all agree within their respective theoretical errors, as it should be, however, $\mu_{0}=H_{T} / 2$ provides the smallest theoretical error, that is independent of the PDF set and the $p_{T, j_{1}}$ cut applied. For this reason, it can be recommended as the best one for the computation of total cross sections for inclusive analyses at $\sqrt{s}=13 \mathrm{TeV}$. On the other hand, differential cross sections extend themselves up to energy scales that are much larger than the $t \bar{t}$ threshold. Thus, in the next section we shall examine which scales are also suitable for the description of differential cross sections.

\subsection{Differential distributions}

In addition to the normalization of the integrated cross section, QCD corrections can affect the shape of various kinematic distributions. To quantify the size of these distortions we shall examine differential distributions for various observables of interest for the LHC. These distributions are obtained with the CT14 PDF sets by applying the cuts and parameters specified in the previous section. Also here we examine three different scale choices, the fixed scale $\mu_{0}=m_{t}$ and two dynamical scales $\mu_{0}=E_{T} / 2$ and $\mu_{0}=H_{T} / 2$. For each of the observables we present three plots that correspond to the three scale choices. The 


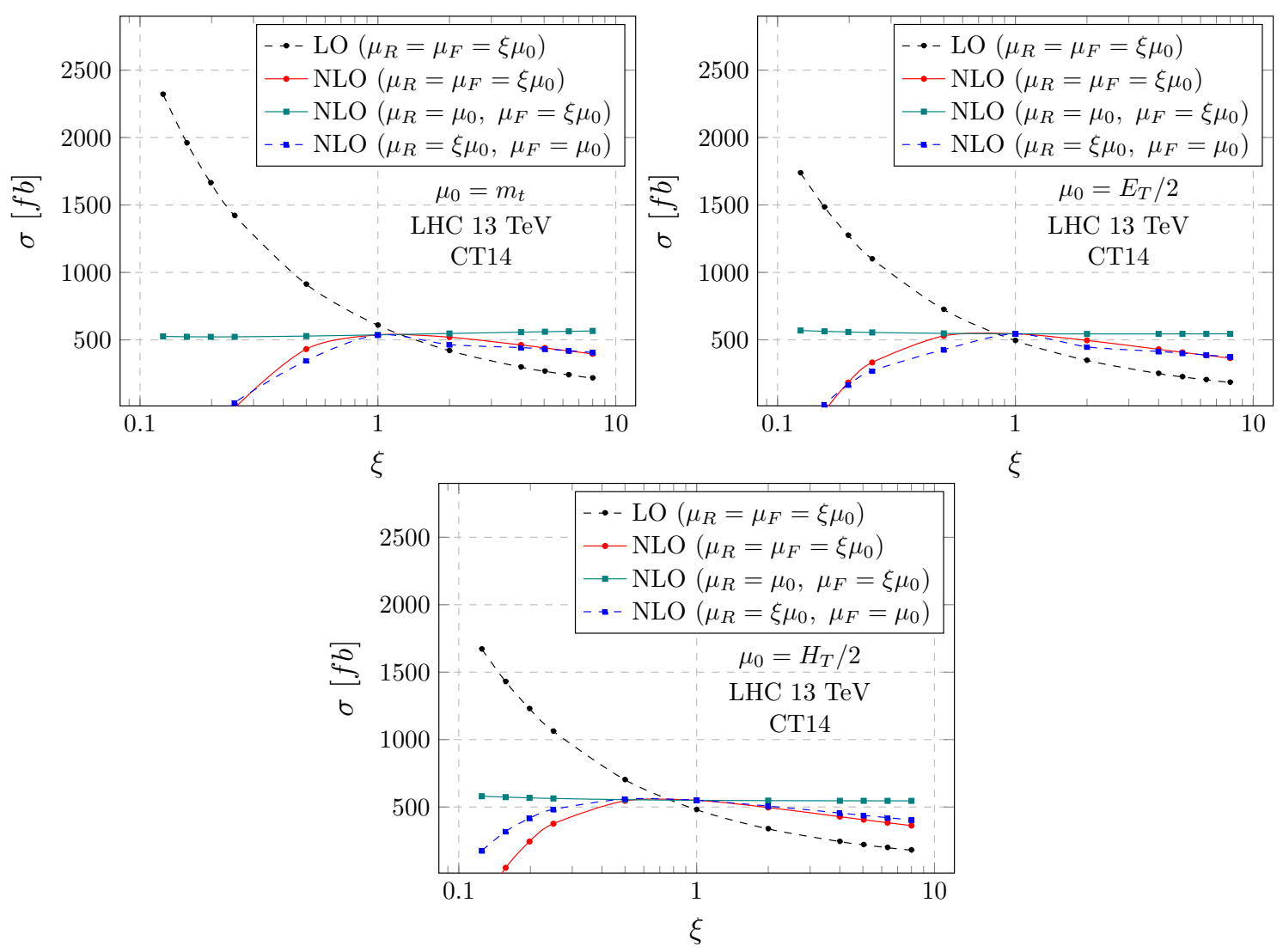

Figure 6. Scale dependence of the LO and NLO integrated cross section for the $p p \rightarrow$ $e^{+} \nu_{e} \mu^{-} \bar{\nu}_{\mu} b \bar{b} j+X$ process at the LHC run II with $\sqrt{s}=13 \mathrm{TeV}$. Renormalisation and factorisation scales are set to the common value $\mu_{R}=\mu_{F}=\mu_{0}$ with $\mu_{0}=m_{t}, \mu_{0}=E_{T} / 2$ and $\mu_{0}=H_{T} / 2$. The LO and the NLO CT14 PDF sets are employed. For each case of $\mu_{0}$ also shown is the variation of $\mu_{R}$ with fixed $\mu_{F}$ and the variation of $\mu_{F}$ with fixed $\mu_{R}$.

upper panel of each plot shows the absolute prediction at LO and NLO together with their scale dependence bands obtained from the envelope of results calculated according to eq. (3.10). The lower panels display the same LO and NLO predictions normalised to the LO result at the central scale. Thus, the blue band provides the relative scale uncertainty of the LO cross section, whereas the red band gives the differential $\mathcal{K}$-factor together with the uncertainty band.

We start with the top-quark kinematics. In figures 7 and 9 we present the averaged differential cross section as a function of the transverse momentum and rapidity of the top quark. In figure 11 the invariant mass of the $t \bar{t}$ system, $M_{t \bar{t}}=\sqrt{\left(p_{t}+p_{\bar{t}}\right)^{2}}$, is plotted. The kinematics of the top quark and top antiquark are determined from the four-momenta of final state objects, i.e. leptons and bottom-jets. The $p_{T, t}$ and $y_{t}$ distributions are especially useful to validate and tune a given parton shower model as well as to check specific higher order QCD calculations. They can also be exploited in searches for physics beyond the SM. On the other hand, the invariant mass of the $t \bar{t}$ pair is the observable to look for new $s$-channel resonances that may arise in the $t \bar{t}$ system. It can be used to test new 


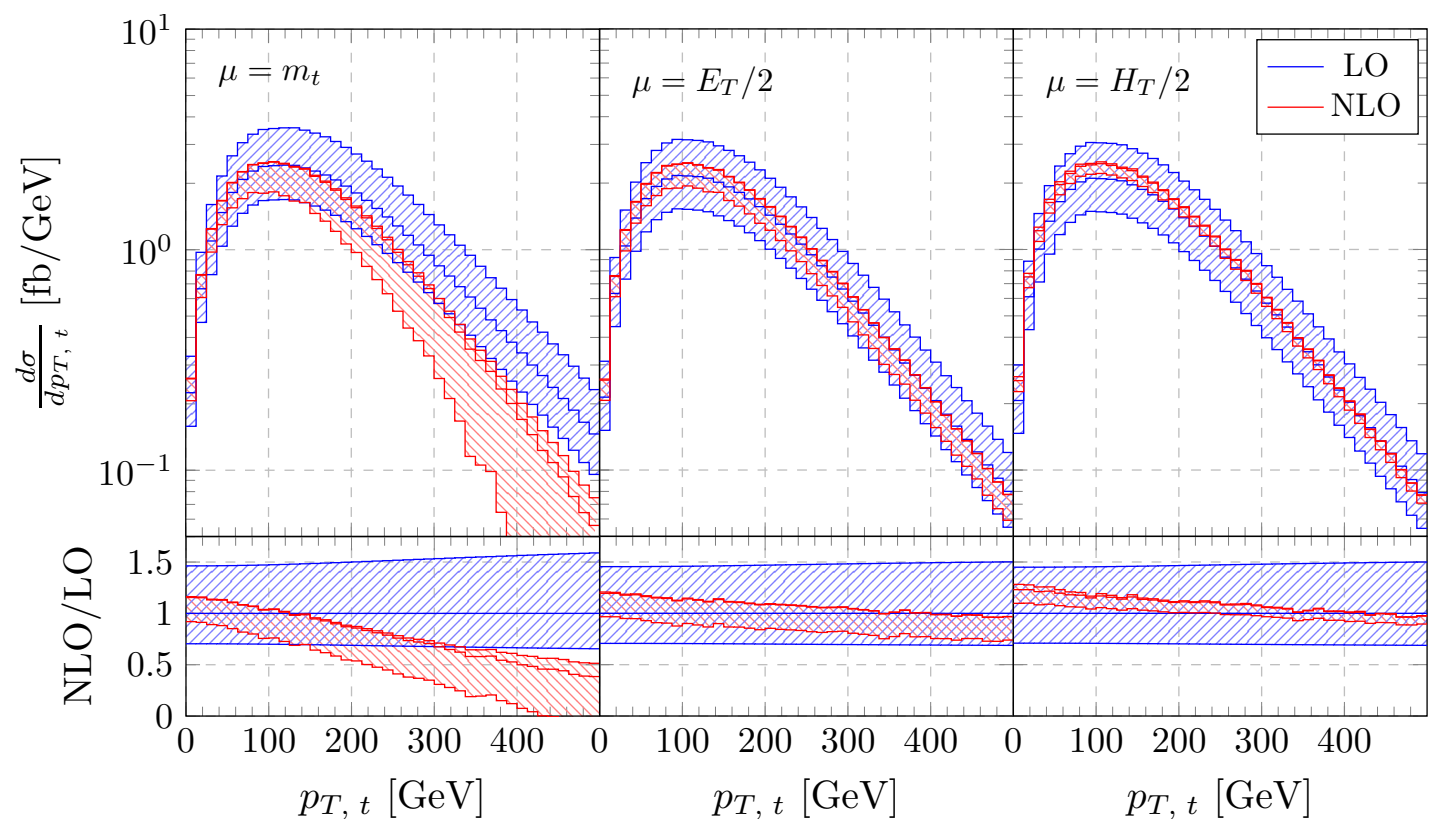

Figure 7. Averaged differential cross section distributions as a function of the transverse momentum of the top quark for the $p p \rightarrow e^{+} \nu_{e} \mu^{-} \bar{\nu}_{\mu} b \bar{b} j+X$ process at the LHC run II with $\sqrt{s}=13 \mathrm{TeV}$. The upper plot shows absolute LO and NLO predictions together with corresponding uncertainty bands resulting from scale variations. The lower panel displays the differential $\mathcal{K}$ factor together with the uncertainty band (red band). Also shown is the relative scale uncertainty of the LO cross section (blue band). Renormalisation and factorisation scales are set to the common value $\mu_{R}=\mu_{F}=\mu_{0}$ where $\mu_{0}=m_{t}, \mu_{0}=E_{T} / 2$ and $\mu_{0}=H_{T} / 2$. The CT14 PDF sets are employed.

physics models, where top quark pairs are produced through the exchange of new heavy particles, e.g. heavy Higgs boson(s) from supersymmetric extensions of the SM, a heavy neutral resonance $Z^{\prime}$ or Kaluza-Klein excitations of gluons. Any deviation from the SM shape and normalisation in these observables could signal the presence of new physics. Thus, they need to be described as precisely as possible without any approximations. In figure 7 we can observe that for a fixed scale, $\mu_{0}=m_{t}$, the NLO corrections to the topquark transverse momentum distribution do not simply rescale the LO shapes, but induce distortions of the order of $80 \%$. Clearly, substantial, of the order of $60 \%$, negative NLO corrections affect the high $p_{T, t}$ region. We also note that the NLO error bands do not fit within the LO ones as one would expect from a well-behaved perturbative expansion. Thus, the fixed scale choice does not ensure a stable shape when going from LO to NLO for this observable. Through the implementation of a dynamical scale, large discrepancies between the shapes of these distributions at NLO and LO have disappeared. Even though the resulting differential $\mathcal{K}$-factor is not flat the NLO QCD corrections are substantially decreased in the tails, which is mainly due to large changes in the LO distributions in that region. To be more specific, at the central value of the scale, high $p_{T}$ tails received negative but tiny (3\%) NLO corrections. Overall distortions are around $25 \%$ independently of the dynamical scale choice. In general the LO curve is much more sensitive to the variation of the scale and will change more rapidly than the NLO one. In addition, one can observe 
that the NLO error bands as calculated through scale variation nicely fit within the LO error bands. Also in the case of the differential cross section $\mu_{0}=H_{T} / 2$ provides the smallest theoretical uncertainties in the whole plotted range. In figure 8 we plot $p_{T, t}$ again, this time, however, LO and NLO spectra are given separately only for the central value of the given scale. On the other hand, in the lower panel a ratio of both dynamical scale choices to $\mu_{0}=m_{t}$ is displayed. We can notice that at LO already around $100 \mathrm{GeV}$ curves described by dynamical scales, $\mu_{0}=E_{T} / 2$ and $\mu_{0}=H_{T} / 2$ vary substantially from the one given by $\mu_{0}=m_{t}$. The latter yields a much harder spectrum. At NLO, the difference between the fixed and the dynamical scale is smaller, as it should be, because of the reduced dependence of NLO results on the renormalisation and factorisation scales. Up to $300 \mathrm{GeV}$ predictions for all three scales are in agreement. Above $300 \mathrm{GeV}$, however, $\mu_{0}=m_{t}$ gives a softer spectrum as compared to $\mu_{0}=E_{T} / 2$ and $\mu_{0}=H_{T} / 2$. Given the better performance in terms of perturbative stability, we believe that the dynamical scales are more appropriate to model the high- $p_{T}$ tails. For the rapidity distribution of the top quark, shown in figure 9, we observe a different pattern. QCD corrections for $\mu_{0}=m_{t}$ are negative, moderate (below 12\%) and quite stable in the whole rapidity range. This can be easily understood since $y_{t}$ is a dimensionless observable, that receives contributions from all scales, most notably from those that are sensitive to the threshold for the $t \bar{t}$ production. Dynamical scales do not alter this behaviour but rather affect only the normalisation of the LO prediction, which can be observed in figure 10. As a consequence for $\mu_{0}=E_{T} / 2$ and $\mu_{0}=H_{T} / 2$ positive, moderate $(10 \%-15 \%)$ and quite stable NLO corrections are obtained. Also for this observable $\mu_{0}=H_{T} / 2$ provides the smallest theoretical uncertainties. Finally, for the invariant mass of the $t \bar{t}$ pair we expect a similar behaviour as in case of $p_{T, t}$ due to dimensionful nature of the observable. Indeed, we can see in figures 11 and 12 that our conclusions remain qualitatively unaffected. The large negative QCD corrections, of the order $40 \%-60 \%$, which characterize the $\mathrm{TeV}$ range in the case of $\mu_{0}=m_{t}$ are sensibly reduced to about $5 \%$ using $\mu_{0}=E_{T} / 2$ or $\mu_{0}=H_{T} / 2$. The latter two choices are also legitimate options to describe correctly the NLO spectrum in the $M_{t \bar{t}} \in(0.7-1.5) \mathrm{TeV}$ range as can be observed in figure 12 .

In the next step we shall present observables that are constructed from visible topquark decay products, i.e. light- and bottom-jets, charged leptons and missing transverse momentum. Therefore, they are directly accessible without any need for the convoluted reconstruction. We start with the transverse momentum and rapidity of the hardest lightjet, depicted in figures 13, 14 and 15. The kinematics of the hardest light-jet is particularly important when additional jet activity in $t \bar{t}$ events is studied. It is greatly sensitive to higher-order perturbative QCD effects and several theoretical approaches are available to model it. The detailed description of $p_{T, j_{1}}$ and $y_{j_{1}}$ can be used for example to test various parton-shower models and different methods for matching fixed-order QCD calculations with parton shower frameworks. We note that for $p_{T, j_{1}}$ the fixed scale choice yields negative corrections within the 4\%-40\% range. Once dynamical scales are employed positive NLO QCD corrections below $20 \%$ are obtained. Moreover, at NLO up to $400 \mathrm{GeV}$ all scale choices can be applied to describe the $p_{T}$ spectrum of the hardest light-jet. A similar reduction can be observed for the rapidity distribution where negative corrections of the order of $10 \%$ 

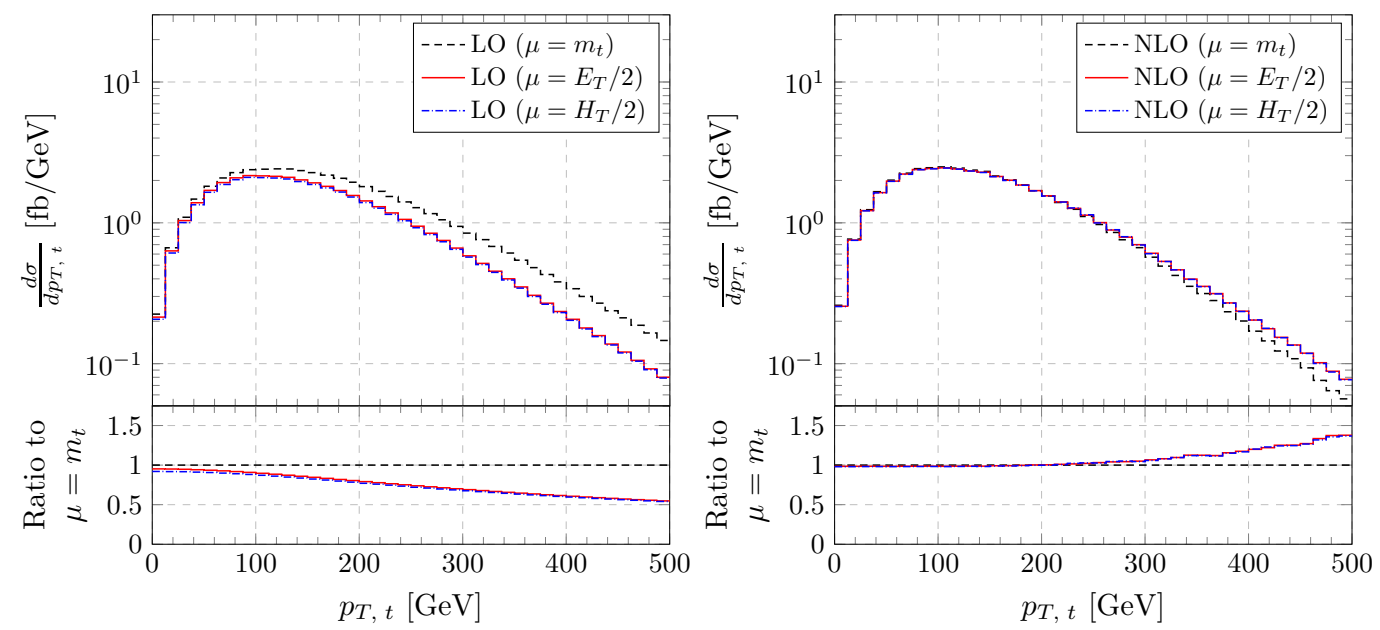

Figure 8. Averaged differential cross section distributions as a function of the transverse momentum of the top quark at LO (left panel) and at NLO (right panel). Results are given for the $p p \rightarrow e^{+} \nu_{e} \mu^{-} \bar{\nu}_{\mu} b \bar{b} j+X$ process at the LHC run II with $\sqrt{s}=13 \mathrm{TeV}$ with $\mu_{R}=\mu_{F}=\mu_{0}$ where $\mu_{0}=m_{t}, \mu_{0}=E_{T} / 2$ and $\mu_{0}=H_{T} / 2$. The lower panel displays a ratio to the prediction with $\mu_{0}=m_{t}$. The CT14 PDF sets are employed.

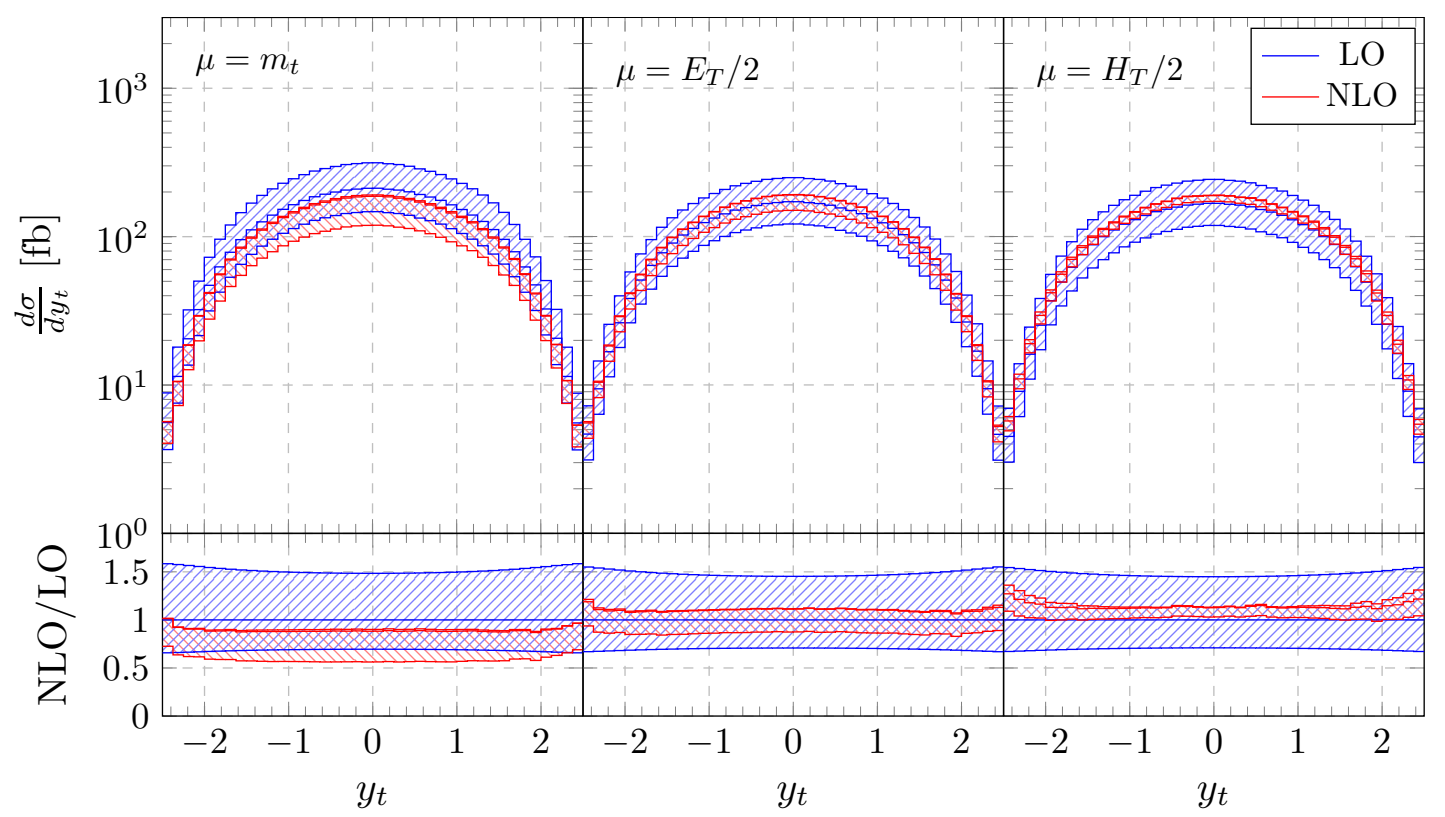

Figure 9. Averaged differential cross section distributions as a function of the rapidity of the top quark for the $p p \rightarrow e^{+} \nu_{e} \mu^{-} \bar{\nu}_{\mu} b \bar{b} j+X$ process at the LHC run II with $\sqrt{s}=13 \mathrm{TeV}$.

for $\mu_{0}=m_{t}$ are replaced by positive corrections of the similar size for both $\mu_{0}=E_{T} / 2$ and $\mu_{0}=H_{T} / 2$. Again theoretical uncertainties as obtained with the $\mu_{0}=H_{T} / 2$ are the smallest for both observables. We have drawn qualitatively similar conclusions for bottom-jet and charged lepton kinematics that are displayed for completeness in figures 16 and 17 as well as in figures 18-21. For the averaged $p_{T}$ distributions of the bottom-jet and 

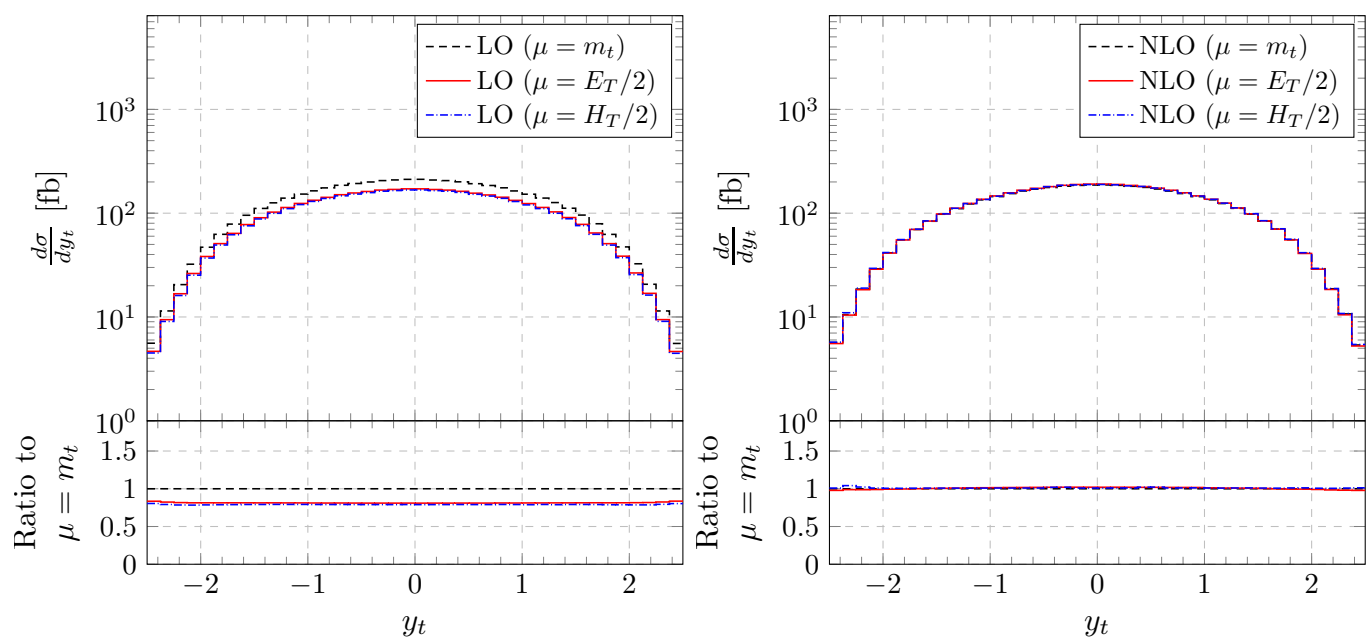

Figure 10. Averaged differential cross section distributions as a function of the rapidity of the top quark at LO (left panel) and at NLO (right panel) for the $p p \rightarrow e^{+} \nu_{e} \mu^{-} \bar{\nu}_{\mu} b \bar{b} j+X$ process at the LHC run II with $\sqrt{s}=13 \mathrm{TeV}$.

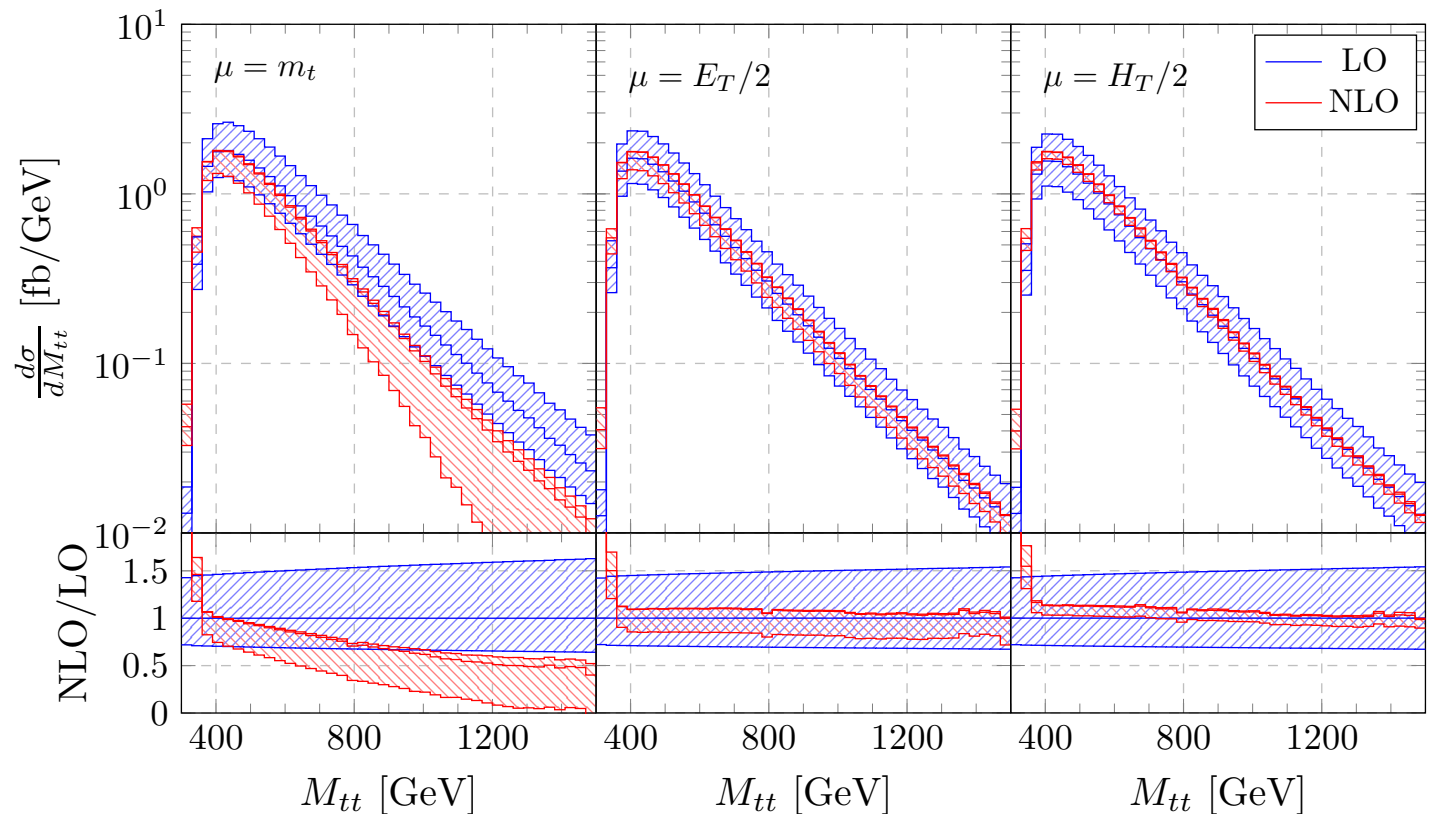

Figure 11. Differential cross section distribution as a function of the invariant mass of the $t \bar{t}$ system for the $p p \rightarrow e^{+} \nu_{e} \mu^{-} \bar{\nu}_{\mu} b \bar{b} j+X$ process at the LHC run II with $\sqrt{s}=13 \mathrm{TeV}$.

charged leptons, we observe even more pronounced NLO corrections, reaching $55 \%$ and $65 \%$ respectively in the tails. They have been replaced by positive corrections below $20 \%$ when $\mu_{0}=E_{T} / 2$ or $\mu_{0}=H_{T} / 2$ has been used instead. For both rapidity distributions negative corrections of the order of $10 \%$ with $\mu_{0}=m_{t}$ have been substituted by positive ones, which are of the same order for $\mu_{0}=E_{T} / 2$ and below $20 \%$ with the $\mu_{0}=H_{T} / 2$ scale choice. Additionally, already around $250 \mathrm{GeV}$ and $150 \mathrm{GeV}$ respectively for $p_{T, b}$ and $p_{T, \ell}$, NLO distributions are properly described only by the dynamical scale choice, either 

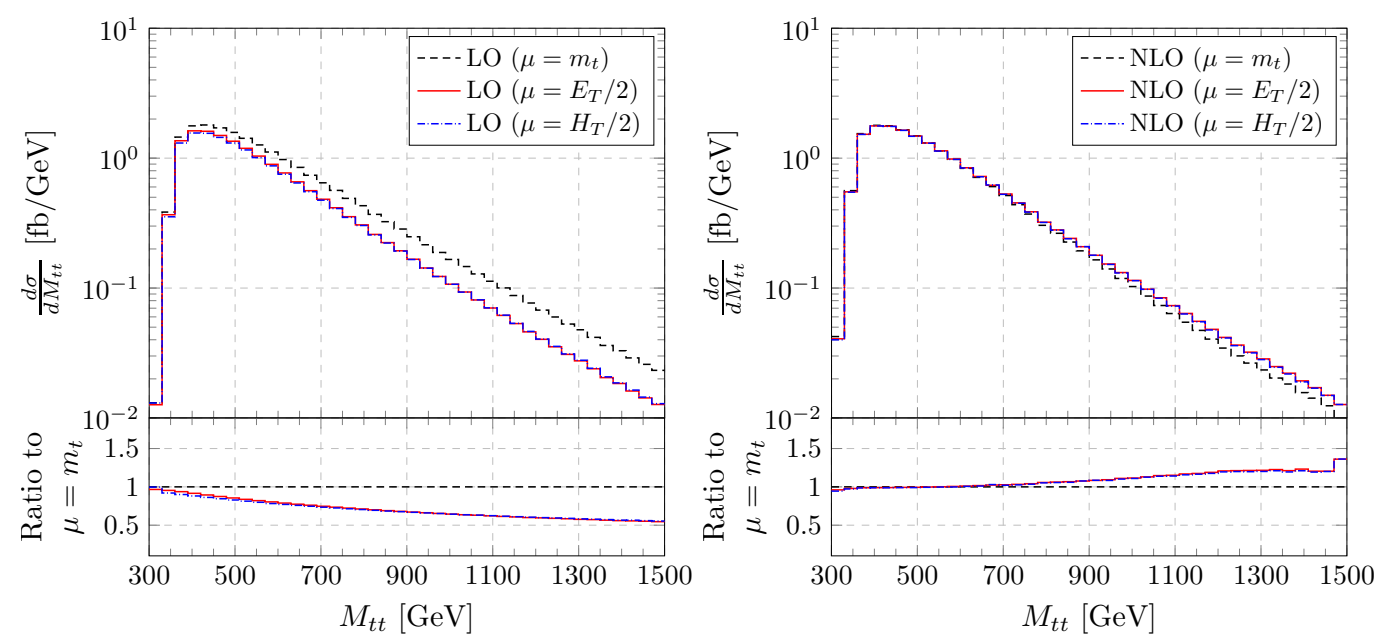

Figure 12. Differential cross section distributions as a function of the invariant mass of the $t \bar{t}$ pair at LO (left panel) and at NLO (right panel) for the $p p \rightarrow e^{+} \nu_{e} \mu^{-} \bar{\nu}_{\mu} b \bar{b} j+X$ process at the LHC run II with $\sqrt{s}=13 \mathrm{TeV}$.

$\mu_{0}=E_{T} / 2$ or $\mu_{0}=H_{T} / 2$. Let us also note here that looking at the rapidity distributions of the light- and the bottom-jet as well as the charged lepton we can observe a very well known fact, namely, that bottom-jets and positrons or muons are distributed centrally in the rapidity, while the light-jet spans a broader range. This information is used for example to develop dedicated cuts to reduce top-quark backgrounds for various signal processes.

Other observables, that are crucial in new physics searches in the dilepton decay channel of the top quark, are the missing transverse momentum, denoted here as $\not p_{T}$, the total transverse momentum of the system, $H_{T}$, and the invariant mass of two charged leptons, $M_{\ell \ell}$. They are presented in figures 22 and 23. Various new physics models postulate the existence of new particles that might decay into a $t \bar{t}$ pair plus other final states. The most prominent example is pair production of top-quark partners decaying to a top-quark pair and a long-lived neutral particle, which escapes undetected. This weakly interacting particle would manifest itself as a large missing energy in the ATLAS and CMS detectors and would lead to the $p p \rightarrow T \bar{T} \rightarrow t \bar{t}+\not p_{T}$ signature, where $T$ generically denotes the top-quark partner. The above signature appears in numerous new physics scenarios, see e.g. [90-93]. Since these three observables constitute a very powerful tool in the BSM physics searches we also plot them separately in figures 24,25 and 26, for the central scale only and for three different scale choices. From the latter plots we can see that only $\mu_{0}=E_{T} / 2$ and $\mu_{0}=H_{T} / 2$ describe these observables properly in the hight $p_{T}$ tails and that both dynamical scales give the same prediction in the whole plotted ranges. However, the former distributions tell us that $\mu_{0}=H_{T} / 2$ grants the smallest theoretical uncertainties for each observable. Overall, for $\not p_{T}$ we obtained large and negative NLO corrections, which reach $50 \%$ around $300 \mathrm{GeV}$ when $\mu_{0}=m_{t}$ is applied. As long as dynamical scales are used instead, these corrections are replaced by positive and moderate ones, which are up to $10 \%$ for $\mu_{0}=E_{T} / 2$ and of the order of $10 \%-20 \%$ for $\mu_{0}=H_{T} / 2$. For the total transverse momentum of the $t \bar{t} j$ system we have noticed a comparable performance. Specifically, around 

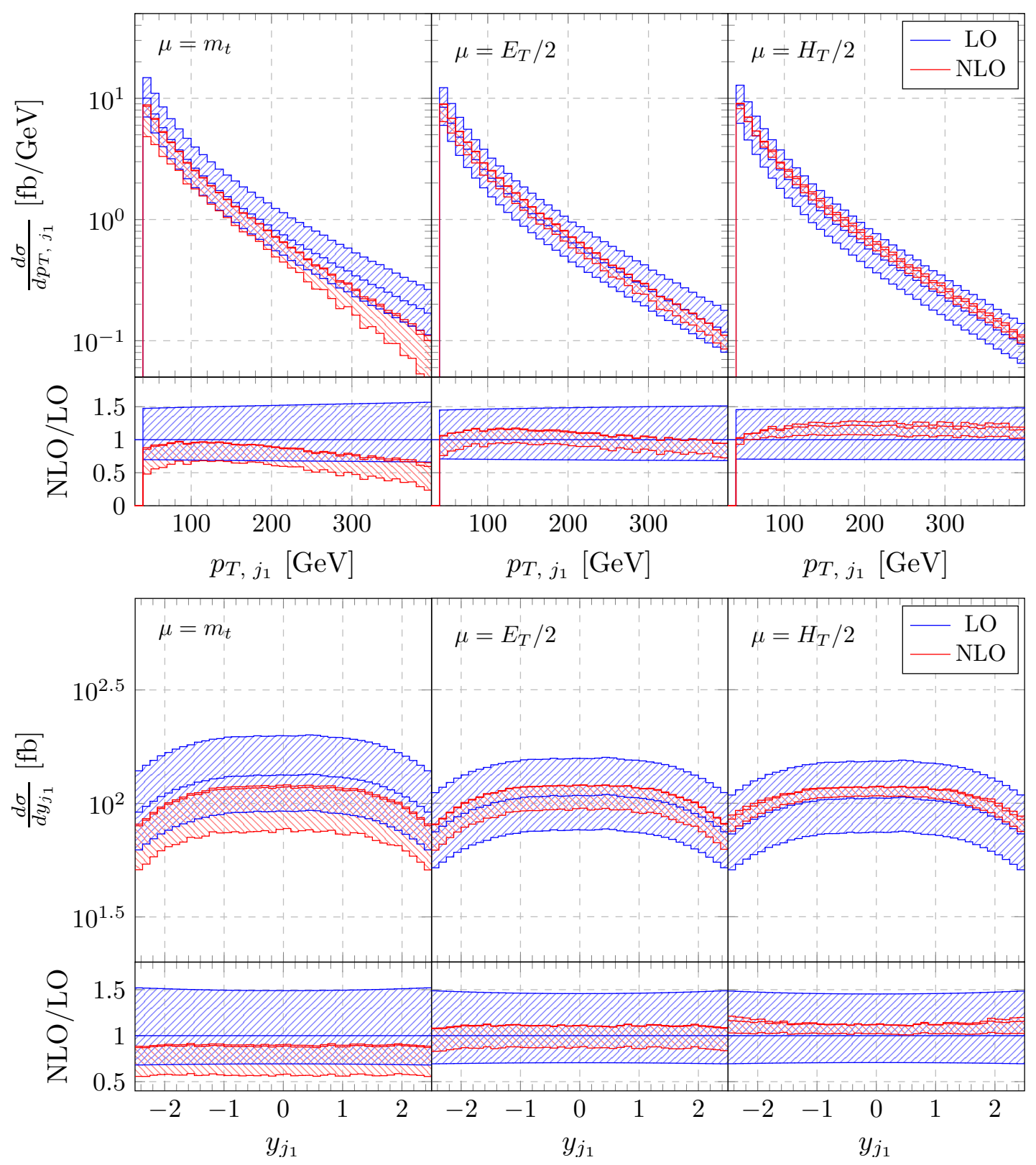

Figure 13. Differential cross section distribution as a function of the transverse momentum and rapidity of the hardest jet for the $p p \rightarrow e^{+} \nu_{e} \mu^{-} \bar{\nu}_{\mu} b \bar{b} j+X$ process at the LHC run II with $\sqrt{s}=13 \mathrm{TeV}$.

$1500 \mathrm{GeV}-70 \%$ corrections at $\mu_{0}=m_{t}$ have been downsized to about $-10 \%$ and $+10 \%$ for $\mu_{0}=E_{T} / 2$ and $\mu_{0}=H_{T} / 2$ respectively. Lastly, for the invariant mass of two charged leptons $-50 \% \mathrm{NLO}$ corrections around $500 \mathrm{GeV}$ for $\mu_{0}=m_{t}$ have been converted to $-1.5 \%$ for $\mu_{0}=E_{T} / 2$ and almost to zero corrections for $\mu_{0}=H_{T} / 2$.

In figure 27 we present the differential cross section as a function of the separation of charged leptons in the rapidity-azimuthal angle plane, $\Delta R_{\ell \ell}=\sqrt{\Delta \phi_{\ell \ell}^{2}+\Delta y_{\ell \ell}^{2}}$, and the azimuthal angle between the charged leptons $\Delta \phi_{\ell \ell}=\left|\phi_{\ell_{1}}-\phi_{\ell_{2}}\right|$. They are measured 

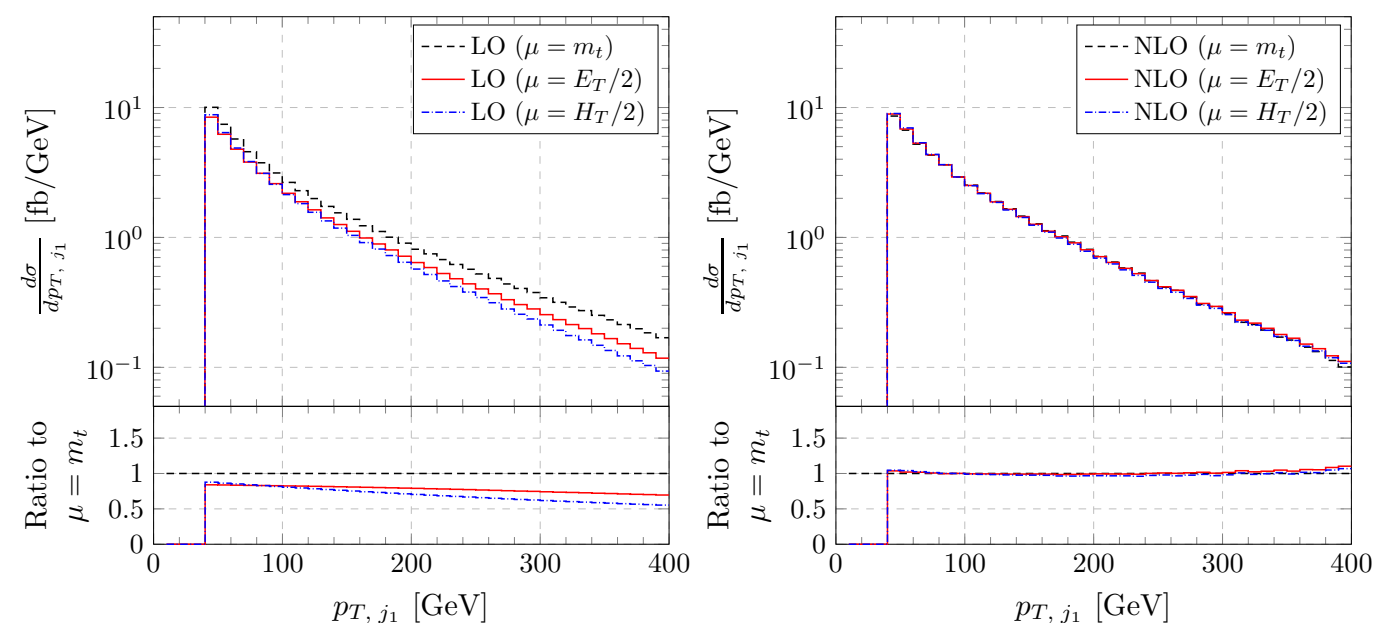

Figure 14. Differential cross section distributions as a function of the transverse momentum of the first hardest jet at LO (left panel) and at NLO (right panel) for the $p p \rightarrow e^{+} \nu_{e} \mu^{-} \bar{\nu}_{\mu} b \bar{b} j+X$ process at the LHC run II with $\sqrt{s}=13 \mathrm{TeV}$.
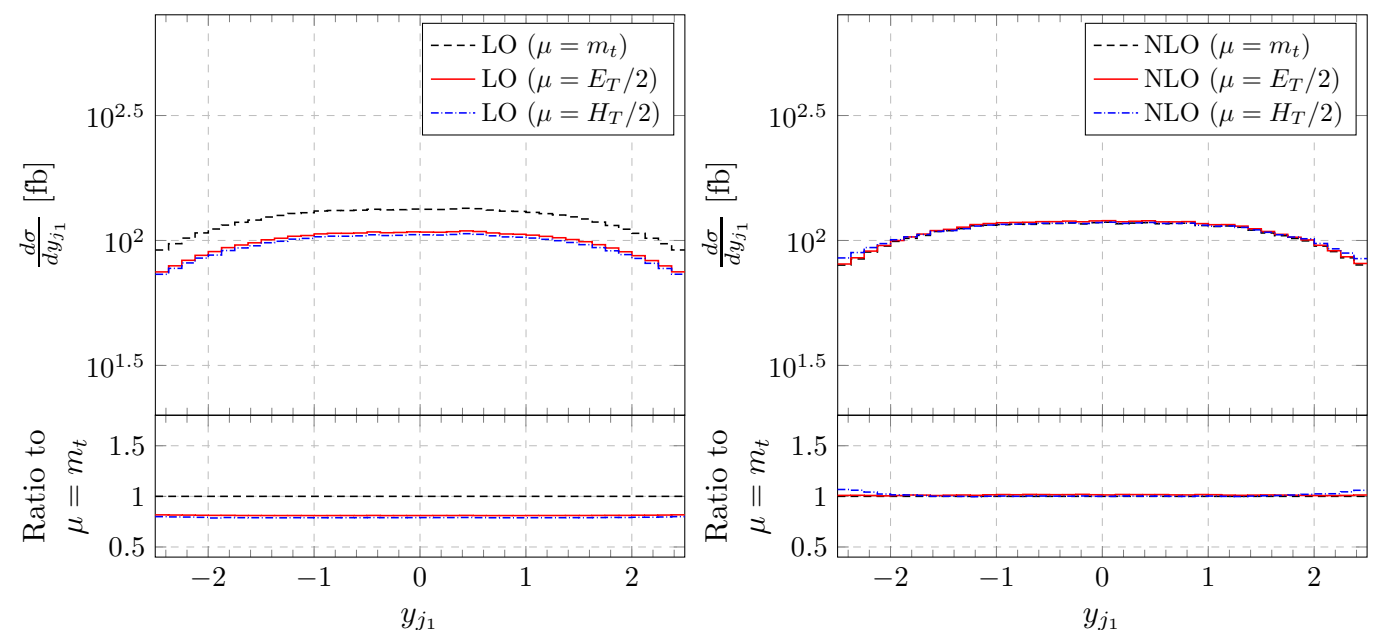

Figure 15. Differential cross section distributions as a function of the rapidity of the first hardest jet at LO (left panel) and at NLO (right panel) for the $p p \rightarrow e^{+} \nu_{e} \mu^{-} \bar{\nu}_{\mu} b \bar{b} j+X$ process at the LHC run II with $\sqrt{s}=13 \mathrm{TeV}$.

very precisely at the LHC by both ATLAS and CMS collaborations and do not require the reconstruction of the top quarks. In general, angular distributions of charged leptons are of huge importance since they reflect spin correlations of the top-quark pair. Because of its large mass, the top quark is extremely short-lived. As a result, top quarks do not have time to form hadrons before they decay. Thus, the spin of the top-quark pair at production is transferred to the decay products and can be measured directly via their angular distributions [94]. Many models of new physics predict vastly different spin correlations while keeping similar production cross sections, an example being the production of heavy spin-zero states with undefined CP parity and mass below $400 \mathrm{GeV}$ that are resonantly produced in the $t \bar{t}$ channel [95]. Therefore, in practice top-quark 

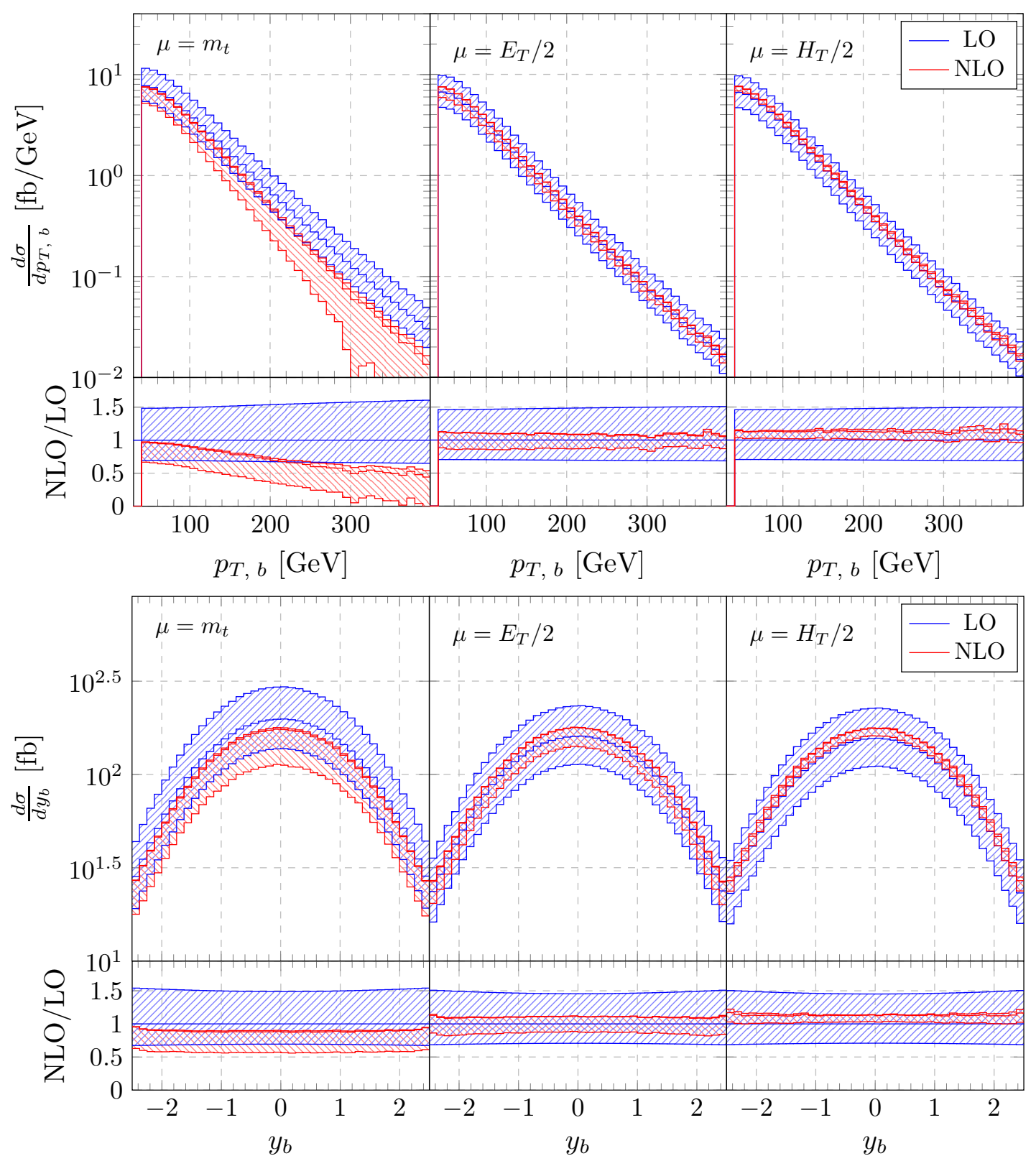

Figure 16. Averaged differential cross section distribution as a function of the transverse momentum and rapidity of the bottom-jet for the $p p \rightarrow e^{+} \nu_{e} \mu^{-} \bar{\nu}_{\mu} b \bar{b} j+X$ process at the LHC run II with $\sqrt{s}=13 \mathrm{TeV}$.

pair spin correlations can be used by experimental collaborations at the LHC to provide a handle on the determination of the nature of the new particle that decays as $p p \rightarrow h_{\text {new }} \rightarrow t \bar{t}+X \rightarrow W^{+} W^{-} b \bar{b}+X \rightarrow \ell^{+} \ell^{-} \nu_{\ell} \bar{\nu}_{\ell} b \bar{b}+X$, where $h_{\text {new }}$ is the heavy spin-zero state. Even though $\Delta R_{\ell \ell}$ and $\Delta \phi_{\ell \ell}$ are dimensionless observables, we observe in figure 27 that they receive quite large NLO corrections with $\mu_{0}=m_{t}$, which vary within the plotted ranges. To be more specific for $\Delta R_{\ell \ell}$ we obtained a variation between $+5 \%$ and $-25 \%$, for $\Delta \phi_{\ell \ell}$ we reached a change between $+10 \%$ and $-30 \%$. On the other hand, for our 

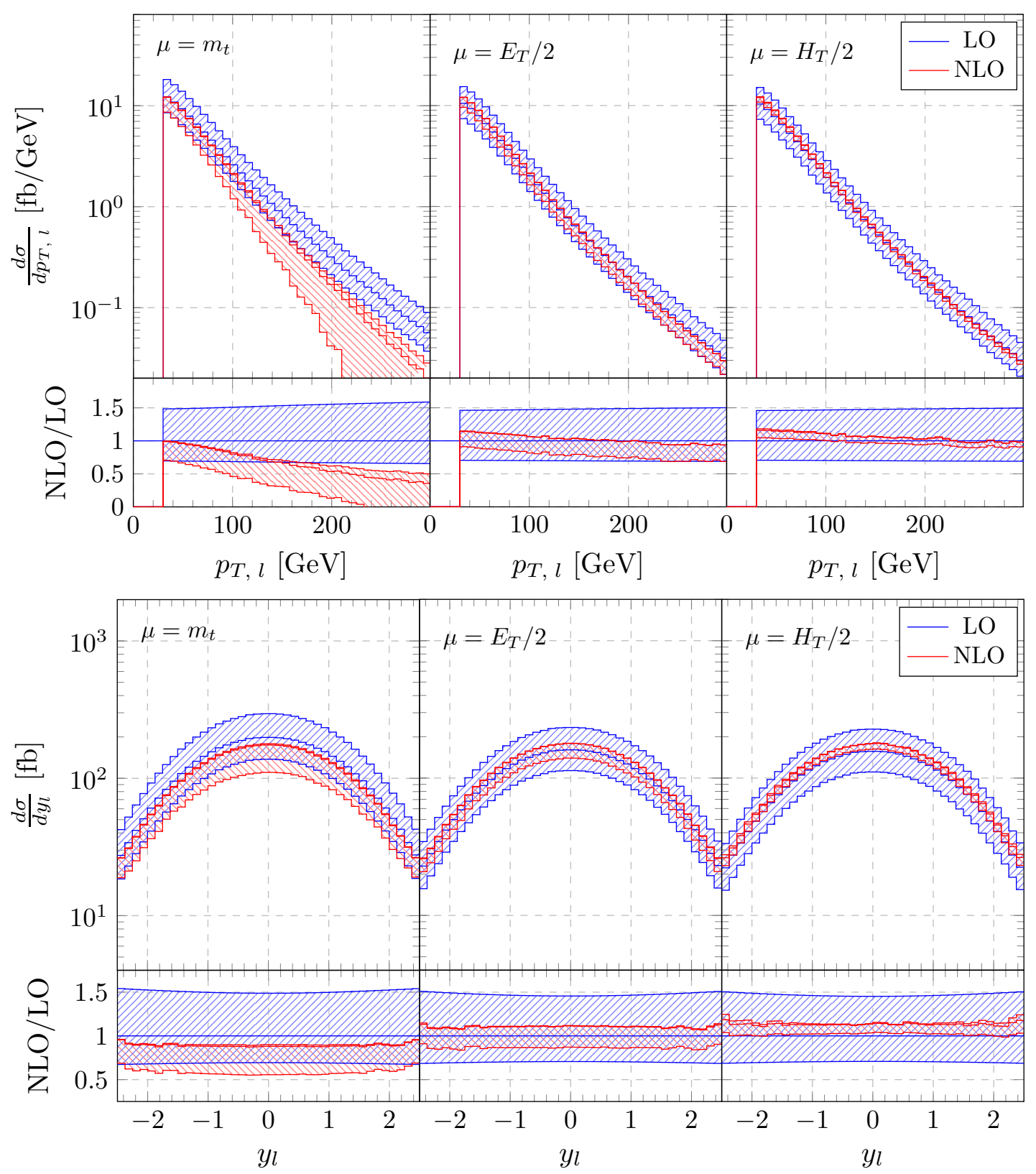

Figure 17. Averaged differential cross section distribution as a function of the transverse momentum and rapidity of the charged lepton for the $p p \rightarrow e^{+} \nu_{e} \mu^{-} \bar{\nu}_{\mu} b \bar{b} j+X$ process at the LHC run II with $\sqrt{s}=13 \mathrm{TeV}$.

best scale choice, $\mu_{0}=H_{T} / 2$, positive corrections in the whole shown range are realised for both observables. Specifically, we have noticed $5 \%-25 \%$ corrections for $\Delta R_{\ell \ell}$ and $2 \%-30 \%$ for $\Delta \phi_{\ell \ell}$. Similar results have been reached with $\mu_{0}=E_{T} / 2$. From figures 28 and 29 we can further see that at NLO dependence on the scale choice is practically non existing in both cases, unlike at LO where the $\mu_{0}=m_{t}$ choice always predicts higher spectra.

The last observables that we present are the invariant mass of the positron and bottomjet, $M_{b e^{+}}$and the mass of the reconstructed top quark, $M_{t}$. The latter is given only in 

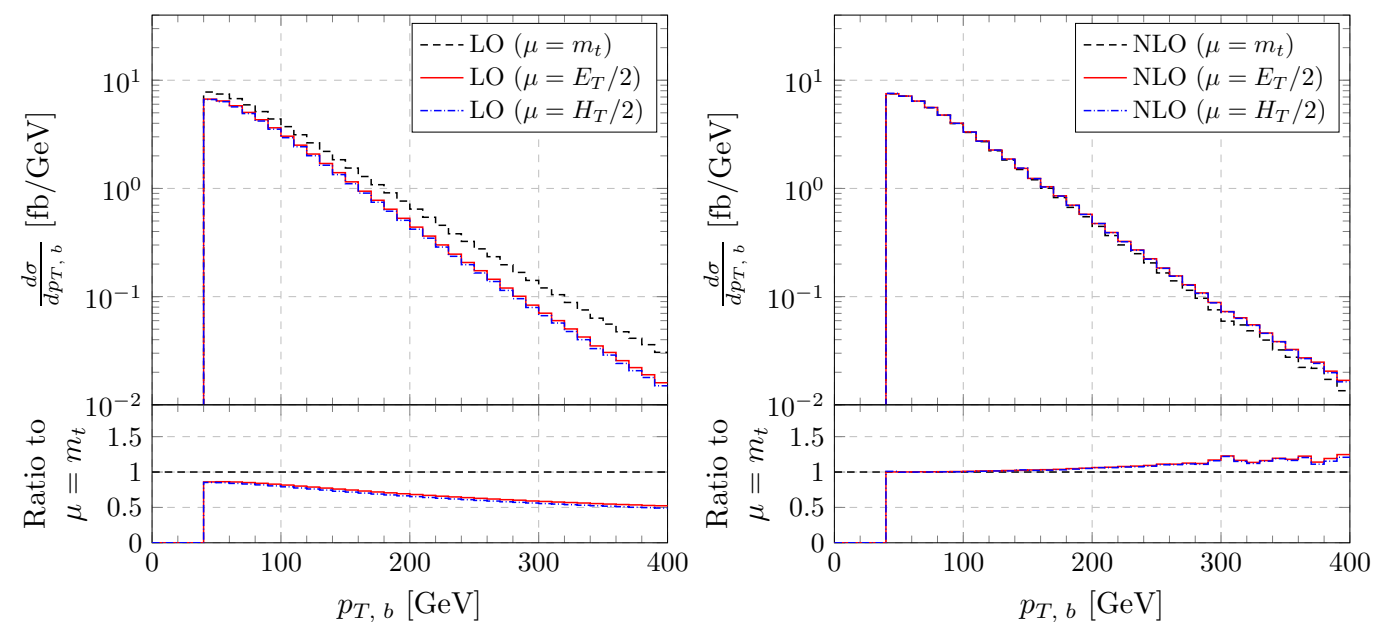

Figure 18. Averaged differential cross section distributions as a function of the transverse momentum of the bottom-jet at LO (left panel) and at NLO (right panel) for the $p p \rightarrow e^{+} \nu_{e} \mu^{-} \bar{\nu}_{\mu} b \bar{b} j+X$ process at the LHC run II with $\sqrt{s}=13 \mathrm{TeV}$.
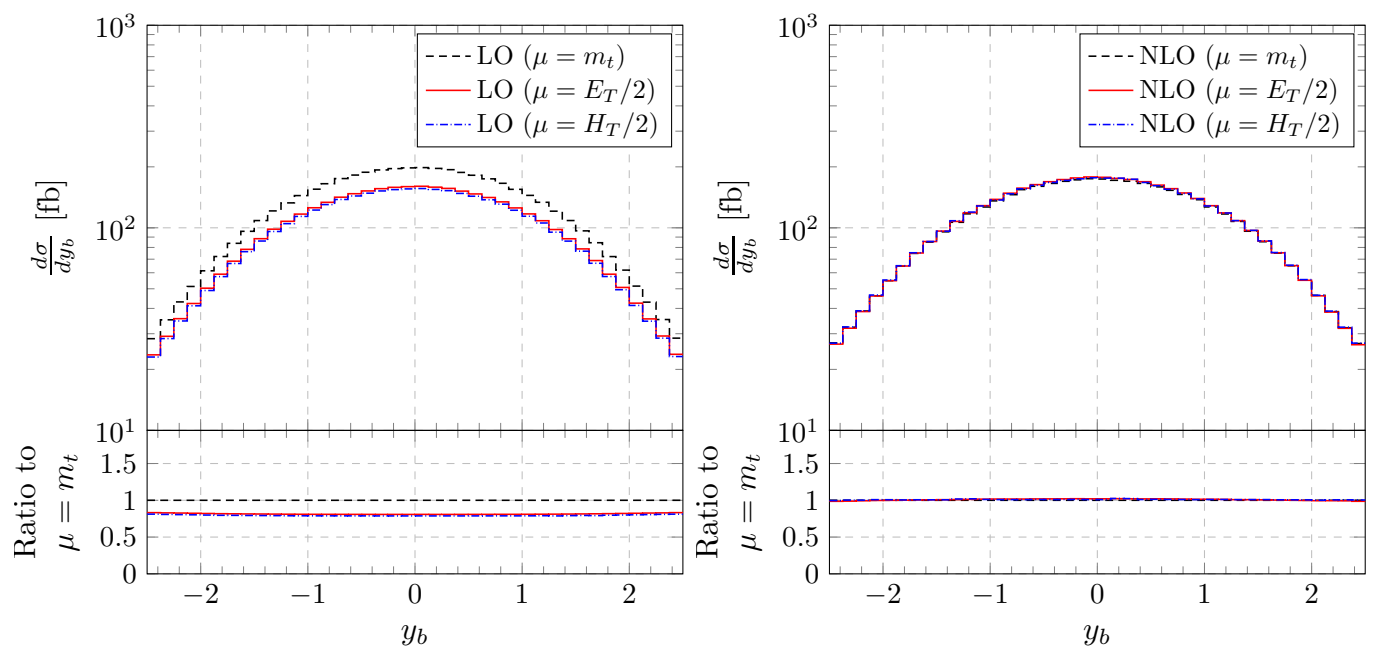

Figure 19. Averaged differential cross section distributions as a function of the rapidity of the bottom-jet at LO (left panel) and at NLO (right panel) for the $p p \rightarrow e^{+} \nu_{e} \mu^{-} \bar{\nu}_{\mu} b \bar{b} j+X$ process at the LHC run II with $\sqrt{s}=13 \mathrm{TeV}$.

the vicinity of the resonance. They are both plotted in figures 30,31 and 32. These observables are crucial for the top-quark mass extraction. In the case of $M_{b e^{+}}$one cannot determine, which b-jet should be paired with the positron. To increase the probability that both final states come from the decay cascade initiated by the same top quark we select the $b e^{+}$pair, that returns the smallest invariant mass [96]. Thus, $M_{b e^{+}}$is defined as $M_{b e^{+}}=\min \left\{\sqrt{\left(p_{b_{1}}+p_{e^{+}}\right)^{2}}, \sqrt{\left(p_{b_{2}}+p_{e^{+}}\right)^{2}}\right\}$ and contains a kinematic endpoint that can be derived from the on-shell top-quark decay into $t \rightarrow W^{+} b \rightarrow e^{+} \nu_{e} b$. Neglecting the masses of all decay products we can write

$$
m_{t}^{2}=p_{t}^{2}=m_{W}^{2}+2 p_{b} p_{e^{+}}+2 p_{b} p_{\nu_{e}} .
$$



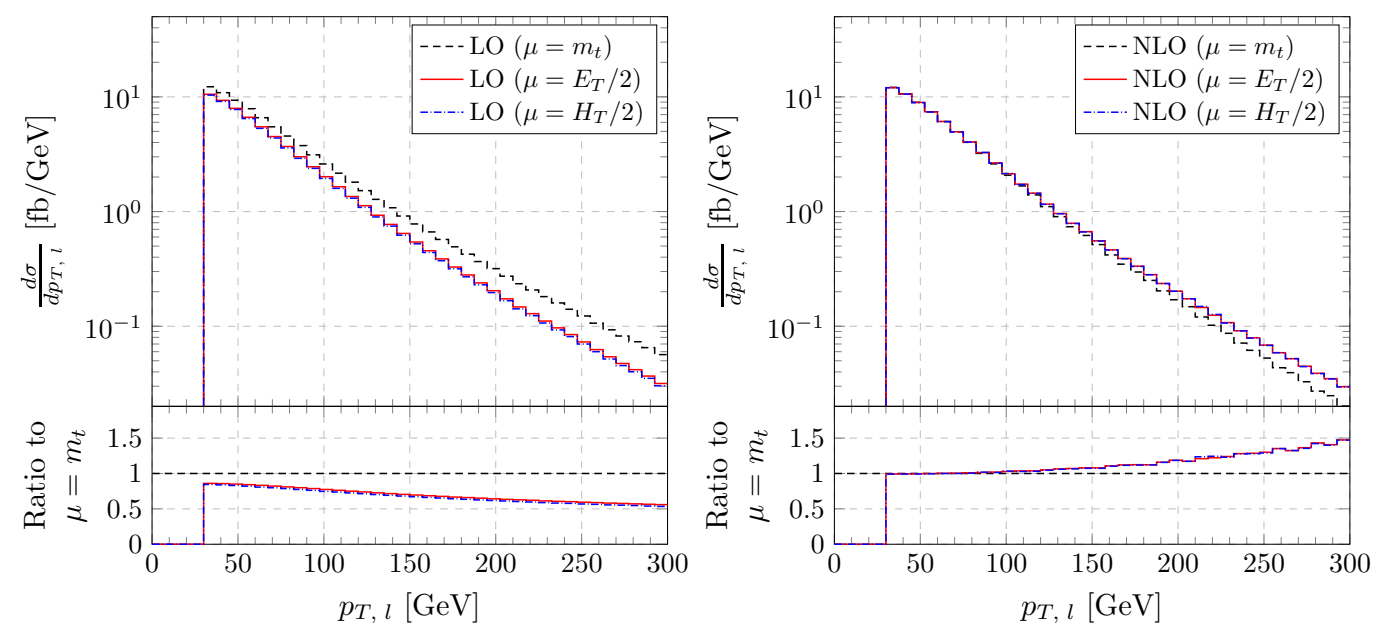

Figure 20. Averaged differential cross section distributions as a function of the transverse momentum of the charged lepton at LO (left panel) and at NLO (right panel) for the $p p \rightarrow e^{+} \nu_{e} \mu^{-} \bar{\nu}_{\mu} b \bar{b} j+X$ process at the LHC run II with $\sqrt{s}=13 \mathrm{TeV}$.
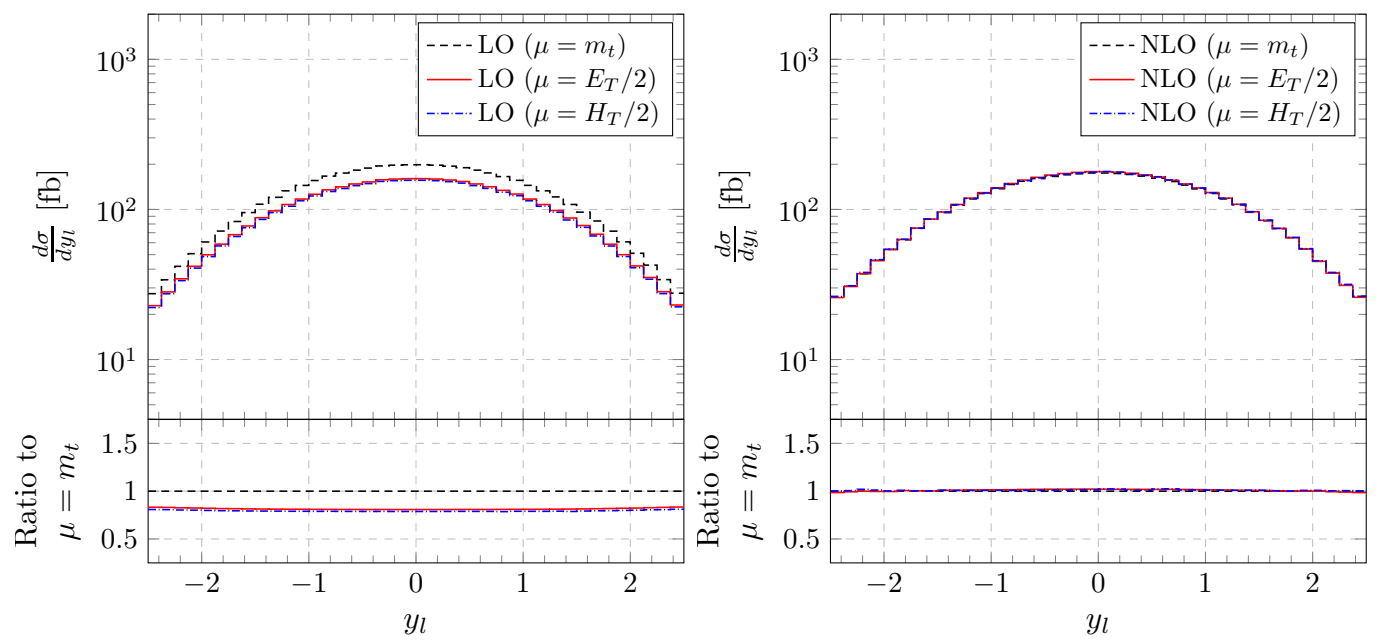

Figure 21. Averaged differential cross section distributions as a function of the rapidity of the charged lepton at LO (left panel) and at NLO (right panel) for the $p p \rightarrow e^{+} \nu_{e} \mu^{-} \bar{\nu}_{\mu} b \bar{b} j+X$ process at the LHC run II with $\sqrt{s}=13 \mathrm{TeV}$.

As a result $M_{b e^{+}}^{2}=2 p_{b} p_{e^{+}} \leq m_{t}^{2}-m_{W}^{2}$. At lowest order when both top quarks and $W$ gauge bosons are treated as on-shell particles there is a strict kinematic limit for the invariant mass of the bottom quark and the positron given by

$$
M_{b e^{+}}^{\max }=\sqrt{m_{t}^{2}-m_{W}^{2}} \approx 153 \mathrm{GeV} .
$$

For off-shell top quarks this kinematic limit is smeared, also additional NLO radiation affects this region, nevertheless there is a sharp fall of the cross section in the fixed order prediction. The two bottom-jets stemming from each $t \bar{t}$ decay give rise to a matching ambiguity. Pairings in which the bottom-jet and positron emerge from different top quarks do not necessarily obey the upper bound $M_{b e^{+}}^{\max }$ and, thus, do not have a clean kinematic 


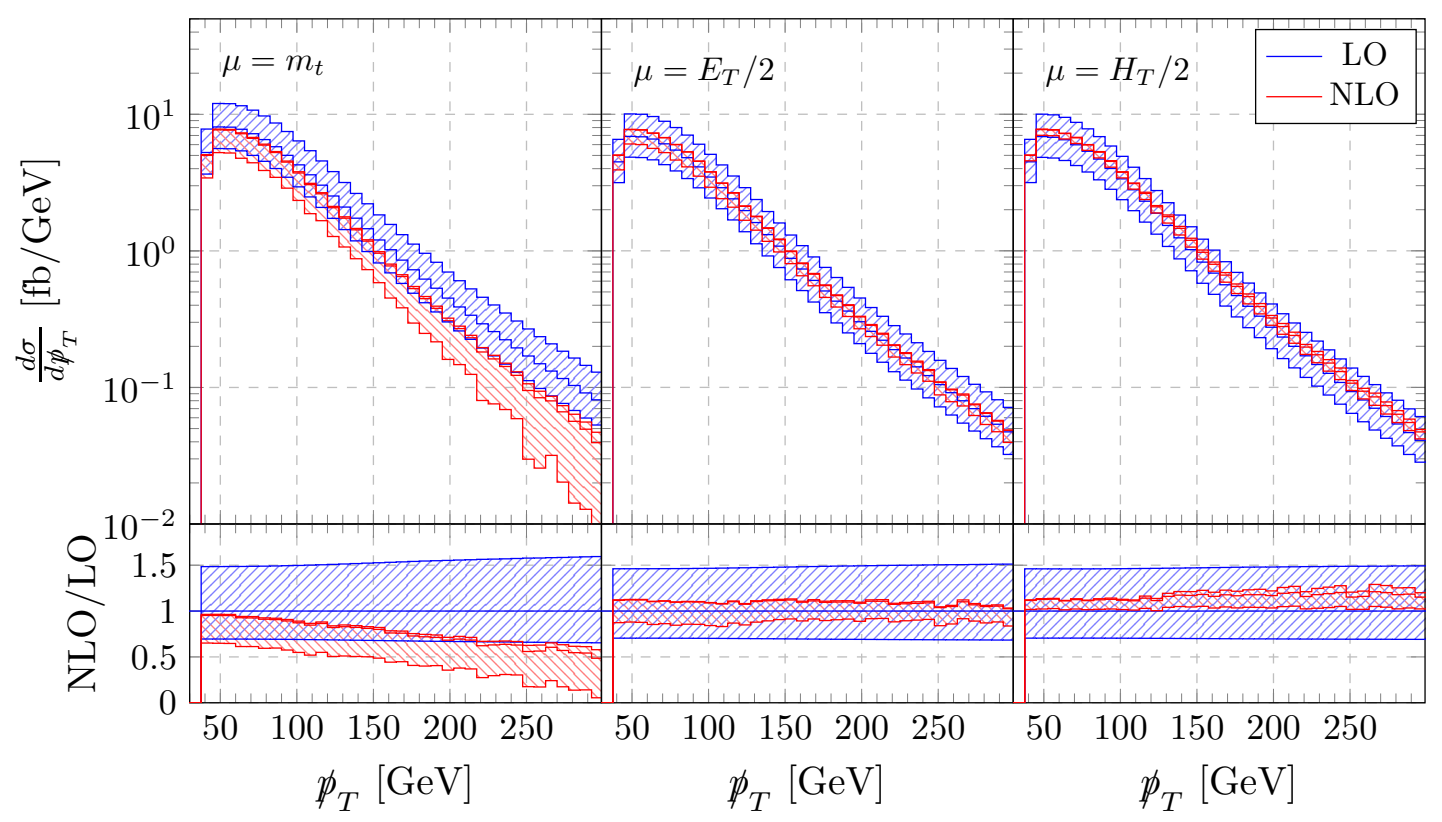

Figure 22. Differential cross section distribution as a function of the missing transverse momentum for the $p p \rightarrow e^{+} \nu_{e} \mu^{-} \bar{\nu}_{\mu} b \bar{b} j+X$ process at the LHC run II with $\sqrt{s}=13 \mathrm{TeV}$.

endpoint. Although a priori it is impossible to distinguish between correct and incorrect pairing, the easiest solution is to select the smallest $M_{b e^{+}}$value in each event as we have done. In this fashion the kinematic endpoint of the distribution is always preserved simply because $M_{b e^{+}} \leq M_{b e^{+}}^{\text {correct }}$. Strong sensitivity of the kinematic endpoint to $m_{t}$ causes this distribution to be extremely useful for the top-quark mass extraction. In the same manner the mass of the reconstructed top quark, defined as $M_{t}=M_{b e^{+} \nu_{e}}=\sqrt{p_{t}^{2}}$ is susceptible to the modelling of the top-quark decays. Off-shell effects and additional gluon radiation further smear the peak resulting from the NWA. NLO QCD corrections affect both distributions greatly. For $M_{b e^{+}}$above $150 \mathrm{GeV}$ corrections above $100 \%$ have been obtained. In more details we have attained NLO QCD corrections of the order of $125 \%, 140 \%$ and $150 \%$ correspondingly for $\mu_{0}=m_{t}, \mu_{0}=E_{T} / 2$ and $\mu_{0}=H_{T} / 2$. In this region, the theoretical uncertainties are also immense independently of the scale used in the calculation. On the other hand, below the kinematical endpoint, moderate negative (positive) corrections in the range 5\%-20\% (5\%-15\% and $10 \%-15 \%)$ are visible for $\mu_{0}=m_{t}\left(\mu_{0}=E_{T} / 2\right.$ and $\mu_{0}=H_{T} / 2$ respectively). The remarkably different behaviour between the two regions can be understood if one considers that the phase space above $M_{b e^{+}}^{\max }$ is populated at LO by genuine off-shell contributions only. As figure 31 suggests, a more proper modeling of the NLO distribution for $M_{b e^{+}}>153 \mathrm{GeV}$ is expected by the use of the dynamical scales. Also, for the $M_{t}$ observable, shown in figure 32, both at LO and NLO, the NLO shape is accurately given only with $\mu_{0}=E_{T} / 2$ and $\mu_{0}=H_{T} / 2$. Moreover, from figure 30 we can read that at the beginning of the spectrum NLO corrections are large up to $120 \%$, $140 \%$ and $145 \%$ for $\mu_{0}=m_{t}, \mu_{0}=E_{T} / 2$ and $\mu_{0}=H_{T} / 2$ respectively. Additionally, large distortions are observed for this observable independently of the scale choice. 

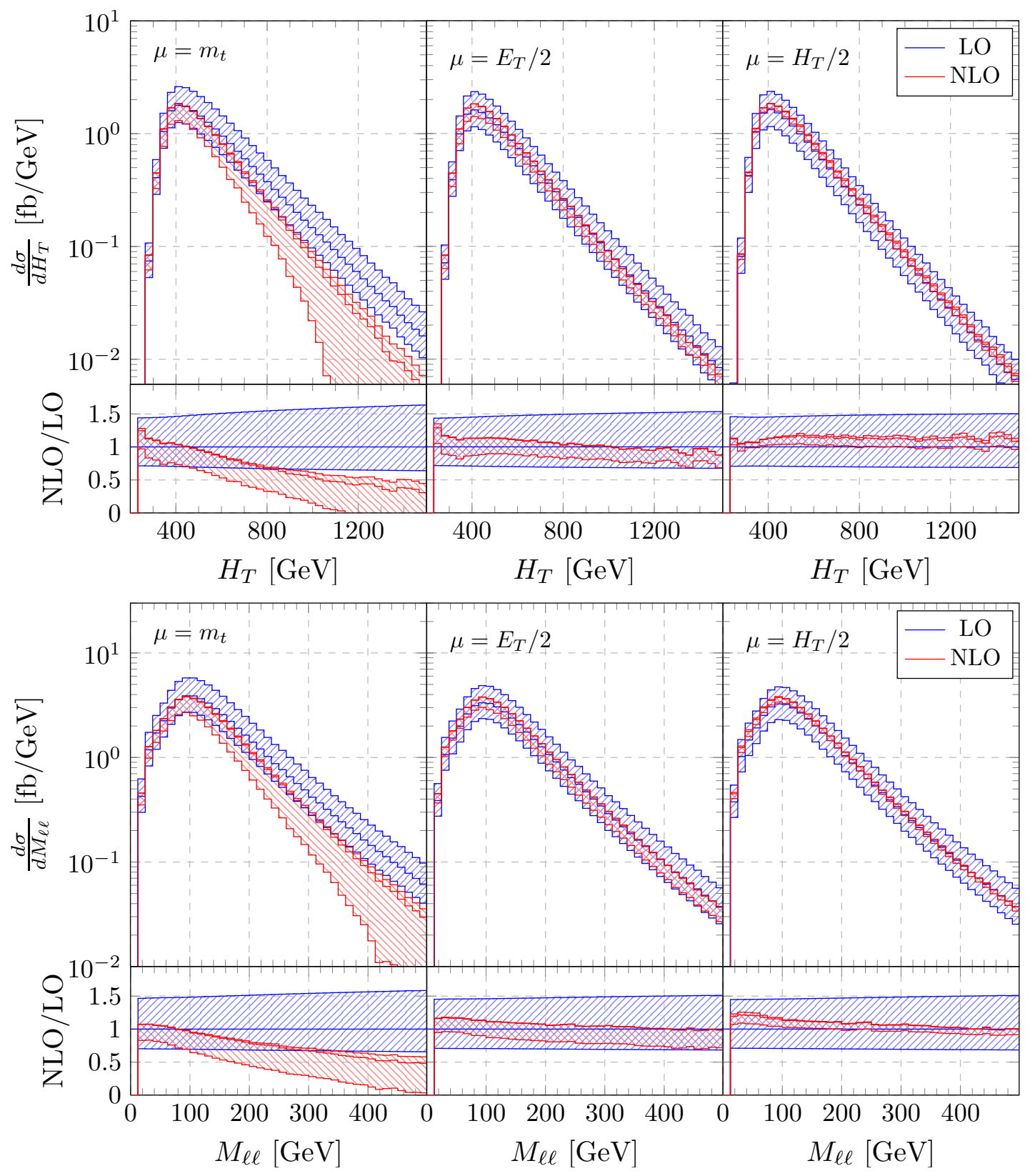

Figure 23. Differential cross section distribution as a function of the total transverse momentum of the system, $H_{T}$ and the invariant mass of two charged leptons for the $p p \rightarrow e^{+} \nu_{e} \mu^{-} \bar{\nu}_{\mu} b \bar{b} j+X$ process at the LHC run II with $\sqrt{s}=13 \mathrm{TeV}$.

To summarise this part, we have studied the size of NLO QCD corrections to numerous differential cross sections. For many observables we have found substantial variations in their magnitude, which depend on the observable itself, the scale choice and the considered phase-space regions. Overall we confirm the validity of the proposed dynamical scales $\mu_{0}=E_{T} / 2$ and $\mu_{0}=H_{T} / 2$, where the latter provides the smallest scale uncertainties. The fixed scale choice $\mu_{0}=m_{t}$, on the contrary, does not prove adequate in our analysis for the modeling of differential cross sections. 

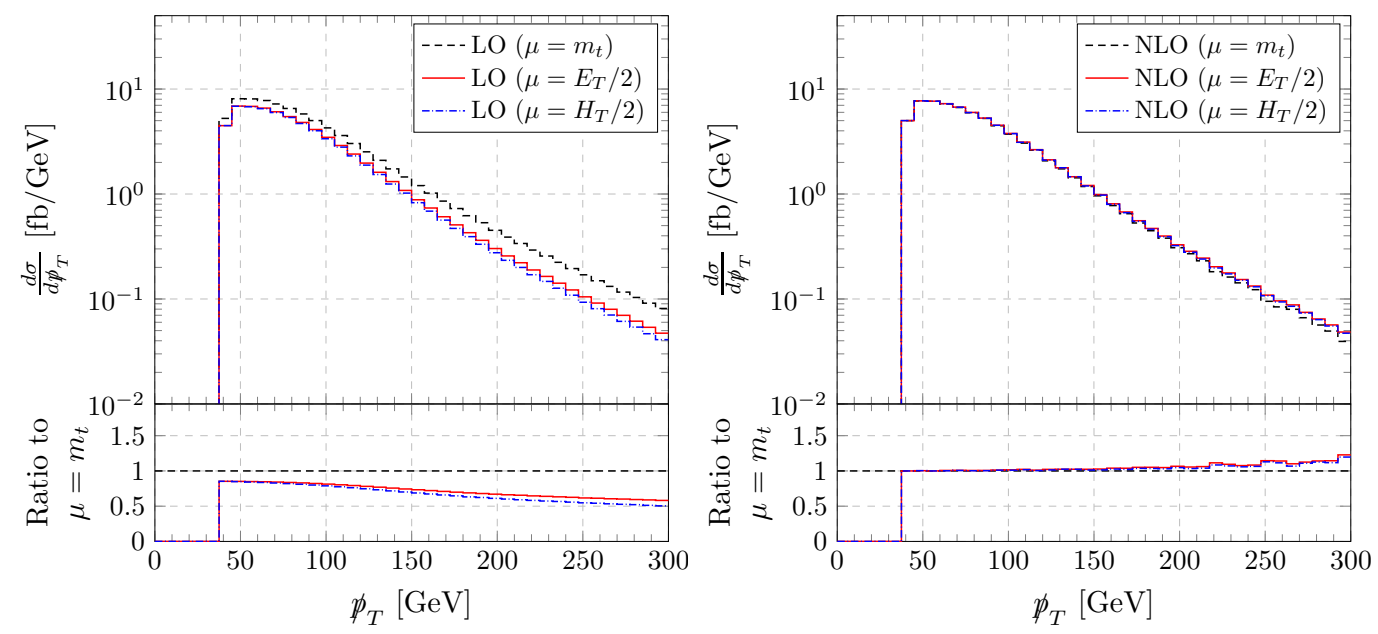

Figure 24. Differential cross section distributions as a function of the missing transverse momentum at LO (left panel) and at NLO (right panel) for the $p p \rightarrow e^{+} \nu_{e} \mu^{-} \bar{\nu}_{\mu} b \bar{b} j+X$ process at the LHC run II with $\sqrt{s}=13 \mathrm{TeV}$.
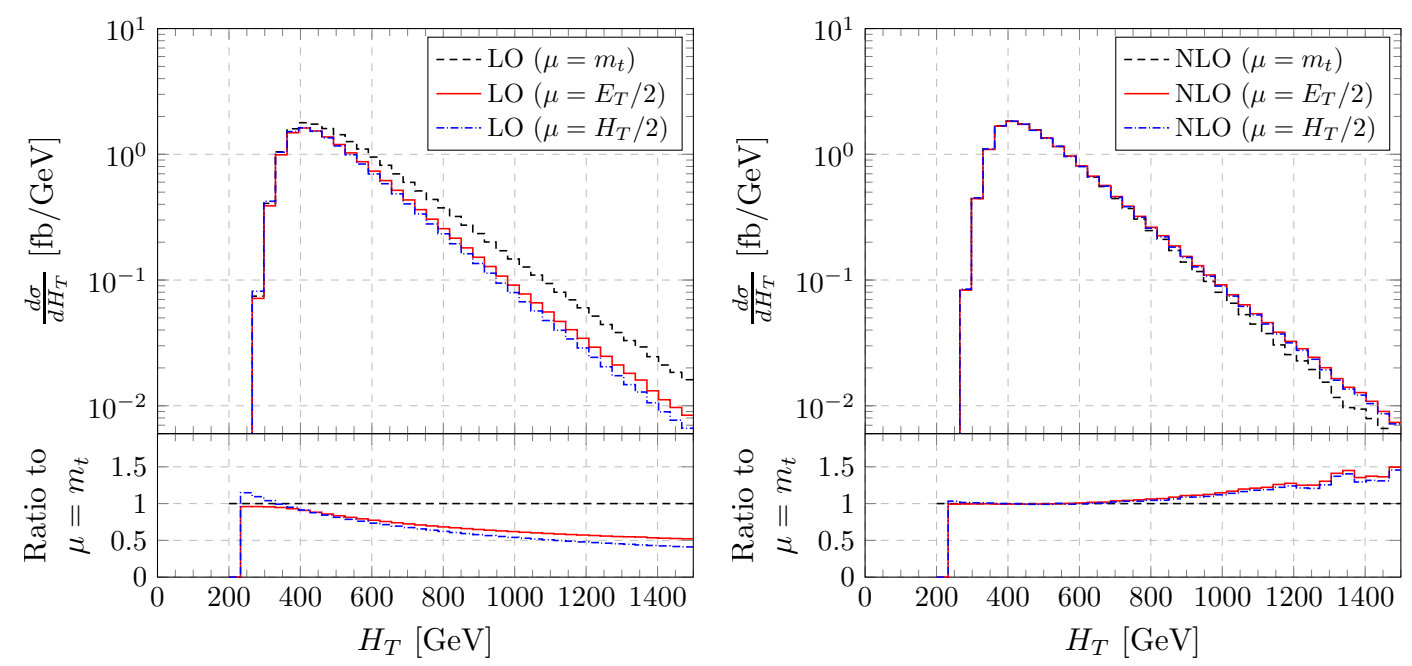

Figure 25. Differential cross section distributions as a function of the total transverse momentum of the system, $H_{T}$, at LO (left panel) and at NLO (right panel) for the $p p \rightarrow e^{+} \nu_{e} \mu^{-} \bar{\nu}_{\mu} b \bar{b} j+X$ process at the LHC run II with $\sqrt{s}=13 \mathrm{TeV}$.

\subsection{Theoretical uncertainties for differential cross sections}

At this point we would like to fully assess the uncertainties inherent in our NLO differential predictions. An extensive discussion of the scale uncertainties has already been presented in the previous section, based on a fixed PDF choice (CT14). In this section, we complete our analysis by studying comparatively the impact of PDF and scale variations on the overall theoretical uncertainty. Judging by the dependence of the total cross section, the PDF uncertainties should be below or of the same order as the theoretical uncertainties predicted by the scale variation. Nevertheless, we would like to examine this carefully for all differential cross sections that we have presented in the previous section. 

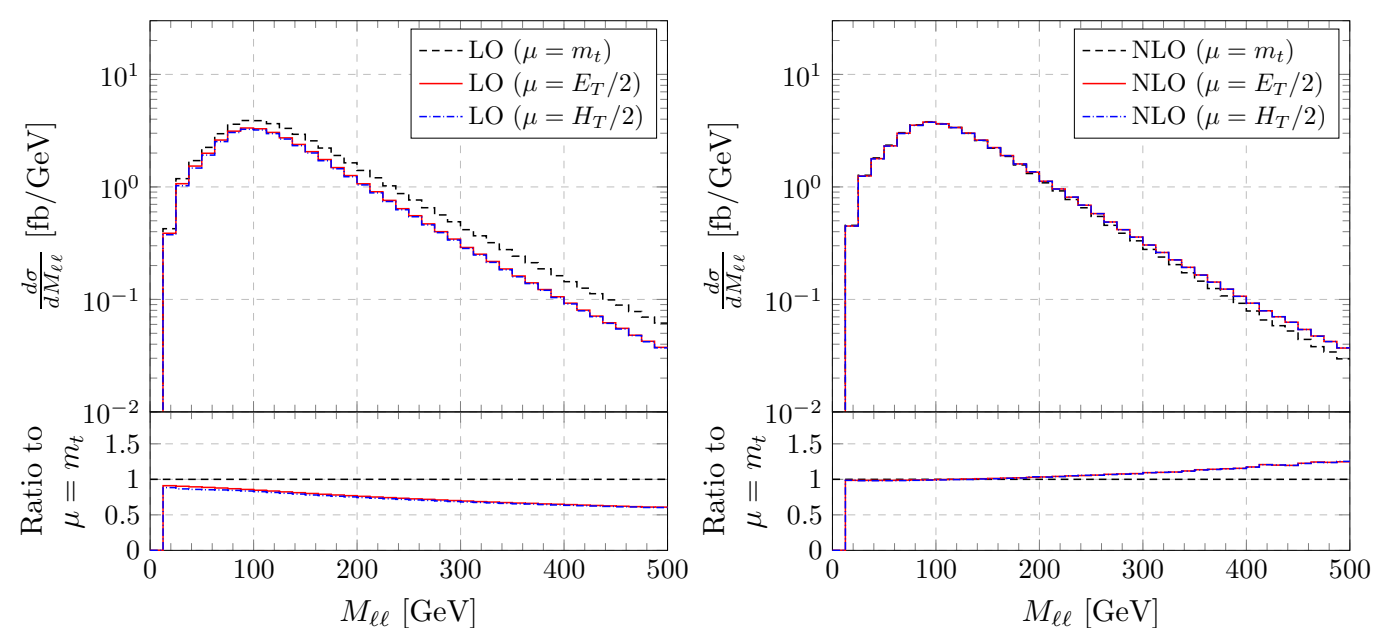

Figure 26. Differential cross section distributions as a function of the invariant mass of two charged leptons at LO (left panel) and at NLO (right panel) for the $p p \rightarrow e^{+} \nu_{e} \mu^{-} \bar{\nu}_{\mu} b \bar{b} j+X$ process at the LHC run II with $\sqrt{s}=13 \mathrm{TeV}$.

To this end we plot afresh NLO differential cross sections for our best (dynamical) scale choice, $\mu_{0}=H_{T} / 2$, for three different PDF sets, CT14, MMHT14 and NNPDF3.0. We shall start with the averaged distribution of the transverse momentum of the top quark, bottom-jet and charged lepton that are shown in figure 33. Also given there is the transverse momentum distribution of the hardest light-jet. Each figure comprises three parts; the upper panel shows the NLO prediction for three different PDF sets at the central scale value, $\mu_{R}=\mu_{F}=\mu_{0}=H_{T} / 2$, the middle panel displays the NLO scale-dependence band normalised to the central CT14 NLO prediction, whereas the lower panel gives the PDF uncertainties obtained for each PDF set separately, again normalised to the central NLO prediction as obtained with the CT14 PDF set. For each observable plotted in figure 33 we obtain symmetrised scale uncertainties below $10 \%$ (with respect to the central value). To be more specific we have estimated $8 \%$ scale uncertainties for $p_{T, t}$ at the beginning of the spectrum, which decreased down to $4 \%$ in the tails. In these high $p_{T}$ regions, however, PDF uncertainties are of a comparable size, i.e. they are of the order of $6 \%$ for CT14 and $3 \%$ for MMHT14 and NNPDF3.0 (again with respect to the corresponding central values). For the transverse momentum distribution of the bottom-jet we have a different behaviour, namely scale uncertainties have increased in the tails and reached almost $10 \%$ while PDF uncertainties stayed below 6\% (3\%) for CT14 (for MMHT14 and NNPDF3.0). For the transverse momentum distribution of the charged lepton we find that the scale variations are of the order of the error of the CT14 PDFs, i.e. below 6\%. The other two PDF sets show a smaller uncertainty, of the order of $3 \%$. For the hardest light jet in the whole plotted range CT14 PDF uncertainties are below scale uncertainties. The former are estimated to be below $5 \%$ the latter below 9\%. For MMHT14 and NNPDF3.0 PDF sets we have respectively $3 \%$ and $2 \%$ PDF uncertainties.

In figure 34 we present the invariant mass of the $t \bar{t}$ system, $M_{t \bar{t}}$, of two charged leptons, $M_{\ell \ell}$, and of bottom-jet and positron, $M_{b e^{+}}$, together with the mass of the reconstructed top quark, $M_{t}$. We start with the invariant mass of the $t \bar{t}$ pair. In the vicinity of the $t \bar{t}$ 

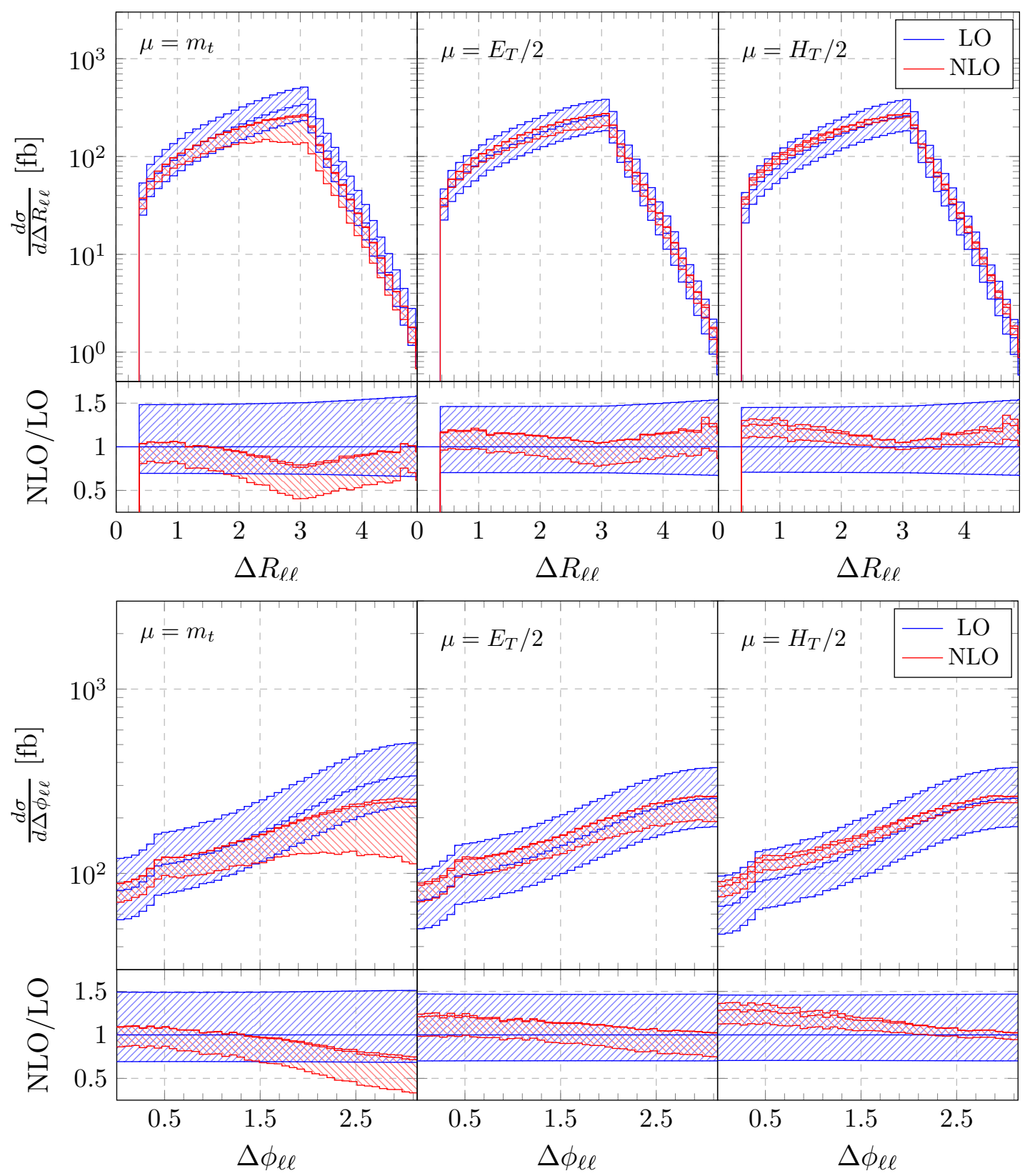

Figure 27. Differential cross section distribution as a function of $\Delta R_{\ell \ell}$ and $\Delta \phi_{\ell \ell}$ for the $p p \rightarrow e^{+} \nu_{e} \mu^{-} \bar{\nu}_{\mu} b \bar{b} j+X$ process at the LHC run II with $\sqrt{s}=13 \mathrm{TeV}$.

threshold sizeable, of the order of $30 \%$, scale uncertainties are attained. However, starting from about $400 \mathrm{GeV}$ almost constant $5 \%$ uncertainties are noticed until the end of the plotted spectrum, i.e. up to $1.5 \mathrm{TeV}$. As for the PDF uncertainties we observe $7 \%$ effects in the tail of this distribution in case of CT14 and $4 \%$ for MMHT14 and NNPDF3.0. For the invariant mass of the positron-muon system, $M_{\ell \ell}$, we observe $7 \%$ scale uncertainties for small values, decreasing down to $5 \%$ after $100 \mathrm{GeV}$. Thus, around $500 \mathrm{GeV}$ they are comparable to CT14 PDF uncertainties that are of the order of $6 \%$. Also here this effect is 

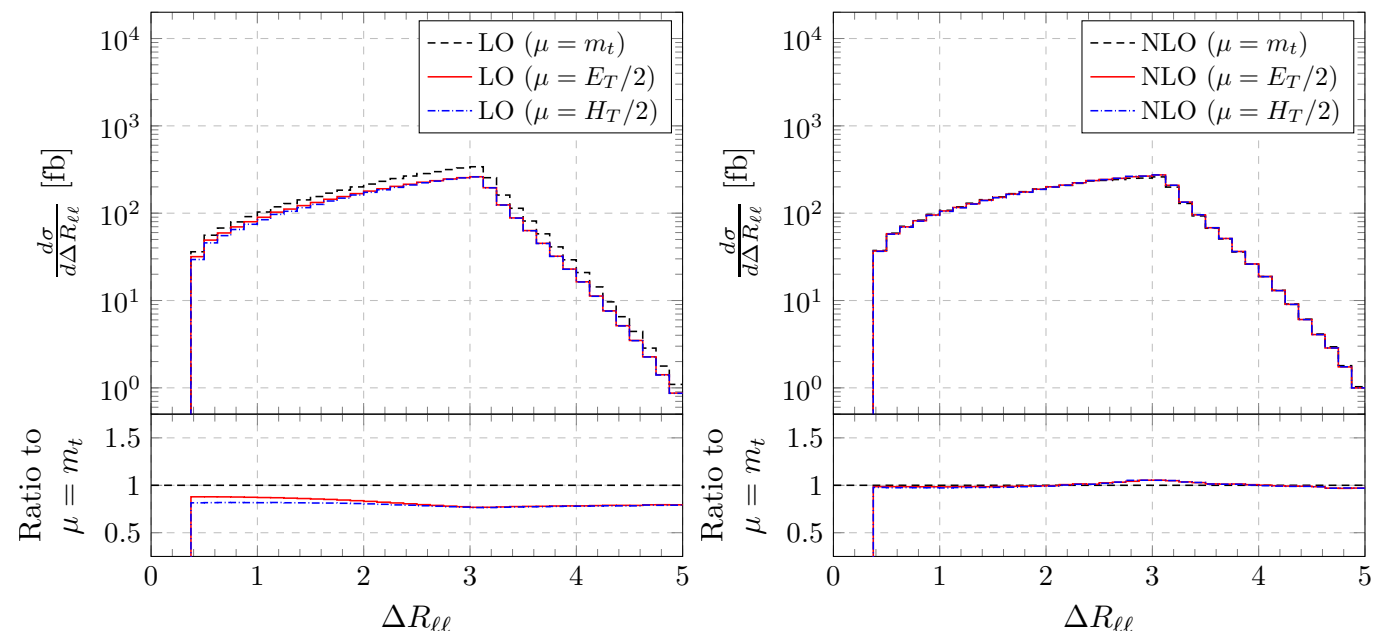

Figure 28. Differential cross section distributions as a function of $\Delta R_{\ell \ell}$ at LO (left panel) and at NLO (right panel) for the $p p \rightarrow e^{+} \nu_{e} \mu^{-} \bar{\nu}_{\mu} b \bar{b} j+X$ process at the LHC run II with $\sqrt{s}=13 \mathrm{TeV}$.
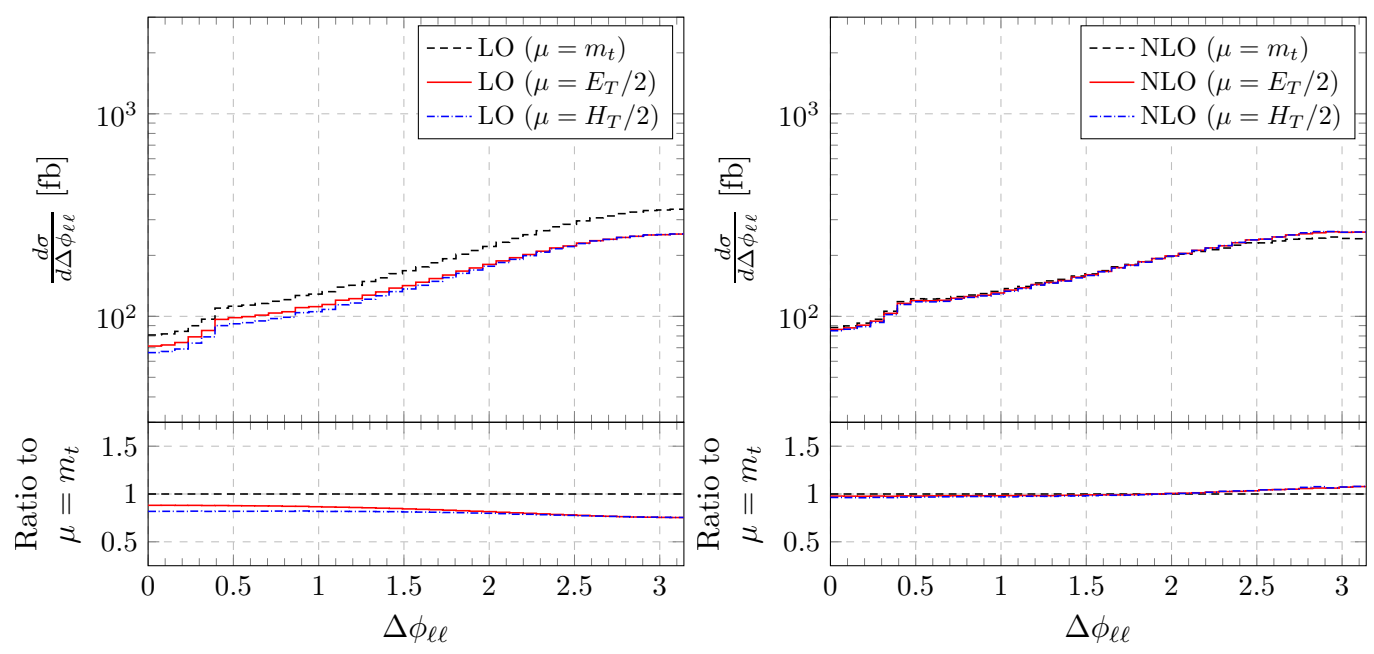

Figure 29. Differential cross section distributions as a function of $\Delta \phi_{\ell \ell}$ at LO (left panel) and at NLO (right panel) for the $p p \rightarrow e^{+} \nu_{e} \mu^{-} \bar{\nu}_{\mu} b \bar{b} j+X$ process at the LHC run II with $\sqrt{s}=13 \mathrm{TeV}$.

smaller for MMHT14 and NNPDF3.0 (4\% and 3\%). The invariant mass of the bottom-jet and positron, $M_{b e^{+}}$, has clearly two distinct ranges when it comes to scale uncertainties. Up to the kinematical endpoint they are of the order of $6 \%$, on the other hand, above this point they reach $30 \%$. PDF uncertainties, as expected, do not affect $M_{b e^{+}}^{\max }$ and are of the order of $2 \%-4 \%$ in the whole range independently of the PDF set. The mass of the reconstructed top quark, that is presented close to the resonance, has a more complex pattern. Even for such a small range, i.e. $167-177 \mathrm{GeV}$, we can distinguish three different regions. Up to $170 \mathrm{GeV}$ scale uncertainties are within the $20 \%-30 \%$ range, they are decreased down to $10 \%-15 \%$ for $M_{t} \in(170-174) \mathrm{GeV}$ and are further reduced below $10 \%$ for $M_{t}>174 \mathrm{GeV}$. One more time, PDF uncertainties remain the same in the whole plotted range and are of the order of $2 \%-3 \%$, well below scale uncertainties, independently of the PDF set used. 

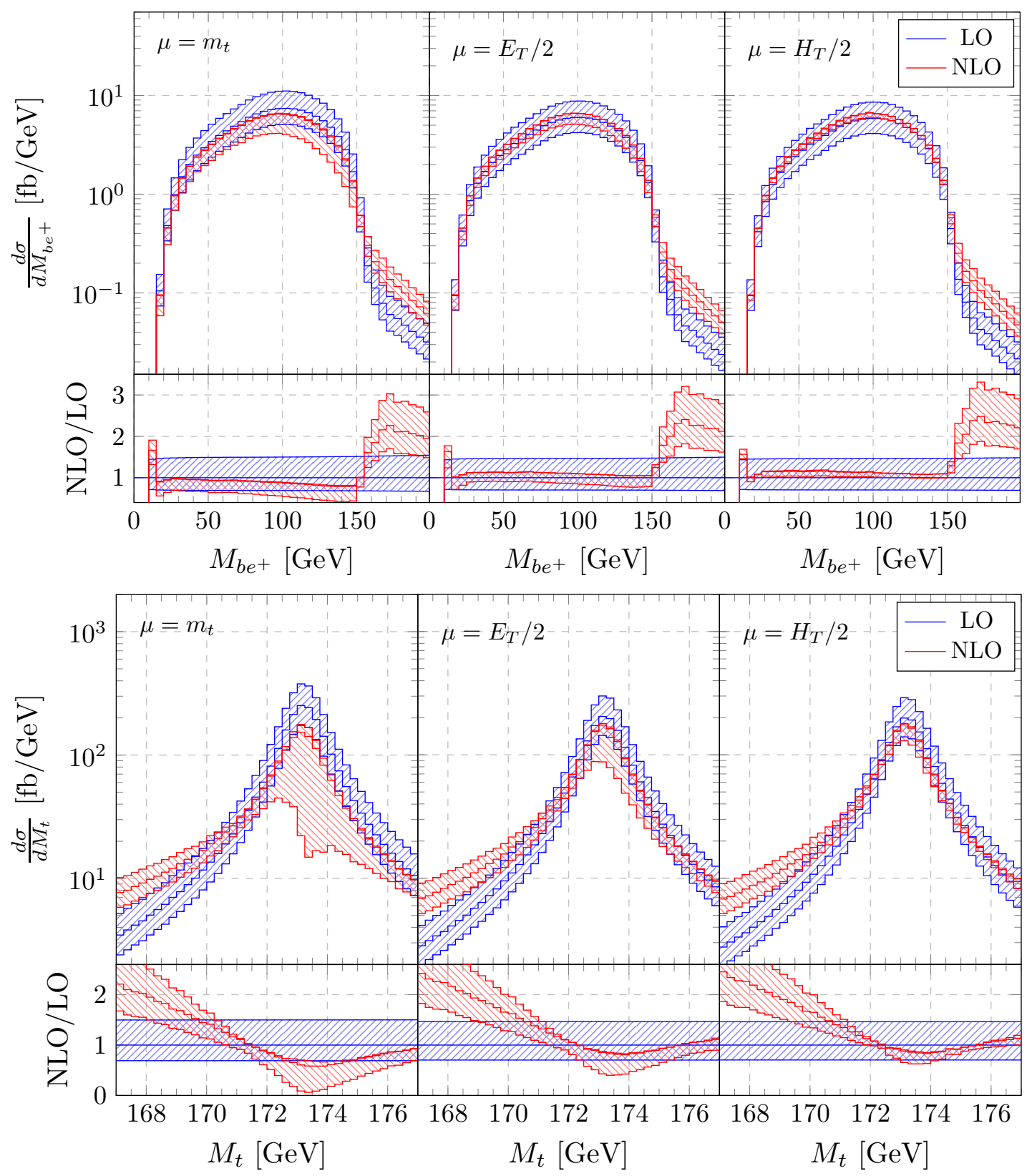

Figure 30. Differential cross section distribution as a function of the invariant mass of the positron and bottom-jet and the averaged differential cross section distribution as a function of invariant mass of the top quark for the $p p \rightarrow e^{+} \nu_{e} \mu^{-} \bar{\nu}_{\mu} b \bar{b} j+X$ process at the LHC run II with $\sqrt{s}=13 \mathrm{TeV}$.

In figure 35 we show dimensionless observables, namely rapidity distributions for the top quark, bottom jet, charged lepton and the hardest light-jet. In the central rapidity regions of $y_{t}$ scale uncertainties are of the order of $5 \%$, whereas they reach $10 \%$ at the peripheral parts of the distribution. The CT14 (MMHT14, NNPDF3.0) PDF uncertainties are at the level of $3 \%(2 \%)$ and $7 \%(4 \%$ and $5 \%)$ in these two distinct regions. In case of the rapidity distribution of the bottom-jet scale uncertainties are below $8 \%$ and the PDF uncertainties are in the range $3 \%-5 \%$. A similar pattern could be recognised for $y_{\ell}$ and $y_{j_{1}}$. 

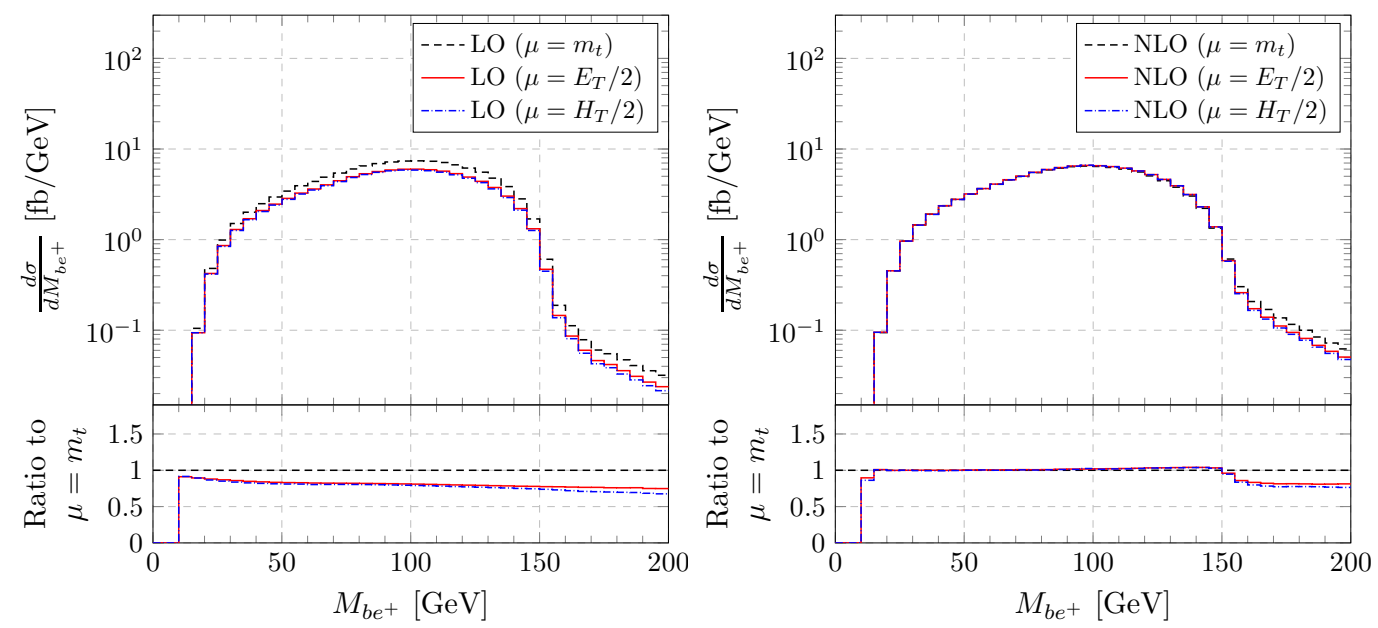

Figure 31. Differential cross section distributions as a function of the invariant mass of the bottomjet and positron at LO (left panel) and at NLO (right panel) for the $p p \rightarrow e^{+} \nu_{e} \mu^{-} \bar{\nu}_{\mu} b \bar{b} j+X$ process at the LHC run II with $\sqrt{s}=13 \mathrm{TeV}$.
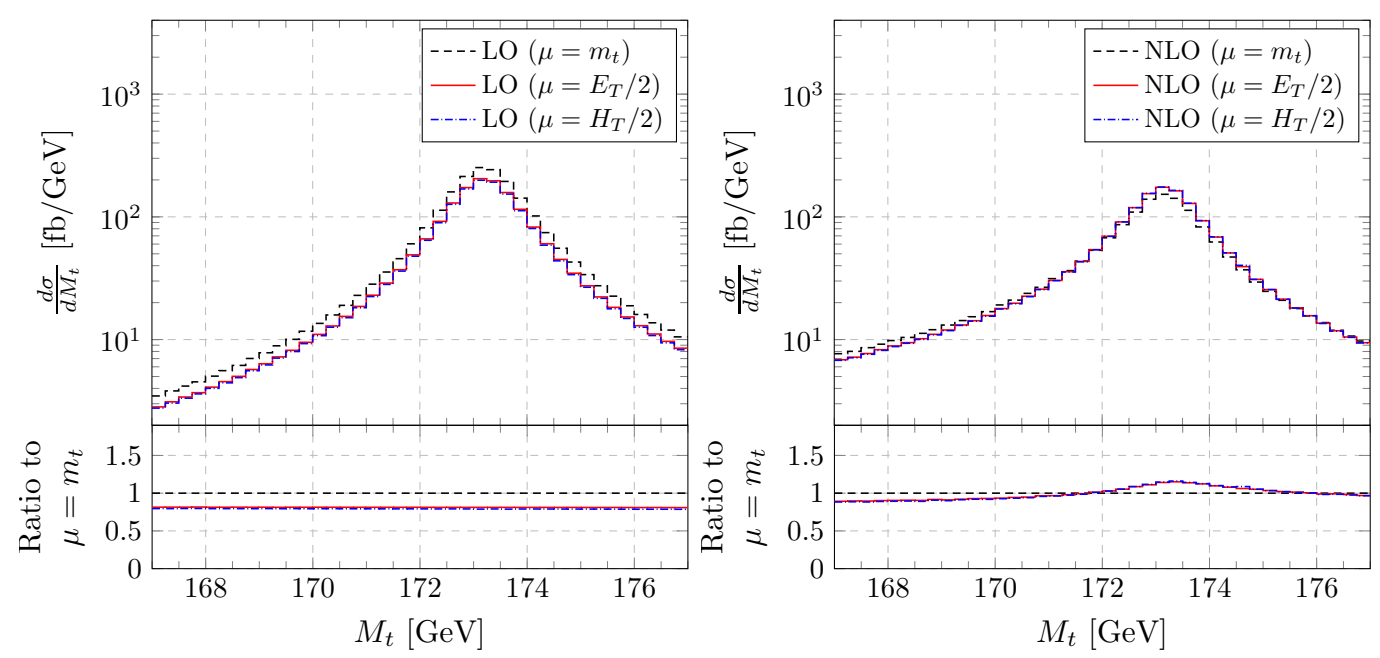

Figure 32. Averaged differential cross section distributions as a function of the invariant mass of the top quark at LO (left panel) and at NLO (right panel) for the $p p \rightarrow e^{+} \nu_{e} \mu^{-} \bar{\nu}_{\mu} b \bar{b} j+X$ process at the LHC run II with $\sqrt{s}=13 \mathrm{TeV}$.

Finally, in figure 36 we plot the total transverse momentum of the $t \bar{t} j$ system, the missing transverse momentum, $\Delta R_{\ell \ell}$ and $\Delta \phi_{\ell \ell}$. For the $H_{T}$ distribution, scale uncertainties are below $10 \%$. In the high $p_{T}$ region they are comparable to the CT14 PDF uncertainties that are of the order of $7 \%$. For the other PDF sets we obtained PDF uncertainties below $4 \%$. Qualitatively comparable conclusions have been reached for the $p_{T}$ distribution. Also for $\Delta R_{\ell \ell}$ and $\Delta \phi_{\ell \ell}$ distributions, we have estimated scale uncertainties below $10 \%$. The PDF uncertainties for $\Delta R_{\ell \ell}$ have been found to be below $6 \%, 3.5 \%$ and $3 \%$ for the CT14, MMHT14 and NNPDF3.0 PDF sets respectively. In the case of $\Delta \phi_{\ell \ell}$ they are slightly smaller, i.e. $4 \%$ for CT14 and 2\% for the MMHT14 and NNPDF3.0 PDF sets. 

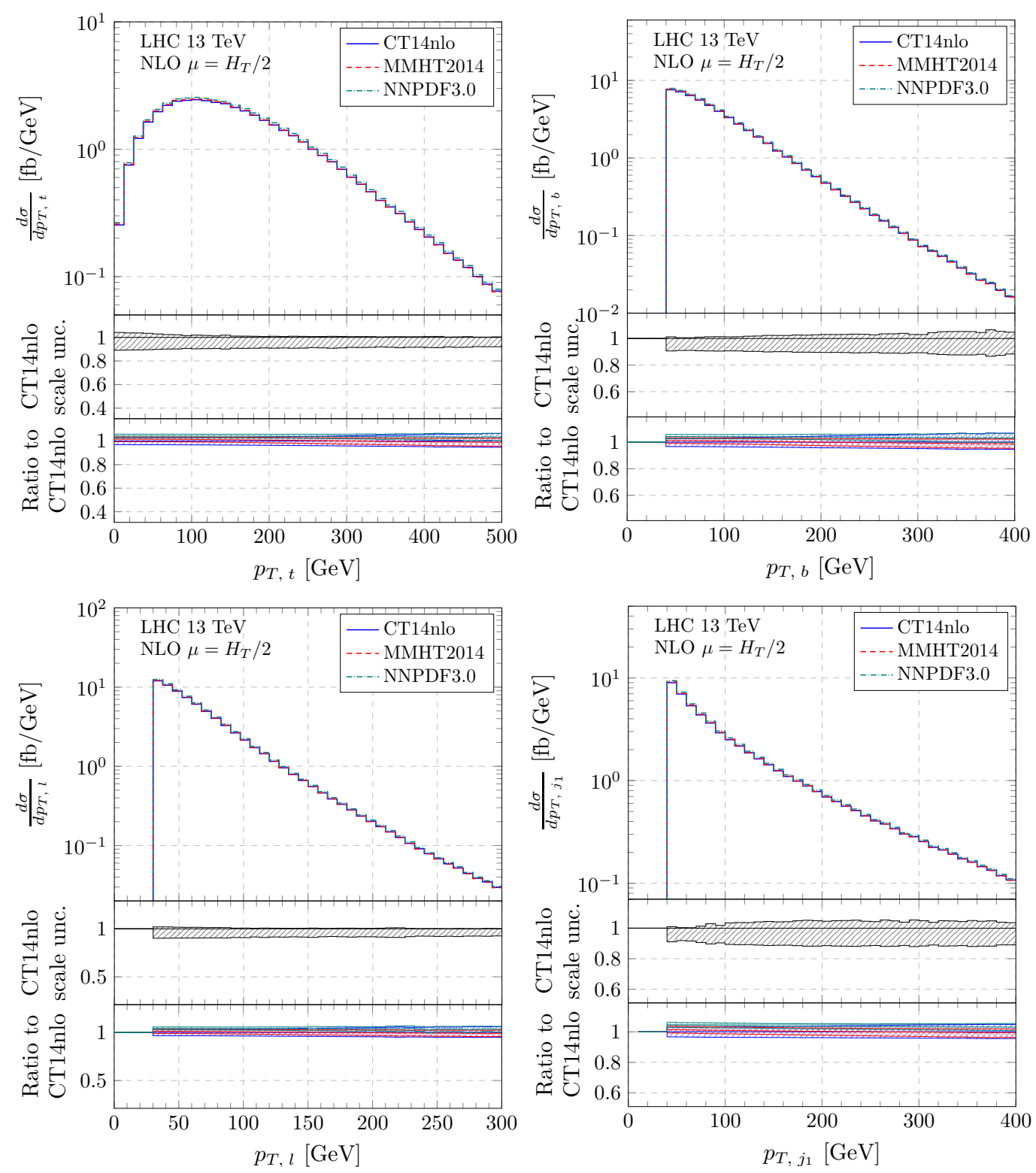

Figure 33. Averaged NLO differential cross section distributions as a function of the transverse momentum of the top quark, bottom-jet and charged lepton. Also given is the NLO differential cross section as a function of the transverse momentum of the hardest light jet. Results are shown for the $p p \rightarrow e^{+} \nu_{e} \mu^{-} \bar{\nu}_{\mu} b \bar{b} j+X$ process at the LHC run II with $\sqrt{s}=13 \mathrm{TeV}$ for three different PDF sets. Lower panels display scale and PDF uncertainties of the NLO cross section normalised to the central NLO prediction with CT14.

\section{Conclusions}

In this paper, we have presented a comprehensive NLO study of the off-shell production of $t \bar{t}+$ jet with leptonic decays of the top quarks. All results have been obtained by use of the package HELAC-NLO. We have shown predictions for total cross sections and distributions for a variety of observables of phenomenological interest for the LHC Run 

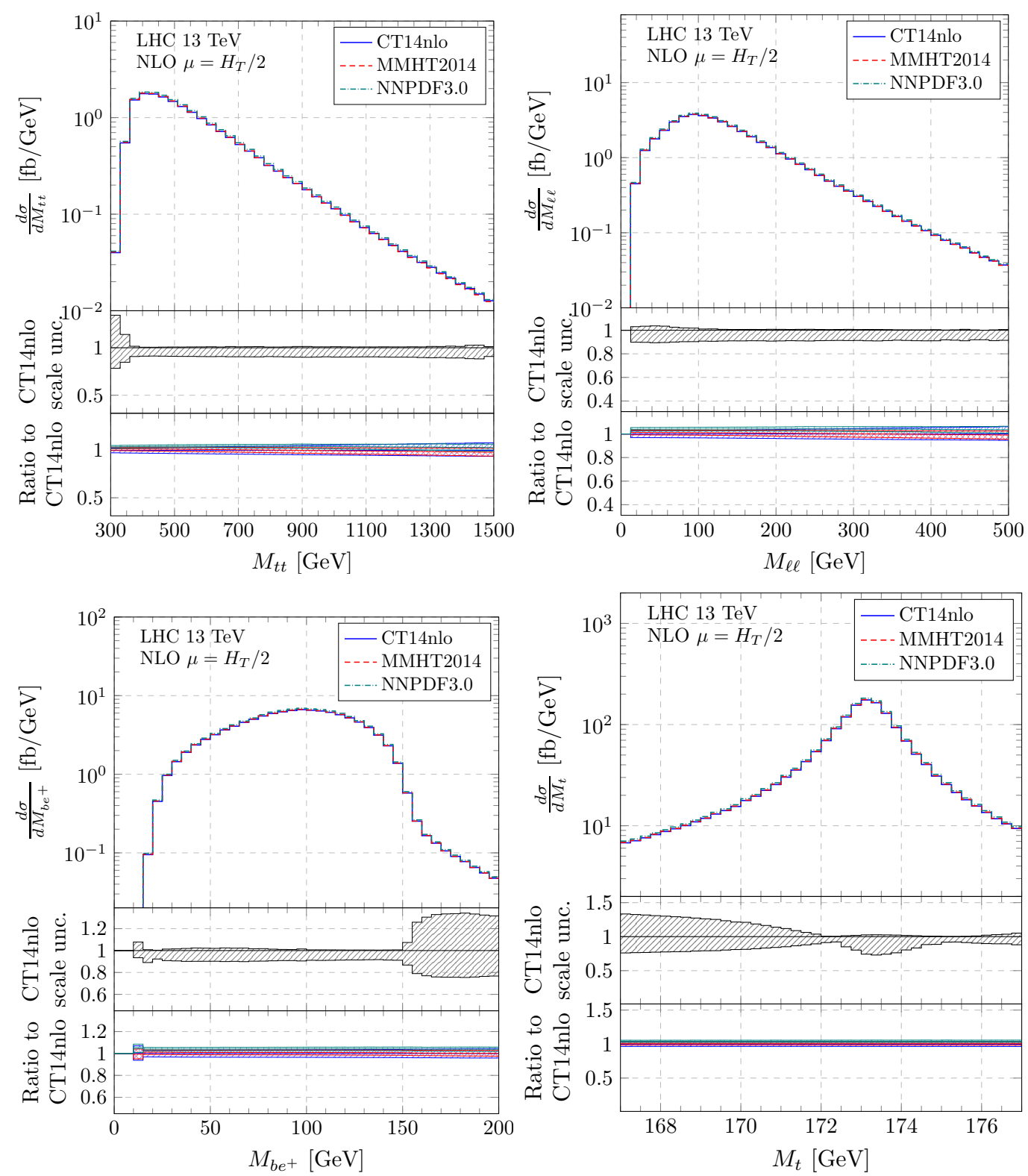

Figure 34. NLO differential cross section distributions as a function of the invariant mass of the $t \bar{t}$ system, charged leptons and bottom jet and positron. Also given is the averaged NLO differential cross section distribution as a function of the invariant mass of the top quark. Results are shown for the $p p \rightarrow e^{+} \nu_{e} \mu^{-} \bar{\nu}_{\mu} b \bar{b} j+X$ process at the LHC run II with $\sqrt{s}=13$.

II energy of $13 \mathrm{TeV}$. Also, we have carefully assessed the theoretical uncertainties of our predictions stemming from scale dependence and from different PDF parametrizations. For our best scale choice, $\mu_{R}=\mu_{F}=H_{T} / 2$, the QCD corrections to the total cross section are positive and vary from rather small to moderate. To be more specific, we have obtained corrections of the order $15 \%$ for the CT14 PDF set, $6 \%$ for MMHT14 and $24 \%$ for NNPDF3.0. As to the theoretical uncertainties, taking them conservatively from the upper and lower results, we have observed a reduction from $50 \%$ at LO down to $10 \%$ at the 

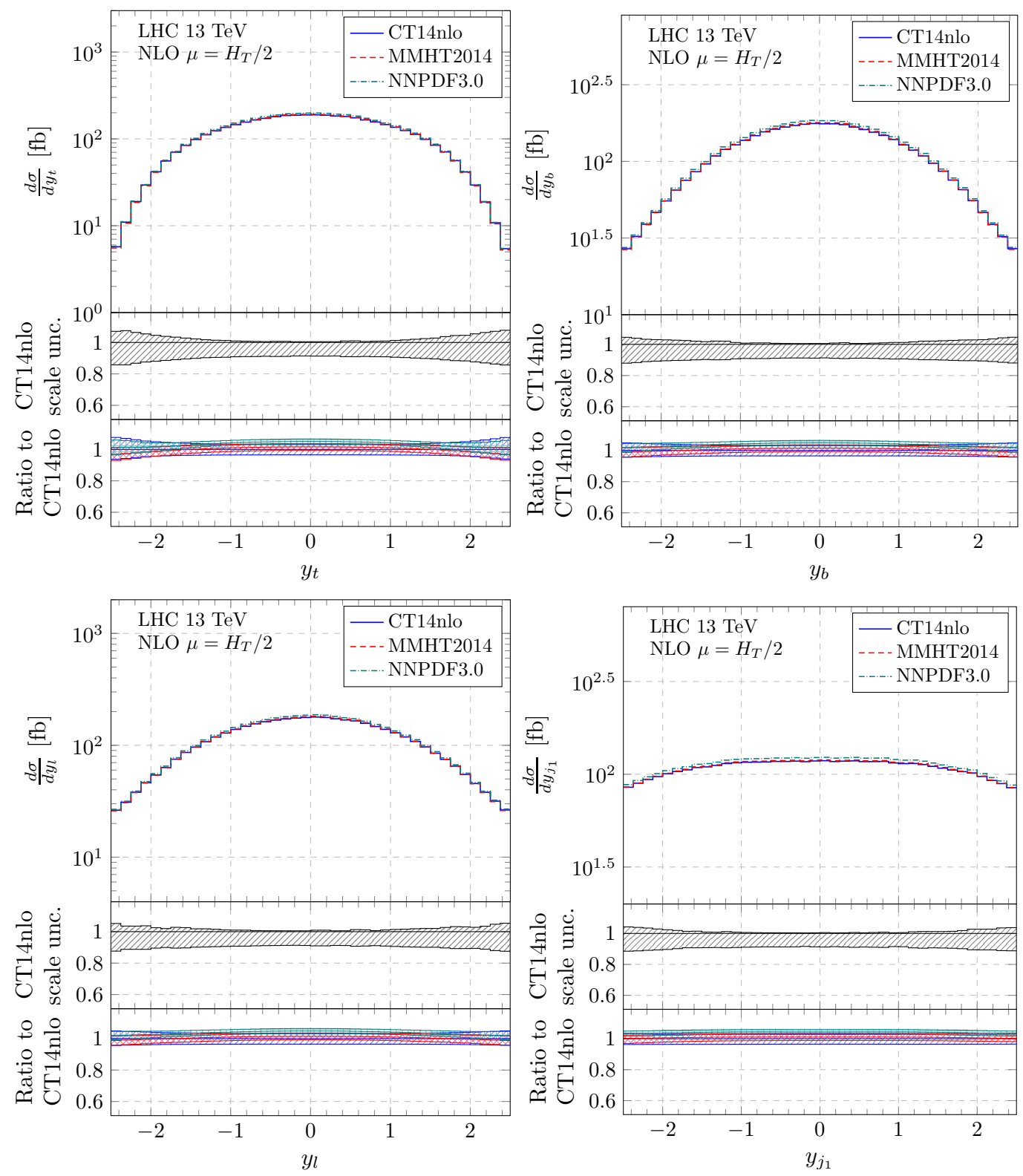

Figure 35. Averaged NLO differential cross section distributions as a function of the rapidity of the top quark, bottom-jet and charged lepton. Also given is the NLO differential cross section as a function of the rapidity of the hardest light jet. Results are shown for the $p p \rightarrow e^{+} \nu_{e} \mu^{-} \bar{\nu}_{\mu} b \bar{b} j+X$ process at the LHC run II with $\sqrt{s}=13 \mathrm{TeV}$.

NLO. Using symmetrization, the scale uncertainties become $40 \%$ at LO and $6 \%$ at NLO. The PDF uncertainties have been assessed to be rather small at the inclusive level, within the range of $2 \%-3 \%$. Moreover, results have been found to be quite stable for cuts on the $p_{T}$ of the hard jet ranging from $40 \mathrm{GeV}$ to $120 \mathrm{GeV}$.

We have considered several differential distributions which are relevant for the ongoing analyses at the LHC. Two different dynamical scales have been considered for our analysis, $\mu_{R}=\mu_{F}=E_{T} / 2$ and $\mu_{R}=\mu_{F}=H_{T} / 2$, which proved both effective in stabilizing the 

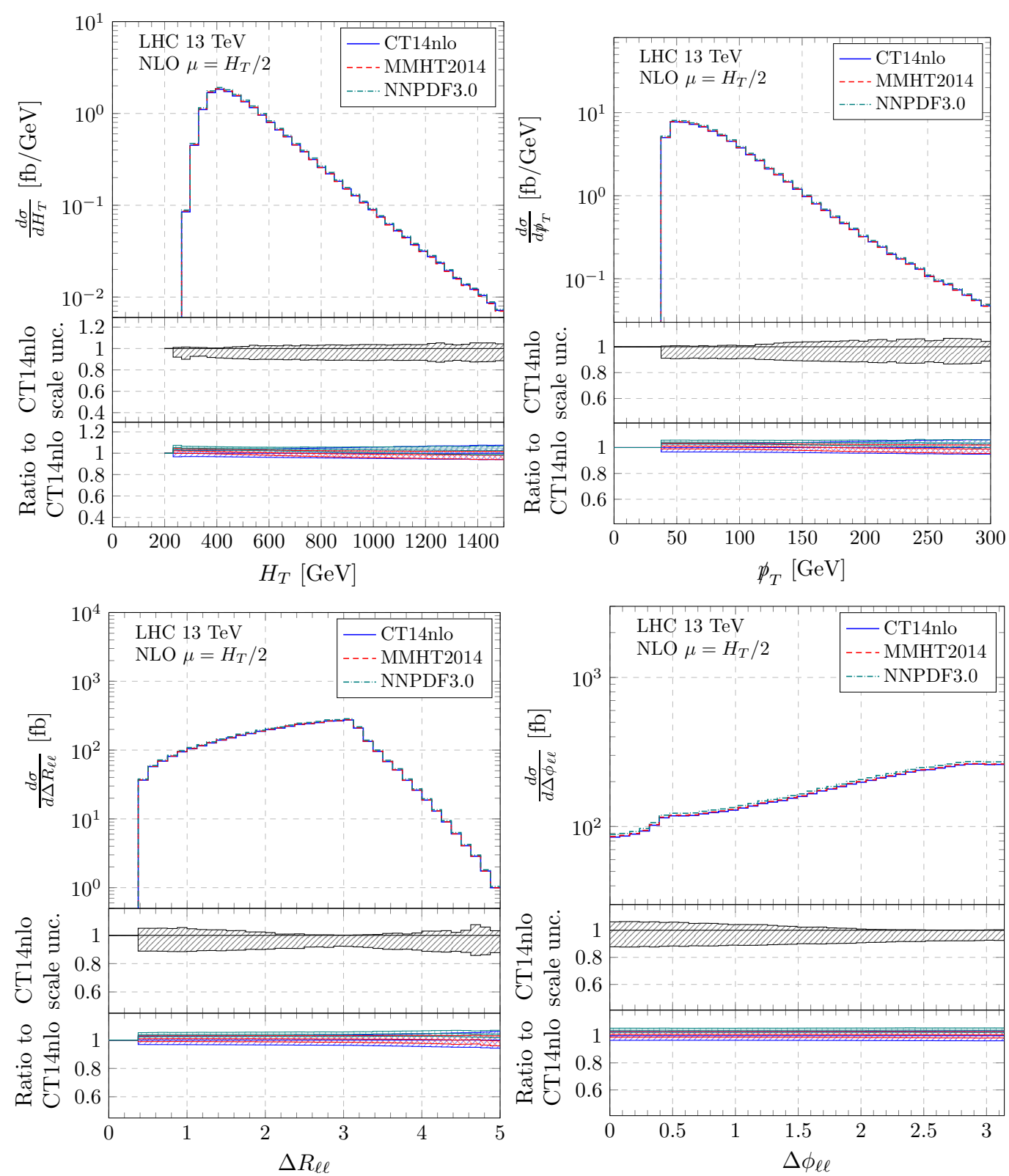

Figure 36. NLO differential cross section distributions as a function of the total transverse momentum of the system, missing transverse momentum, $\Delta R_{\ell \ell}$ and $\Delta \phi_{\ell \ell}$. Results are shown for the $p p \rightarrow e^{+} \nu_{e} \mu^{-} \bar{\nu}_{\mu} b \bar{b} j+X$ process at the LHC run II with $\sqrt{s}=13 \mathrm{TeV}$.

perturbative convergence in phase space regions far away from the $2 m_{t}$ threshold. Of the two scales, $H_{T} / 2$ is the one which provides the smallest theoretical uncertainties as estimated by the scale variation. The size of the QCD corrections varies considerably from observable to observable. For the majority of cases we have found that corrections are below 10\%-20\%, yet they can exceed $100 \%$ for specific observables independently of the scale choice. At the differential level, PDF uncertainties are found to be of comparable size, i.e. below $10 \%$, thus they cannot simply be neglected. This fact is particularly evident 
using the CT14 PDF set, while the uncertainties related to the MMHT14 and NNPDF3.0 sets are within the scale dependence ones.

In the next step, we plan to use our predictions to study broad phenomenological aspects of top quark physics at the LHC. Our priority is to assist precise measurements of the top quark mass at the LHC, where the impact of the off-shell effects has to be carefully examined in order to assess realistically the theoretical uncertainties. To this end, a systematic comparison with predictions based on the narrow-width approximation is required. We also plan to quantify the impact of b-quark mass effects at NLO by means of comparisons between the so-called Five-Flavour and Four-Flavour schemes.

\section{Acknowledgments}

The work of H. B. Hartanto and M. Worek was supported by the German Research Foundation (DFG). M. Kraus acknowledges support by the German Federal Ministry of Education and Research (BMBF). Authors would like to thank Adam Kardos for help with the QGRAF program.

Open Access. This article is distributed under the terms of the Creative Commons Attribution License (CC-BY 4.0), which permits any use, distribution and reproduction in any medium, provided the original author(s) and source are credited.

\section{References}

[1] D.L. Rainwater, R. Szalapski and D. Zeppenfeld, Probing color singlet exchange in $Z+$ two jet events at the CERN LHC, Phys. Rev. D 54 (1996) 6680 [hep-ph/9605444] [INSPIRE].

[2] V. Del Duca, A. Frizzo and F. Maltoni, Higgs boson production in association with three jets, JHEP 05 (2004) 064 [hep-ph/0404013] [INSPIRE].

[3] T. Figy, V. Hankele and D. Zeppenfeld, Next-to-leading order QCD corrections to Higgs plus three jet production in vector-boson fusion, JHEP 02 (2008) 076 [arXiv:0710.5621] [INSPIRE].

[4] M.L. Mangano, Standard Model backgrounds to supersymmetry searches, Eur. Phys. J. C 59 (2009) 373 [arXiv:0809.1567] [INSPIRE].

[5] C. Englert, B. Jager, M. Worek and D. Zeppenfeld, Observing Strongly Interacting Vector Boson Systems at the CERN Large Hadron Collider, Phys. Rev. D 80 (2009) 035027 [arXiv:0810.4861] [INSPIRE].

[6] C. Englert, B. Jager and D. Zeppenfeld, QCD Corrections to Vector-Boson Fusion Processes in Warped Higgsless Models, JHEP 03 (2009) 060 [arXiv:0812.2564] [INSPIRE].

[7] F. Campanario, M. Kubocz and D. Zeppenfeld, Gluon-fusion contributions to $\Phi+2$ Jet production, Phys. Rev. D 84 (2011) 095025 [arXiv: 1011.3819] [InSPIRE].

[8] D.L. Rainwater and D. Zeppenfeld, Observing $H \rightarrow W^{*} W^{*} \rightarrow e^{ \pm} \mu \mp p_{T}$ in weak boson fusion with dual forward jet tagging at the CERN LHC, Phys. Rev. D 60 (1999) 113004 [Erratum ibid. D 61 (2000) 099901] [hep-ph/9906218] [INSPIRE].

[9] N. Kauer, T. Plehn, D.L. Rainwater and D. Zeppenfeld, $H \rightarrow W^{+} W^{-}$as the discovery mode for a light Higgs boson, Phys. Lett. B 503 (2001) 113 [hep-ph/0012351] [INSPIRE]. 
[10] M.I. Gresham, I.-W. Kim and K.M. Zurek, Searching for Top Flavor Violating Resonances, Phys. Rev. D 84 (2011) 034025 [arXiv:1102.0018] [inSPIRE].

[11] ATLAS collaboration, Search for resonant top plus jet production in $t \bar{t}+$ jets events with the ATLAS detector in pp collisions at $\sqrt{s}=7$ TeV, Phys. Rev. D 86 (2012) 091103 [arXiv: 1209.6593] [INSPIRE].

[12] CMS collaboration, Search for charge-asymmetric production of $W^{\prime}$ bosons in $t \bar{t}+$ jet events from pp collisions at $\sqrt{s}=7$ TeV, Phys. Lett. B 717 (2012) 351 [arXiv:1206.3921] [INSPIRE].

[13] W. Buchmüller and D. Wyler, Effective Lagrangian Analysis of New Interactions and Flavor Conservation, Nucl. Phys. B 268 (1986) 621 [INSPIRE].

[14] J.A. Aguilar-Saavedra, A Minimal set of top anomalous couplings, Nucl. Phys. B 812 (2009) 181 [arXiv:0811.3842] [INSPIRE].

[15] J.A. Aguilar-Saavedra, B. Fuks and M.L. Mangano, Pinning down top dipole moments with ultra-boosted tops, Phys. Rev. D 91 (2015) 094021 [arXiv:1412.6654] [INSPIRE].

[16] CMS collaboration, Search for Anomalous Top Chromomagnetic Dipole Moments from angular distributions in $t \bar{t}$ Dileptonic events at $\sqrt{s}=7 \mathrm{TeV}$ with the CMS detector., CMS-PAS-TOP-14-005.

[17] CMS collaboration, Measurements of $t$ t-bar spin correlations and top quark polarization using dilepton final states in pp collisions at $\sqrt{s}=8$ TeV, Phys. Rev. D 93 (2016) 052007 [arXiv: 1601.01107] [INSPIRE].

[18] S. Alioli et al., A new observable to measure the top-quark mass at hadron colliders, Eur. Phys. J. C 73 (2013) 2438 [arXiv: 1303.6415] [inSPIRE].

[19] ATLAS collaboration, Determination of the top-quark pole mass using $t \bar{t}+1$-jet events collected with the ATLAS experiment in 7 TeV pp collisions, JHEP 10 (2015) 121 [arXiv: 1507.01769] [INSPIRE].

[20] CMS collaboration, Determination of the normalised invariant mass distribution of $\mathrm{t} \overline{\mathrm{t}}+$ jet and extraction of the top quark mass, CMS-PAS-TOP-13-006.

[21] ATLAS collaboration, Measurement of t $\bar{t}$ production with a veto on additional central jet activity in pp collisions at $\sqrt{s}=7 \mathrm{TeV}$ using the ATLAS detector, Eur. Phys. J. C 72 (2012) 2043 [arXiv: 1203.5015] [INSPIRE].

[22] CMS collaboration, Measurement of jet multiplicity distributions in $\mathrm{t} \overline{\mathrm{t}}$ production in pp collisions at $\sqrt{s}=7 \mathrm{TeV}$, Eur. Phys. J. C 74 (2015) 3014 [Erratum ibid. C 75 (2015) 216] [arXiv: 1404.3171] [INSPIRE].

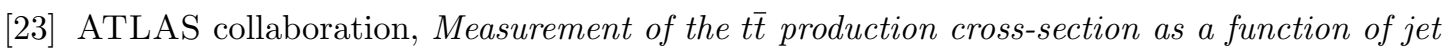
multiplicity and jet transverse momentum in 7 TeV proton-proton collisions with the ATLAS detector, JHEP 01 (2015) 020 [arXiv:1407.0891] [INSPIRE].

[24] ATLAS collaboration, Measurements of fiducial cross-sections for ti production with one or two additional b-jets in pp collisions at $\sqrt{s}=8 \mathrm{TeV}$ using the ATLAS detector, Eur. Phys. J. C 76 (2016) 11 [arXiv:1508.06868] [INSPIRE].

[25] CMS collaboration, Measurement of $\mathrm{t} \overline{\mathrm{t}}$ production with additional jet activity, including $\mathrm{b}$ quark jets, in the dilepton decay channel using pp collisions at $\sqrt{s}=8 \mathrm{TeV}$, Eur. Phys. J. C 76 (2016) 379 [arXiv:1510.03072] [INSPIRE]. 
[26] ATLAS collaboration, Measurement of jets produced in top quark events using the di-lepton final state with 2 b-tagged jets in pp collisions at $\sqrt{s}=13 \mathrm{TeV}$ with the ATLAS detector, ATLAS-CONF-2015-065.

[27] ATLAS collaboration, Measurement of jet activity in top quark events using the e $\mu$ final state with two b-tagged jets in pp collisions at $\sqrt{s}=8 \mathrm{TeV}$ with the ATLAS detector, JHEP 09 (2016) 074 [arXiv: 1606.09490] [INSPIRE].

[28] CMS collaboration, Measurement of the differential production cross section for top-quark pairs as a function of jet multiplicity in the lepton+jets final state at $\sqrt{s}=8 \mathrm{TeV}$ with the CMS detector, CMS-PAS-TOP-15-006.

[29] S. Dittmaier, P. Uwer and S. Weinzierl, NLO QCD corrections to $t \bar{t}+$ jet production at hadron colliders, Phys. Rev. Lett. 98 (2007) 262002 [hep-ph/0703120] [INSPIRE].

[30] S. Dittmaier, P. Uwer and S. Weinzierl, Hadronic top-quark pair production in association with a hard jet at next-to-leading order QCD: Phenomenological studies for the Tevatron and the LHC, Eur. Phys. J. C 59 (2009) 625 [arXiv:0810.0452] [InSPIRE].

[31] K. Melnikov and M. Schulze, NLO QCD corrections to top quark pair production in association with one hard jet at hadron colliders, Nucl. Phys. B 840 (2010) 129 [arXiv: 1004.3284] [INSPIRE].

[32] K. Melnikov, A. Scharf and M. Schulze, Top quark pair production in association with a jet: QCD corrections and jet radiation in top quark decays, Phys. Rev. D 85 (2012) 054002 [arXiv:1111.4991] [INSPIRE].

[33] A. Kardos, C. Papadopoulos and Z. Trócsányi, Top quark pair production in association with a jet with NLO parton showering, Phys. Lett. B 705 (2011) 76 [arXiv:1101.2672] [INSPIRE].

[34] S. Alioli, S.-O. Moch and P. Uwer, Hadronic top-quark pair-production with one jet and parton showering, JHEP 01 (2012) 137 [arXiv:1110.5251] [INSPIRE].

[35] M. Czakon, H.B. Hartanto, M. Kraus and M. Worek, Matching the Nagy-Soper parton shower at next-to-leading order, JHEP 06 (2015) 033 [arXiv:1502.00925] [INSPIRE].

[36] P. Nason, A new method for combining NLO QCD with shower Monte Carlo algorithms, JHEP 11 (2004) 040 [hep-ph/0409146] [INSPIRE].

[37] S. Frixione, P. Nason and C. Oleari, Matching NLO QCD computations with Parton Shower simulations: the POWHEG method, JHEP 11 (2007) 070 [arXiv:0709.2092] [INSPIRE].

[38] S. Alioli, P. Nason, C. Oleari and E. Re, A general framework for implementing NLO calculations in shower Monte Carlo programs: the POWHEG BOX, JHEP 06 (2010) 043 [arXiv: 1002.2581] [INSPIRE].

[39] S. Frixione and B.R. Webber, Matching NLO QCD computations and parton shower simulations, JHEP 06 (2002) 029 [hep-ph/0204244] [INSPIRE].

[40] G. Bevilacqua, H.B. Hartanto, M. Kraus and M. Worek, Top Quark Pair Production in Association with a Jet with Next-to-Leading-Order QCD Off-Shell Effects at the Large Hadron Collider, Phys. Rev. Lett. 116 (2016) 052003 [arXiv: 1509. 09242] [inSPIRE].

[41] A. Denner, S. Dittmaier, S. Kallweit and S. Pozzorini, NLO QCD corrections to WWbb production at hadron colliders, Phys. Rev. Lett. 106 (2011) 052001 [arXiv:1012.3975] [INSPIRE].

[42] G. Bevilacqua, M. Czakon, A. van Hameren, C.G. Papadopoulos and M. Worek, Complete off-shell effects in top quark pair hadroproduction with leptonic decay at next-to-leading order, JHEP 02 (2011) 083 [arXiv: 1012.4230] [INSPIRE]. 
[43] A. Denner, S. Dittmaier, S. Kallweit and S. Pozzorini, NLO QCD corrections to off-shell top-antitop production with leptonic decays at hadron colliders, JHEP 10 (2012) 110 [arXiv: 1207.5018] [INSPIRE].

[44] R. Frederix, Top Quark Induced Backgrounds to Higgs Production in the $W W^{(*)} \rightarrow l l \nu \nu$ Decay Channel at Next-to-Leading-Order in QCD, Phys. Rev. Lett. 112 (2014) 082002 [arXiv: 1311.4893] [INSPIRE].

[45] F. Cascioli, S. Kallweit, P. Maierhöfer and S. Pozzorini, A unified NLO description of top-pair and associated Wt production, Eur. Phys. J. C 74 (2014) 2783 [arXiv:1312.0546] [INSPIRE].

[46] G. Heinrich, A. Maier, R. Nisius, J. Schlenk and J. Winter, NLO QCD corrections to $W^{+} W^{-} b \bar{b}$ production with leptonic decays in the light of top quark mass and asymmetry measurements, JHEP 06 (2014) 158 [arXiv: 1312.6659] [INSPIRE].

[47] A. Denner and R. Feger, NLO QCD corrections to off-shell top-antitop production with leptonic decays in association with a Higgs boson at the LHC, JHEP 11 (2015) 209 [arXiv: 1506.07448] [INSPIRE].

[48] A. Denner and M. Pellen, NLO electroweak corrections to off-shell top-antitop production with leptonic decays at the LHC, JHEP 08 (2016) 155 [arXiv:1607.05571] [INSPIRE].

[49] P. Draggiotis, R.H.P. Kleiss and C.G. Papadopoulos, On the computation of multigluon amplitudes, Phys. Lett. B 439 (1998) 157 [hep-ph/9807207] [INSPIRE].

[50] P.D. Draggiotis, R.H.P. Kleiss and C.G. Papadopoulos, Multijet production in hadron collisions, Eur. Phys. J. C 24 (2002) 447 [hep-ph/0202201] [INSPIRE].

[51] C.G. Papadopoulos and M. Worek, Multi-parton cross sections at hadron colliders, Eur. Phys. J. C 50 (2007) 843 [hep-ph/0512150] [INSPIRE].

[52] M. Czakon, C.G. Papadopoulos and M. Worek, Polarizing the Dipoles, JHEP 08 (2009) 085 [arXiv: 0905.0883] [INSPIRE].

[53] A. Kanaki and C.G. Papadopoulos, HELAC: A package to compute electroweak helicity amplitudes, Comput. Phys. Commun. 132 (2000) 306 [hep-ph/0002082] [INSPIRE].

[54] A. Cafarella, C.G. Papadopoulos and M. Worek, Helac-Phegas: A generator for all parton level processes, Comput. Phys. Commun. 180 (2009) 1941 [arXiv:0710.2427] [INSPIRE].

[55] A. van Hameren, PARNI for importance sampling and density estimation, Acta Phys. Polon. B 40 (2009) 259 [arXiv:0710.2448] [INSPIRE].

[56] A. van Hameren, Kaleu: A General-Purpose Parton-Level Phase Space Generator, arXiv: 1003.4953 [INSPIRE].

[57] P. Nogueira, Automatic Feynman graph generation, J. Comput. Phys. 105 (1993) 279 [INSPIRE].

[58] G. 't Hooft and M.J.G. Veltman, Regularization and Renormalization of Gauge Fields, Nucl. Phys. B 44 (1972) 189 [inSPIRE].

[59] J. Alwall et al., The automated computation of tree-level and next-to-leading order differential cross sections and their matching to parton shower simulations, JHEP 07 (2014) 079 [arXiv: 1405.0301] [INSPIRE].

[60] A. van Hameren, C.G. Papadopoulos and R. Pittau, Automated one-loop calculations: A proof of concept, JHEP 09 (2009) 106 [arXiv:0903.4665] [INSPIRE]. 
[61] G. Ossola, C.G. Papadopoulos and R. Pittau, CutTools: A program implementing the OPP reduction method to compute one-loop amplitudes, JHEP 03 (2008) 042 [arXiv:0711.3596] [INSPIRE].

[62] A. van Hameren, OneLOop: For the evaluation of one-loop scalar functions, Comput. Phys. Commun. 182 (2011) 2427 [arXiv: 1007.4716] [INSPIRE].

[63] G. Ossola, C.G. Papadopoulos and R. Pittau, Reducing full one-loop amplitudes to scalar integrals at the integrand level, Nucl. Phys. B 763 (2007) 147 [hep-ph/0609007] [INSPIRE].

[64] G. Ossola, C.G. Papadopoulos and R. Pittau, On the Rational Terms of the one-loop amplitudes, JHEP 05 (2008) 004 [arXiv:0802.1876] [INSPIRE].

[65] P. Mastrolia, G. Ossola, C.G. Papadopoulos and R. Pittau, Optimizing the Reduction of One-Loop Amplitudes, JHEP 06 (2008) 030 [arXiv:0803.3964] [INSPIRE].

[66] P. Draggiotis, M.V. Garzelli, C.G. Papadopoulos and R. Pittau, Feynman Rules for the Rational Part of the QCD 1-loop amplitudes, JHEP 04 (2009) 072 [arXiv:0903.0356] [INSPIRE].

[67] S. Catani and M.H. Seymour, A general algorithm for calculating jet cross-sections in NLO QCD, Nucl. Phys. B 485 (1997) 291 [Erratum ibid. B 510 (1998) 503] [hep-ph/9605323] [INSPIRE].

[68] S. Catani, S. Dittmaier, M.H. Seymour and Z. Trócsányi, The dipole formalism for next-to-leading order QCD calculations with massive partons, Nucl. Phys. B 627 (2002) 189 [hep-ph/0201036] [InSPIRE].

[69] Z. Nagy and D.E. Soper, Parton showers with quantum interference, JHEP 09 (2007) 114 [arXiv:0706.0017] [INSPIRE].

[70] Z. Nagy and D.E. Soper, A parton shower based on factorization of the quantum density matrix, JHEP 06 (2014) 097 [arXiv: 1401.6364] [INSPIRE].

[71] G. Bevilacqua, M. Czakon, M. Kubocz and M. Worek, Complete Nagy-Soper subtraction for next-to-leading order calculations in QCD, JHEP 10 (2013) 204 [arXiv:1308.5605] [INSPIRE].

[72] A. Denner, S. Dittmaier, M. Roth and D. Wackeroth, Predictions for all processes $e^{+} e^{-} \rightarrow 4$ fermions $+\gamma$, Nucl. Phys. B 560 (1999) 33 [hep-ph/9904472] [INSPIRE].

[73] A. Denner, S. Dittmaier, M. Roth and L.H. Wieders, Electroweak corrections to charged-current $e^{+} e^{-} \rightarrow 4$ fermion processes: Technical details and further results, Nucl. Phys. B 724 (2005) 247 [Erratum ibid. B 854 (2012) 504] [hep-ph/0505042] [INSPIRE].

[74] G. Bevilacqua et al., HELAC-NLO, Comput. Phys. Commun. 184 (2013) 986 [arXiv:1110.1499] [INSPIRE].

[75] G. Bevilacqua, M. Czakon, C.G. Papadopoulos, R. Pittau and M. Worek, Assault on the NLO Wishlist: $p p \rightarrow t \bar{t} b \bar{b}$, JHEP 09 (2009) 109 [arXiv:0907.4723] [INSPIRE].

[76] G. Bevilacqua, M. Czakon, C.G. Papadopoulos and M. Worek, Dominant QCD Backgrounds in Higgs Boson Analyses at the LHC: A Study of $p p \rightarrow t \bar{t}+2$ jets at Next-To-Leading Order, Phys. Rev. Lett. 104 (2010) 162002 [arXiv:1002.4009] [INSPIRE].

[77] G. Bevilacqua, M. Czakon, C.G. Papadopoulos and M. Worek, Hadronic top-quark pair production in association with two jets at Next-to-Leading Order QCD, Phys. Rev. D 84 (2011) 114017 [arXiv:1108.2851] [INSPIRE].

[78] G. Bevilacqua and M. Worek, Constraining BSM Physics at the LHC: Four top final states with NLO accuracy in perturbative QCD, JHEP 07 (2012) 111 [arXiv:1206.3064] [INSPIRE]. 
[79] G. Bevilacqua, M. Czakon, M. Krämer, M. Kubocz and M. Worek, Quantifying quark mass effects at the LHC: A study of $p p \rightarrow b \bar{b} b \bar{b}+X$ at next-to-leading order, JHEP 07 (2013) 095 [arXiv: 1304.6860] [INSPIRE].

[80] G. Bevilacqua and M. Worek, On the ratio of $t \bar{t} b \bar{b}$ and $t \bar{t} j j$ cross sections at the CERN Large Hadron Collider, JHEP 07 (2014) 135 [arXiv: 1403.2046] [INSPIRE].

[81] Z. Bern et al., Ntuples for NLO Events at Hadron Colliders, Comput. Phys. Commun. 185 (2014) 1443 [arXiv: 1310.7439] [INSPIRE].

[82] Particle Data Group collaboration, K.A. Olive et al., Review of Particle Physics, Chin. Phys. C 38 (2014) 090001 [INSPIRE].

[83] M. Jezabek and J.H. Kuhn, QCD Corrections to Semileptonic Decays of Heavy Quarks, Nucl. Phys. B 314 (1989) 1 [inSPIRE].

[84] J. Butterworth et al., PDF4LHC recommendations for LHC Run II, J. Phys. G 43 (2016) 023001 [arXiv: 1510.03865] [INSPIRE].

[85] S. Dulat et al., New parton distribution functions from a global analysis of quantum chromodynamics, Phys. Rev. D 93 (2016) 033006 [arXiv:1506.07443] [INSPIRE].

[86] L.A. Harland-Lang, A.D. Martin, P. Motylinski and R.S. Thorne, Parton distributions in the LHC era: MMHT 2014 PDFs, Eur. Phys. J. C 75 (2015) 204 [arXiv:1412.3989] [InSPIRE].

[87] NNPDF collaboration, R.D. Ball et al., Parton distributions for the LHC Run II, JHEP 04 (2015) 040 [arXiv: 1410.8849] [INSPIRE].

[88] A. Buckley et al., LHAPDF6: parton density access in the LHC precision era, Eur. Phys. J. C 75 (2015) 132 [arXiv:1412.7420] [INSPIRE].

[89] M. Cacciari, G.P. Salam and G. Soyez, The anti-k(t) jet clustering algorithm, JHEP 04 (2008) 063 [arXiv:0802.1189] [INSPIRE].

[90] T. Plehn, M. Spannowsky and M. Takeuchi, Stop searches in 2012, JHEP 08 (2012) 091 [arXiv: 1205.2696] [INSPIRE].

[91] J. Cao, C. Han, L. Wu, J.M. Yang and Y. Zhang, Probing Natural SUSY from Stop Pair Production at the LHC, JHEP 11 (2012) 039 [arXiv:1206.3865] [INSPIRE].

[92] C.-Y. Chen, A. Freitas, T. Han and K.S.M. Lee, New Physics from the Top at the LHC, JHEP 11 (2012) 124 [arXiv: 1207.4794] [INSPIRE].

[93] R. Boughezal and M. Schulze, $t \bar{t}+$ large missing energy from top-quark partners: $A$ comprehensive study at next-to-leading order QCD, Phys. Rev. D 88 (2013) 114002 [arXiv: 1309.2316] [INSPIRE].

[94] W. Bernreuther and Z.-G. Si, Distributions and correlations for top quark pair production and decay at the Tevatron and LHC., Nucl. Phys. B 837 (2010) 90 [arXiv:1003.3926] [InSPIRE].

[95] W. Bernreuther, P. Galler, C. Mellein, Z.G. Si and P. Uwer, Production of heavy Higgs bosons and decay into top quarks at the LHC, Phys. Rev. D 93 (2016) 034032 [arXiv: 1511.05584] [INSPIRE].

[96] M. Beneke et al., Top quark physics, hep-ph/0003033 [INSPIRE]. 